\title{
Minimax Critical Points in Ginzburg-Landau Problems with Semi-stiff Boundary Conditions: Existence and Bubbling
}

\author{
Leonid Berlyand * $\quad$ Petru Mironescu ${ }^{\dagger} \quad$ Volodymyr Rybalko ${ }^{\ddagger}$ \\ Etienne Sandier ${ }^{\S}$
}

August 26, 2013

\begin{abstract}
Let $\Omega \subset \mathbb{R}^{2}$ be a smooth bounded simply connected domain. We consider the simplified GinzburgLandau energy $E_{\varepsilon}(u)=\frac{1}{2} \int_{\Omega}|\nabla u|^{2}+\frac{1}{4 \varepsilon^{2}} \int_{\Omega}\left(1-|u|^{2}\right)^{2}$, where $u: \Omega \rightarrow \mathbb{C}$. We prescribe $|u|=1$ and $\operatorname{deg}(u, \partial \Omega)=1$. In this setting, there are no minimizers of $E_{\varepsilon}$. Using a mountain pass approach, we obtain existence of critical points of $E_{\varepsilon}$ for large $\varepsilon$. Our analysis relies on Wente estimates and on the study of bubbling phenomena for Palais-Smale sequences.
\end{abstract}

\section{Introduction}

We consider a smooth bounded simply connected domain $\Omega \subset \mathbb{R}^{2}$ and set $\Gamma=\partial \Omega$. Let

$$
\mathscr{E}=\left\{u \in H^{1}(\Omega ; \mathbb{C}) ;|\operatorname{tr} u|=1\right\} .
$$

Here, $\operatorname{tr} u$ denotes the trace of $u$ on $\Gamma$. If $u \in \mathscr{E}$ and we let $g=\operatorname{tr} u$, then $g \in H^{1 / 2}\left(\Gamma ; \mathbb{S}^{1}\right)$, and therefore we may define the winding number (degree) of $g$ [15, Appendix], denoted by $\operatorname{deg}(u, \Gamma)$ or $\operatorname{deg}(g, \Gamma){ }^{1}$ In particular, for $d \in \mathbb{N}^{*}$ we may define the class

$$
\mathscr{E}_{d}=\left\{u \in H^{1}(\Omega ; \mathbb{C}) ;|\operatorname{tr} u|=1 \text { on } \Gamma, \operatorname{deg}(u, \Gamma)=d\right\} .
$$

For $\varepsilon \in(0, \infty]$, we consider the simplified Ginzburg-Landau energy

$$
E_{\varepsilon}(u)=\frac{1}{2} \int_{\Omega}|\nabla u|^{2}+\frac{1}{4 \varepsilon^{2}} \int_{\Omega}\left(1-|u|^{2}\right)^{2} .
$$

${ }^{*}$ L. B.: Department of Mathematics, The Pennsylvania State University, University Park, PA 16802, USA. Email address: berlyand@math.psu.edu

${ }^{\dagger}$ P. M.: Université de Lyon; Université Lyon 1; CNRS, UMR5208, Institut Camille Jordan, 43 blvd du 11 novembre 1918, F-69622 Villeurbanne-Cedex, France. Email address: mironescu@math.univ-lyon1.fr

${ }^{\ddagger}$ V. R.: Mathematical Division, B. Verkin Institute for Low Temperature Physics and Engineering of the National Academy of Sciences of Ukraine, 47 Lenin Ave., 61103 Kharkiv, Ukraine. Email address: vrybalko@ilt.kharkov.ua

${ }^{\S}$ E. S.: Université Paris-Est, LAMA (UMR 8050), UPEC, UPEMLV, F-94010, Créteil, France. Email address: sandier@u-pec.fr

${ }^{1}$ We will come back to the existence and the basic properties of the degree in Section 2; see in particular formula (2.4) and the explanations preceding it. In the meantime, it may be convenient to imagine that all maps are smooth and then the degree is simply the winding number of the smooth map $g: \mathbb{S}^{1} \rightarrow \mathbb{S}^{1}$. 
Our paper is devoted to the existence of critical points of $E_{\varepsilon}$ in $\mathscr{E}_{d}$, with special focus on the case $d=1$.

Boundary condition $|\operatorname{tr} u|=1$ can be regarded as a relaxation of the $\mathbb{S}^{1}$-valued Dirichlet boundary condition. This latter condition was considered in detail in the classical work [11], where the asymptotic behavior of critical points of $E_{\varepsilon}$ was studied in the limit $\varepsilon \rightarrow 0$. It was shown, in particular, that zeroes (vortices) of critical points are distant from the boundary, i.e., boundary "repels" vortices. The Ginzburg-Landau equation with the Neumann boundary condition presents another extremal effect "vortices flow through the boundary outside the domain", more precisely, there are no stable (global) minimizers with vortices [40]. The semi-stiff boundary conditions were introduced and studied in [10], [6], [9]. These conditions are intermediate between the Dirichlet and Neumann conditions in the following sense: the Dirichlet condition $|u|=1$ is assumed for the modulus and the Neumann one for the phase of $u$ on the boundary.

In order to obtain nontrivial (nonconstant) critical points one can prescribe nonzero degree on the boundary. Note that the topological degree is continuous with respect to the strong convergence in $H^{1}(\Omega ; \mathbb{C})$ and one can show that the sets $\mathscr{E}_{d}$ are the connected components of $\mathscr{E}$ (in the topology inherited from $H^{1}(\Omega ; \mathbb{C})$ ). Thus minimizers of $E_{\varepsilon}$ in $\mathscr{E}_{d}$ (if they exist) are local minimizers in $\mathscr{E}$.

A first natural issue is existence of minimizers of $E_{\varepsilon}$ in $\mathscr{E}_{d}$. When $\varepsilon=\infty$, it is easy to see that the minimizers of $E_{\infty}$ in $\mathscr{E}_{1}$ are precisely the conformal representations of $\Omega$ into the unit disc $\mathbb{D}$; it is also possible to characterize the minimizers $E_{\infty}$ in $\mathscr{E}_{d}$ for $d \geq 2$ (Corollary 3.2). When $\varepsilon<\infty, E_{\varepsilon}$ does not attain its minimum in $\mathscr{E}_{d}$ unless $d=0$ (Lemma 3.4). This contrasts with the case of thin doubly connected domains, in which minimizers of $E_{\varepsilon}$ with prescribed degrees one and one do exist [28], [6]. ${ }^{2}$

Next natural question is existence of critical points. Our main result is the following.

\subsection{Theorem. There exists some $\varepsilon_{0}>0$ such that, for $\varepsilon>\varepsilon_{0}, E_{\varepsilon}$ has critical points in $\mathscr{E}_{1}$.}

The interesting features of existence/nonexistence of critical points were observed in [10]. Next a nontrivial result on the existence of critical points for the Ginzburg-Landau functional with semi-stiff boundary conditions was obtained in [28] for annuli by minimization of $E_{\varepsilon}$ with prescribed degrees $d$ on both connected component of the boundary. This result (in the case $d=1$ ) was improved and extended in [6] to general doubly connected domains. The existence/nonexistence study for doubly connected domains was completed in [4]. Works [6] and [4] show that the existence of minimizers crucially depends on the upper energy bound obtained by minimizing the Dirichlet energy among $\mathbb{S}^{1}$-valued maps. ${ }^{3}$ Namely, if this bound does not exceed a certain threshold then minimizers exist for all $\varepsilon$, otherwise they exist for large $\varepsilon$ and do not exist for small $\varepsilon$. It can be conjectured on the basis of aforementioned works that global minimizers with prescribed degrees either do not exist or, if they exist, have no zeroes (vortices). However, in [9] it was shown that critical points with zeroes (vortices) do exist (for small $\varepsilon$ ) for all prescribed degrees on components of a doubly connected domain. The method in [9] makes use of nontrivial topological structure of energy sublevel sets in the case of doubly connected domain, and critical points found in [9] are local minimizers. This approach extends to general multiply connected domains [23], but cannot be applied to find critical points in simply connected domains. An important tool in the construction of [9] is the approximate bulk degree functional, introduced in that work for doubly connected domains. The latter notion can be generalized to multiply connected domains [23]; however, it does not have an analogue for simply connected domains.

The techniques developed in the works cited above for semi-stiff boundary conditions do not lead to (locally or globally) minimizing solutions in simply connected domains. Thus a natural question

\footnotetext{
${ }^{2} \mathrm{~A}$ well-known similar situation occurs for the equation $-\Delta u=u^{(n+2) /(n-2)}$ in $\Omega \subset \mathbb{R}^{n}$ with $n \geq 3$, where a non trivial topology leads to the existence of critical points [22], [3].

${ }^{3}$ In turn, this bound can be explicitly expressed in terms of the $H^{1}$-capacity of the domain.
} 
arises: can one find minimax (saddle) critical points for semi-stiff boundary conditions? This question motivated the present work.

By contrast with the above references and especially [9], our approach is not based on the direct method: in Theorem 1.1, critical points are minimax type ones; their existence is obtained via the Mountain Pass Theorem of Ambrosetti and Rabinowitz [1] combined with an asymptotic analysis of the Palais-Smale sequences. This analysis is rather delicate, since our problem is non compact. Non compact problems have been broadly considered in the PDE literature for more then three decades. They include nonlinear problems with critical growth (in particular, the celebrated Yamabe problem), three body problem, Yang-Mills equations, harmonic map problem etc. (see e.g. the references in [16]). In non compact problems, analysis of Palais-Smale sequences and the validity of the $(P S)_{c}$ (Palais-Smale condition at the energy level $c$ ) condition of Brezis, Coron and Nirenberg [18] play a crucial role. This analysis is also at the heart of the proof of Theorem 1.1.

After our work was completed, our analysis for large $\varepsilon$ was complemented in [32] by several existence results for small $\varepsilon$. The main results in [32] assert that, for small $\varepsilon, E_{\varepsilon}$ has critical points with prescribed degree one provided:

1. Either $\Omega$ is close (in a suitable sense) to a disc.

2. Or $\Omega$ is a generic (in a suitable sense) simply connected domain.

Unlike the methods we develop here, the approach in [32] relies on inverse function techniques, in the spirit of the construction of critical points of $E_{\varepsilon}$ with Dirichlet boundary condition performed by Pacard and Rivière [36]. However, the two approaches have something in common: they are restricted to the prescribed degree one. The proof in the present paper is based on a contradiction argument which works only for relatively small energies (less than $2 \pi$ ); this is why we consider here only the case of degree one. More specifically, the argument goes as follows. We first establish existence of a mountain pass geometry for the energy functional $E_{\varepsilon}$ for an appropriate choice of the function space. Using the Mountain Pass Theorem [1], this leads to the existence of sequences of almost critical points of $E_{\varepsilon}$. The key point is then to prove the following compactness result: any possible weak limit $u$ of such a sequence still has degree one. ${ }^{4}$ The above compactness is obtained by contradiction. To be more precise, we prove that, up to a subsequence, a non compact sequence $\left(u_{n}\right)$ has exactly one vortex approaching the boundary and that the energy $E_{\varepsilon}\left(u_{n}\right)$ asymptotically splits into the sum of two terms: the first one equals $\pi$ and corresponds to the energy concentrated near the vortex, while the second term is $E_{\varepsilon}(u)$ (the energy of the weak limit $u$ of the sequence). When the energies of the $u_{n}$ 's are asymptotically below the critical value $2 \pi$, the weak limit $u$ turns out to be a critical point of $E_{\varepsilon}$ whose energy lies in the interval $(0, \pi)$. We complete the proof by proving that there is no such a critical point minimizing the functional with respect to its own boundary conditions.

This approach however cannot be directly applied for finding critical points with degrees greater than one; this remains an interesting open problem.

Our paper is organized as follows. In Section 2, we recall the existence and the basic properties of the boundary degree for maps in $\mathscr{E}$, as well as some applications of the Wente estimates, which are crucial in the analysis of the Palais-Smale sequences. In Section 3, we prove the (non)existence of minimizers of $E_{\varepsilon}$. The basic objects involved in this analysis are the Blaschke products, that play a crucial role in what follows. Section 4 gives the structure of maps close (in a suitable sense) to Moebius transforms, and more generally to Blaschke products. The results in Section 4 are used in Section 5 for establishing a mountain pass geometry (in a suitable functional setting). In Section 5 , we rely on the Mountain Pass Theorem in order to obtain sequences of almost critical points of $E_{\varepsilon}$ and an almost critical level $c>\pi$. In Section 6 we prove Theorem 1.1. The rather simple proof requires

\footnotetext{
${ }^{4}$ Such a limit is a critical point of $E_{\varepsilon}$ with degree one.
} 
both $\varepsilon$ large and $c<2 \pi$. Once the existence of critical points is established, we determine in Section 7 their behavior as $\varepsilon \rightarrow \infty$. We next turn to the task of generalizing Theorem 1.1 to a larger range of $\varepsilon$ 's. This relies on a careful analysis of the Palais-Smale sequences. Although Theorem 1.1 is about degree 1 and simply connected domains, we found interesting to present in Sections 8 and 9 the analysis of the Palais-Smale sequences when the boundary degree is arbitrary and the domain is allowed to be multiply connected. ${ }^{5}$ We next rely on this analysis in order to obtain a slight generalization of Theorem 1.1; see Theorem 8.14. In Section 9, we present an application of the bubbling analysis in multiply connected domains. More specifically we obtain, in thick circular annuli and for large $\varepsilon$, existence of critical points with prescribed degrees one and zero (Theorem 9.6).

Acknowledgements. We are grateful to J.-M.Coron for a very helpful conversation in the early stages of this paper. The work of LB was supported by NSF grant DMS-1106666. The work of VR was partially supported by NSF grant DMS-1106666. PM was supported by the ANR project ANR12-BS01-0013-03.

\section{Contents}

1 Introduction $\quad 1$

2 Functional setting $\quad 4$

3 The basic example $\quad 15$

4 Moebius and almost Moebius transforms $\quad 17$

5 Mountain pass approach $\quad 23$

6 Existence of critical points $\quad 30$

7 Asymptotic behavior of critical points as $\varepsilon \rightarrow \infty \quad 34$

8 Bubbling analysis for small $\varepsilon \quad 40$

9 Bubbling analysis in multiply connected domains $\quad 47$

\section{Functional setting}

We start by specifying some notation used throughout the paper.

1. $\mathbb{D}$, respectively $\mathbb{S}^{1}$, denote the unit disc, respectively the unit circle. More generally, we will denote $\{z \in \mathbb{C} ;|z|<r\}$, respectively $\{z \in \mathbb{C} ;|z|=r\}$, by $\mathbb{D}_{r}$, respectively $C_{r}$.

2. $\omega$ and $\Omega$ will usually denote smooth open subsets of $\mathbb{R}^{2}$.

3. When $\omega \subset \mathbb{R}^{2}$ is smooth, $\frac{\partial}{\partial \tau}$ stands for the tangential differentiation on $\partial \omega$ endowed with its natural orientation (counterclockwise on the outer boundary of $\omega$, clockwise on the inner components of $\partial \omega)$. The notation $\frac{\partial}{\partial v}$ stands for the differentiation with respect to the outward normal.

\footnotetext{
${ }^{5}$ If we specialize to simply connected domains, some of our arguments can be substantially simplified. See e.g. Lemma 2.24 and Remark 2.25.
} 
4. If $\omega \subset \mathbb{R}^{2}$ is a smooth bounded open set and if $g \in L^{1}(\partial \omega ; \mathbb{C})$, then we let $u(g)$ denote the harmonic extension of $g$, i.e., $u(g) \in W^{1,1}(\omega)$ satisfies $\left\{\begin{array}{ll}\Delta u(g)=0 & \text { in } \omega \\ \operatorname{tr} u(g)=0 & \text { on } \partial \omega\end{array}\right.$. If in addition $g \in H^{1 / 2}(\partial \omega ; \mathbb{C})$, then we define a semi-norm in $H^{1 / 2}$ via the formula

$$
|g|_{H^{1 / 2}}^{2}=\frac{1}{2} \int_{\omega}|\nabla u(g)|^{2} .
$$

As well-known, this semi-norm is equivalent to the standard (Gagliardo) semi-norm

$$
g \mapsto\left(\int_{\partial \omega} \int_{\partial \omega} \frac{|g(x)-g(y)|^{2}}{|x-y|^{2}} d s_{x} d s_{y}\right)^{1 / 2}, \quad g \in H^{1 / 2}(\partial \omega ; \mathbb{C})^{6}
$$

5. $\wedge$ stands for the vector product of complex numbers: $\left(a_{1}+\imath a_{2}\right) \wedge\left(b_{1}+\imath b_{2}\right)=a_{1} b_{2}-a_{2} b_{1}$. Similarly, the notation $u \wedge \nabla v$, with $u$ and $v$ complex-valued functions, stands for the vector-field $u_{1} \nabla v_{2}-$ $u_{2} \nabla v_{1}$.

6. If $u \in C^{1}(\omega ; \mathbb{C}), u=u_{1}+\imath u_{2}$, then Jac $u=\nabla u_{1} \wedge \nabla u_{2}=\partial_{x} u \wedge \partial_{y} u$ is the Jacobian determinant of $u$. When $\omega$ is smooth bounded and $u \in C^{1}(\bar{\omega})$, we note the integration by parts formula

$$
\int_{\omega} \operatorname{Jac} u=\frac{1}{2} \int_{\partial \omega} u \wedge \frac{\partial u}{\partial \tau} .
$$

Formula (2.2) extends to $H^{1}$ maps; see Lemma 2.3.

7. If $(a, b) \in \mathbb{R}^{2}$, then $(a, b)^{\perp}=(-b, a)$. With complex notation, $z^{\perp}=\imath z$. In the same vein, for functions $f=f(x, y)$, we let $\nabla^{\perp} f=\left(-\partial_{y} f, \partial_{x} f\right)$.

8. With the above notation, we have $\operatorname{Jac}(f, g)=-\nabla f \cdot \nabla^{\perp} g$.

9. - stands for the real scalar product. E.g., we have $\left(a_{1}+\imath a_{2}\right) \cdot\left(b_{1}+\imath b_{2}\right)=a_{1} b_{1}+a_{2} b_{2}$, and if $u=u_{1}+\imath u_{2}$ and $v=v_{1}+\imath v_{2}$ are complex vectors, then $u \cdot v=u_{1} \cdot v_{1}+u_{2} \cdot v_{2}$.

10. Several function spaces will appear frequently:

a) $\mathscr{E}=\left\{u \in H^{1}(\Omega ; \mathbb{C}) ;|\operatorname{tr} u|=1\right\}$. If, in addition, $\Omega$ is simply connected and $d \in \mathbb{Z}$, then $\mathscr{E}_{d}=\{u \in$ $\mathscr{E} ; \operatorname{deg}(u, \partial \Omega)=d\}$.

b) When $\Omega=\mathbb{D}$, the two above spaces are denoted $\mathscr{G}$, respectively $\mathscr{G}_{d}$.

c) We let $\mathscr{H}=H^{1 / 2}\left(\mathbb{S}^{1} ; \mathbb{S}^{1}\right)$ and $\mathscr{H}_{d}=\{g \in \mathscr{H} ; \operatorname{deg} g=d\}$.

We next turn to the description of a functional setting adapted to the study of critical points.

If $g \in H^{1 / 2}\left(\mathbb{S}^{1} ; \mathbb{C}\right)$, then the semi-norm $|g|_{H^{1 / 2}}$ is easily expressed in terms of Fourier coefficients: if we write $g=\sum a_{n} e^{\imath n \theta}$, then

$$
|g|_{H^{1 / 2}}^{2}=\pi \sum|n|\left|a_{n}\right|^{2}
$$

As noticed first by Boutet de Monvel and Gabber [15, Appendix], a map $g \in H^{1 / 2}\left(\mathbb{S}^{1} ; \mathbb{S}^{1}\right)$ has a well-defined winding number (degree), denoted deg $\left(g, \mathbb{S}^{1}\right)$, or simply deg $g$. This degree is defined as

\footnotetext{
${ }^{6}$ When $\omega$ is simply connected, this equivalence is already implicit in Gagliardo's paper on trace theory [25].
} 
follows. On the one hand, $C^{\infty}\left(\mathbb{S}^{1} ; \mathbb{S}^{1}\right)$ is dense in $H^{1 / 2}\left(\mathbb{S}^{1} ; \mathbb{S}^{1}\right)$ [15, Appendix]. ${ }^{7}$ On the other hand, if we write in Fourier series a smooth circle-valued map $g$ as $g=\sum a_{n} e^{\imath n \theta}$, then

$$
\operatorname{deg} g=\sum n\left|a_{n}\right|^{2} \text {. }
$$

Via (2.3), we easily obtain that the degree of smooth maps is continuous with respect to the $H^{1 / 2}$ convergence. This implies that the right side of (2.4) is an integer for each map $g \in H^{1 / 2}\left(\mathbb{S}^{1} ; \mathbb{S}^{1}\right)$; this integer is the degree of $g$.

When $\mathbb{S}^{1}$ is replaced by a smooth simple closed planar curve $\Gamma$, the degree of a map $g \in H^{1 / 2}\left(\Gamma ; \mathbb{S}^{1}\right)$ can be defined using the above procedure: we first establish density of $C^{\infty}\left(\Gamma ; \mathbb{S}^{1}\right)$ into $H^{1 / 2}\left(\Gamma ; \mathbb{S}^{1}\right)$, next prove continuity of the degree of smooth maps for $H^{1 / 2}$ convergence. An alternative equivalent approach is the following. Let $\Omega$ be the domain enclosed by the curve $\Gamma$. We fix some conformal representation $\Phi \in C^{\infty}(\bar{\Omega} ; \overline{\mathbb{D}})$. Let $\Psi=\Phi_{\mid \Gamma}: \Gamma \rightarrow \mathbb{S}^{1}$. If $g \in H^{1 / 2}\left(\Gamma ; \mathbb{S}^{1}\right)$, then we have $g \circ \Psi^{-1} \in H^{1 / 2}\left(\mathbb{S}^{1} ; \mathbb{S}^{1}\right)$ and thus we may set

$$
\operatorname{deg}(g, \Gamma)=\operatorname{deg}\left(g \circ \Psi^{-1}, \mathbb{S}^{1}\right) .
$$

Formula (2.5) reduces the study of degree to the case where $\Gamma=\mathbb{S}^{1}$ and $\Omega=\mathbb{D}$. Existence of the degree suffices to describe the functional setting we need. However, in subsequent sections we will use further properties of the degree. For the convenience of the reader, these properties are recalled below. The results we present are well-known to the experts but difficult to find in the literature. We follow mainly unpublished lecture notes of a graduate course of $\mathrm{H}$. Brezis at Paris 6 . Some of the results in this section are also presented in [15, 20, 21, 14, 19]. Proofs of Lemmas 2.1-2.3 and 2.5-2.8 are part of Ginzburg-Landau folklore, but most of them unavailable; see however [37, p. 37-41] for some proofs.

2.1 Lemma. 1. $C^{\infty}\left(\Gamma ; \mathbb{S}^{1}\right)$ is dense in $H^{1 / 2}\left(\Gamma ; \mathbb{S}^{1}\right)$.

2. The degree of $H^{1 / 2}\left(\Gamma ; \mathbb{S}^{1}\right)$ maps is continuous with respect to the strong $H^{1 / 2}$-convergence.

3. The degree of $H^{1 / 2}\left(\Gamma ; \mathbb{S}^{1}\right)$ maps is not continuous with respect to the weak $H^{1 / 2}$-convergence.

Proof. The first two items rephrase the beginning of this section. For the third one, we let $\Gamma=\mathbb{S}^{1}$ and proceed as follows: let $g_{a}(z)=\frac{z-a}{1-a z}, z \in \mathbb{S}^{1}, a \in(0,1)$. Then $g_{a} \rightarrow-1$ as $a \rightarrow 1$ and $\operatorname{deg} g_{a}=1$, but $\operatorname{deg}(-1)=0$.

The lack of continuity of the degree with respect to weak convergence makes the minimization of $E_{\varepsilon}$ in $\mathscr{E}_{d}$ non trivial.

We continue with a result relating the degree of $H^{1 / 2}$ maps to the more familiar degree of continuous maps.

2.2 Lemma. Let $u \in H^{1}(\mathbb{D} ; \mathbb{C}) \cap C(\mathbb{D} ; \mathbb{C})$. Assume that $0<c \leq|u| \leq C<\infty$ in a neighborhood of $\mathbb{S}^{1}$ and that $|\operatorname{tr} u|=1$. Then, for $r$ close to 1 , we have

$$
\operatorname{deg}\left(\operatorname{tr} u, \mathbb{S}^{1}\right)=\operatorname{deg}\left(u, C_{r}\right)=\operatorname{deg}\left(u /|u|, C_{r}\right) .
$$

In the remaining part of this section, unless otherwise stated, $\Omega$ is a smooth bounded simply connected domain and $\Gamma=\partial \Omega$.

We continue with two useful formulas giving the degree. The first one is merely an interpretation of (2.4).

\footnotetext{
${ }^{7}$ The point here is not density of smooth maps, but density of smooth circle-valued maps.
} 
2.3 Lemma. Let $g \in H^{1 / 2}\left(\Gamma ; \mathbb{S}^{1}\right)$ and let $u \in H^{1}(\Omega ; \mathbb{C})$ be such that $\operatorname{tr} u=g$. Then

$$
\operatorname{deg} g=\frac{1}{2 \imath \pi}\left\langle\frac{\partial g}{\partial \tau}, g\right\rangle_{H^{-1 / 2}, H^{1 / 2}}=\frac{1}{2 \pi} \int_{\Gamma} g \wedge \frac{\partial g}{\partial \tau}
$$

and

$$
\operatorname{deg} g=\frac{1}{\pi} \int_{\Omega} \operatorname{Jac} u
$$

The second equality in (2.7) is valid only when $g$ is sufficiently smooth, say $g \in C^{1}$. In this paper, we often use this equality, but the integral has to be understood in the sense of the $H^{1 / 2}-H^{-1 / 2}$ duality.

2.4 Corollary. Let $u \in H^{1}(\Omega ; \mathbb{C})$.

1. If $|u|=1$ on $\partial \Omega$, then

$$
\int_{\Omega}|\nabla u|^{2} \geq 2 \pi|\operatorname{deg}(\operatorname{tr} u, \partial \Omega)|
$$

2. If $|u| \geq \rho>0$ on $\partial \Omega$, then

$$
\int_{\Omega}|\nabla u|^{2} \geq 2 \pi \rho^{2}|\operatorname{deg}(\operatorname{tr} u, \partial \Omega)| .
$$

Proof. The first conclusion above is obtained by combining (2.8) with the pointwise inequality $2|\operatorname{Jac} u| \leq$ $|\nabla u|^{2}$. The second one is obtained by applying (2.9) to $\Phi(u)$, where $\Phi(z)=\left\{\begin{array}{ll}z / \rho, & \text { if }|z| \leq \rho \\ z /|z|, & \text { if }|z|>\rho\end{array}\right.$.

As one may expect, the degree of $H^{1 / 2}$ maps inherits some well-known properties of the degree of continuous maps.

2.5 Lemma. deg is locally constant in $H^{1 / 2}\left(\Gamma ; \mathbb{S}^{1}\right)$.

2.6 Lemma. Let $g, h \in H^{1 / 2}\left(\Gamma ; \mathbb{S}^{1}\right)$. Then the following hold.

1. If $g$ is continuous, then the degree of $g$ in the sense $H^{1 / 2}$ maps is the same as the degree of $g$ in the sense of continuous maps.

2. $\operatorname{deg}(g h)=\operatorname{deg} g+\operatorname{deg} h$.

3. $\operatorname{deg}(g / h)=\operatorname{deg} g-\operatorname{deg} h$.

4. $\operatorname{deg} g=0 \Longleftrightarrow g=e^{\imath \psi}$ for some $\psi \in H^{1 / 2}(\Gamma ; \mathbb{R})$.

More generally, deg $g=d \Longleftrightarrow g=g_{0} e^{\imath \psi}$ for some $\psi \in H^{1 / 2}(\Gamma ; \mathbb{R})$ and a fixed smooth reference map $g_{0} \in C^{\infty}\left(\Gamma ; \mathbb{S}^{1}\right)$ of degree $d$.

Item 4. above gives a first characterization of maps with zero degree. Further characterization are related to the existence of circle-valued extensions. 
2.7 Lemma. 1. Let $u \in H^{1}(\Omega ; \mathbb{C})$ be such that essinf $|u|>0$. Then we may write $u=\rho e^{i \varphi}$, where $\rho=|u| \in H^{1}(\Omega ; \mathbb{R})$ and $\varphi \in H^{1}(\Omega ; \mathbb{R})$.

2. Let $g \in H^{1 / 2}\left(\Gamma ; \mathbb{S}^{1}\right)$. Then

$$
\begin{aligned}
\operatorname{deg} g=0 & \Longleftrightarrow g=\operatorname{tr} u \text { for some } u \in H^{1}\left(\Omega ; \mathbb{S}^{1}\right) \\
& \Longleftrightarrow g=\operatorname{tr} u \text { for some } u \in H^{1}(\Omega ; \mathbb{C}) \text { such that essinf }|u|>0 .
\end{aligned}
$$

3. Let $g \in H^{1 / 2}\left(\Gamma ; \mathbb{S}^{1}\right)$ be such that $\operatorname{deg} g=0$. Write $g=e^{\imath \psi}$, with $\psi \in H^{1 / 2}(\Gamma ; \mathbb{R})$ and define $H_{g}^{1}\left(\Omega ; \mathbb{S}^{1}\right):=$ $\left\{u \in H^{1}(\Omega ; \mathbb{C}) ;|u|=1\right.$ and $\left.\operatorname{tr} u=g\right\}$. Then we have

$$
H_{g}^{1}\left(\Omega ; \mathbb{S}^{1}\right)=\left\{e^{l \varphi} ; \varphi \in H^{1}(\Omega ; \mathbb{R}) \text { and } \operatorname{tr} \varphi=\psi\right\} .
$$

Similarly, for $C>0$ we have the equality

$$
\left\{u \in H^{1}(\Omega ; \mathbb{C}) ; \operatorname{essinf}|u| \geq C, \operatorname{tr} u=g\right\}=\left\{\rho e^{\imath \varphi} ; \rho, \varphi \in H^{1}(\Omega ; \mathbb{R}), \operatorname{tr} \varphi=\psi, \operatorname{essinf} \rho \geq C\right\} .
$$

Let us also note that, if we may write $u=\rho e^{\imath \varphi}$ for some $\varphi \in H^{1}$ and for some $\rho \in H^{1} \cap L^{\infty}$ such that essinf $\rho>0$, then we have the identities

$$
|\nabla u|^{2}=|\nabla \rho|^{2}+\rho^{2}|\nabla \varphi|^{2} \quad \text { and } \quad \nabla \varphi=\left(\frac{u}{\rho}\right) \wedge \nabla\left(\frac{u}{\rho}\right)=\frac{1}{\rho^{2}} u \wedge \nabla u .
$$

Like for continuous maps, in a multiply connected domain $\omega$ a circle-valued $H^{1}$ map $u$ need not have a global phase $\varphi$ as in Lemma 2.7. However, as a consequence of Lemma 2.71 , if essinf $|u|>0$ then we may locally write

$$
u=\rho e^{\imath \varphi} \text {, with } \rho=|u| \text { and such that (2.11) holds. }
$$

In particular, $\nabla \varphi$ is globally defined. Global existence of $\varphi$ itself is governed by the next result, which is the $H^{1}$-counterpart of a well-known property of continuous circle-valued maps.

2.8 Lemma. Let $\omega \subset \mathbb{R}^{2}$ be a smooth bounded domain. Let $u \in H^{1}(\omega ; \mathbb{C})$ be such that essinf $|u|>0$. Then

$$
u=|u| e^{\imath \varphi}, \text { with } \varphi \in H^{1}(\omega ; \mathbb{R}) \Longleftrightarrow \operatorname{deg}\left(\frac{u}{|u|}, \gamma\right)=0, \forall \gamma \text { component of } \partial \omega .
$$

2.9 Corollary. Let $\omega \subset \mathbb{R}^{2}$ be a smooth bounded domain. Let $u_{j} \in H^{1}(\omega ; \mathbb{C}), j \in \llbracket 1,2 \rrbracket$, be such that $0<$ essinf $\left|u_{j}\right| \leq \operatorname{esssup}\left|u_{j}\right|<\infty, j \in \llbracket 1,2 \rrbracket$. Assume that $\operatorname{deg}\left(\frac{u_{1}}{\left|u_{1}\right|}, \gamma\right)=\operatorname{deg}\left(\frac{u_{2}}{\left|u_{2}\right|}, \gamma\right)$, for each component $\gamma$ of $\partial \omega$.

Then we may write $u_{2}=u_{1} \eta e^{\imath \varphi}$, where $\eta=\left|\frac{u_{2}}{u_{1}}\right| \in H^{1}(\omega ; \mathbb{R})$ and $\varphi \in H^{1}(\omega ; \mathbb{R})$.

By Lemma 2.1 , the class $\mathscr{E}_{d}$ (and in particular $\mathscr{G}_{d}$ ) is closed with respect to strong $H^{1}$ convergence, but is not closed with respect to the weak $H^{1}$ convergence. However, we do have weak closedness in absence of vortices, as explained in the next couple of results.

Let $\omega \subset \mathbb{R}^{2}$ be smooth and bounded. Let $\Gamma_{j}, j \in \llbracket 1, k \rrbracket$, be the components of $\partial \omega$. Consider, for $\lambda>0$, the class $\mathscr{H}^{(\lambda)}:=\left\{u \in H^{1}(\omega ; \mathbb{C}) ;|u| \geq \lambda\right\}$. For $u \in \mathscr{H}^{(\lambda)}, \operatorname{let} \mathbf{d}(u) \in \mathbb{Z}^{k}, \mathbf{d}_{j}(u):=\operatorname{deg}\left(u /|u|, \Gamma_{j}\right)$. 
2.10 Lemma. Let $\left(u_{n}\right) \subset \mathscr{H}^{(\lambda)}$ be such that $u_{n} \rightarrow u$ weakly in $H^{1}$. Then, for large $n$, we have $\mathbf{d}\left(u_{n}\right)=$ $\mathbf{d}(u)$.

In particular, for every $\mathbf{d} \in \mathbb{Z}^{k}$, the class

$$
\mathscr{H}_{\mathbf{d}}^{(\lambda)}:=\left\{u \in \mathscr{H}^{(\lambda)} ; \mathbf{d}(u)=\mathbf{d}\right\}
$$

is weakly closed.

Proof. The above lemma follows essentially from the results of White on the existence of homotopical invariants [43], but we present below a simple direct proof.

It is straightforward that, if $u_{n} \rightarrow u$, then $\frac{u_{n}}{\left|u_{n}\right|} \rightarrow \frac{u}{|u|}$. Therefore, we may assume that all the maps are circle-valued.

Let $f \in C^{\infty}(\bar{\omega})$. Then we have the identity [6, (2.2)]

$$
2 \int_{\omega} f \operatorname{Jac} u_{n}=\int_{\partial \omega} f u_{n} \wedge \frac{\partial u_{n}}{\partial \tau}+\int_{\omega}\left(\frac{\partial f}{\partial y} u_{n} \wedge \frac{\partial u_{n}}{\partial x}-\frac{\partial f}{\partial x} u_{n} \wedge \frac{\partial u_{n}}{\partial y}\right) .
$$

Since $u_{n}$ is circle-valued, we have Jac $u_{n} \equiv 0$ [19]. Combining this fact with (2.7) we find that, if $f$ is such that $f=1$ on $\Gamma_{j}$ and $f=0$ on $\partial \omega \backslash \Gamma_{j}$, then (2.13) becomes

$$
\operatorname{deg}\left(u_{n}, \Gamma_{j}\right)= \pm \frac{1}{2 \pi} \int_{\omega}\left(\frac{\partial f}{\partial y} u_{n} \wedge \frac{\partial u_{n}}{\partial x}-\frac{\partial f}{\partial x} u_{n} \wedge \frac{\partial u_{n}}{\partial y}\right)
$$

where, according to the orientation of $\Gamma_{j}$, the minus sign corresponds to the outer component of $\partial \omega$, and the plus sign corresponds to all other components of $\partial \omega$. We conclude via the fact that the righthand side of (2.14) is continuous with respect to the weak $H^{1}$ convergence of uniformly bounded maps.

By combining Lemma 2.10 with Corollary 2.9 and with (2.11), we obtain the following straightforward consequence, whose proof is left to the reader.

2.11 Lemma. Let $\omega$ be as above. Let $\left(u_{n}\right) \subset \mathscr{H}^{(\lambda)}$ satisfy $u_{n} \rightarrow u$ and $\left|u_{n}\right| \leq \Lambda$.

Then, for large $n$, we may write $u_{n}=u \eta_{n} e^{\imath \psi_{n}}$, with $\lambda / \Lambda \leq \eta_{n} \leq \Lambda / \lambda$ and $\eta_{n} \rightarrow 1, \psi_{n} \rightarrow 0$ in $H^{1}(\omega ; \mathbb{R})$.

The next result is essentially due to Brezis and Nirenberg [21, Theorem A3.2].

2.12 Lemma. Let $u \in H^{1}(\Omega ; \mathbb{C})$ satisfy $\Delta u \in L^{\infty}$ and $|\operatorname{tr} u|=1$. Then

$$
\lim _{z \rightarrow \partial \Omega}|u(z)|=1
$$

Proof. When $\Delta u=0,(2.15)$ follows by combining [21, Theorem A3.2] with the embedding $H^{1 / 2}(\Gamma) \hookrightarrow$ VMO.

In the general case, we write $u=v+w$, with $v$ harmonic and $\operatorname{tr} w=0$. Then $w \in C^{0}(\bar{\Omega})$ and thus $\lim _{z \rightarrow \partial \Omega} w(z)=0$. We conclude via the fact that $\lim _{z \rightarrow \partial \Omega}|v(z)|=1$.

We will also need the following version of Lemma 2.12 .

2.13 Lemma. Let $u_{j}, u \in H^{1}(\Omega ; \mathbb{C})$ be such that $\left|\operatorname{tr} u_{j}\right|=1, u_{j} \rightarrow u$ in $H^{1}$ and $\left|\Delta u_{j}\right| \leq C$. Then

$$
\left|u_{j}\right| \rightarrow|u| \quad \text { uniformly in } \bar{\Omega} \text {. }
$$


Proof. By standard interior estimates [27, Theorem 9.11, p. 235], we have $u_{j} \rightarrow u$ uniformly on compact subsets of $\Omega$.

In order to describe the boundary behavior of $u_{j}$, write, as in the previous lemma, $u_{j}=v_{j}+w_{j}$ and $u=v+w$. The proof of [21, Theorem A3.2] gives the following uniform convergence:

$$
\lim _{z \rightarrow \partial \Omega} \inf _{j}\left|v_{j}(z)\right|=1 \text {. }
$$

On the other hand, $w_{j} \rightarrow w$ in $W^{2, p}(\Omega), \forall p \in(1, \infty)$, by standard elliptic estimates [27, Theorem 9.15, p. 241], and thus $w_{j} \rightarrow w$ uniformly in $\bar{\Omega}$.

We conclude by combining this uniform convergence with (2.17).

We next return to our initial problem of finding critical points of $E_{\varepsilon}$ in $\mathscr{E}_{d}$ and transfer it from $\Omega$ to $\mathbb{D}$. To this end we consider a fixed conformal representation $\Phi: \Omega \rightarrow \mathbb{D}$. Let $w=\operatorname{Jac}^{-1} \in$ $C^{\infty}(\overline{\mathbb{D}} ;(0,+\infty))$ and set $\beta=\frac{1}{\varepsilon^{2}} w$. We associate to $\Phi$ and $\varepsilon$ the energy

$$
F_{\beta}(u)=\frac{1}{2} \int_{\mathbb{D}}|\nabla u|^{2}+\frac{1}{4} \int_{\mathbb{D}} \beta(x)\left(1-|u|^{2}\right)^{2}
$$

and to $\mathscr{E}$ and $\mathscr{E}_{d}$ the classes $\mathscr{G}=\left\{u \in H^{1}(\mathbb{D} ; \mathbb{C}) ;|\operatorname{tr} u|=1\right\}$, respectively

$$
\mathscr{G}_{d}=\left\{u \in H^{1}(\mathbb{D} ; \mathbb{C}) ;|\operatorname{tr} u|=1, \operatorname{deg}\left(u, \mathbb{S}^{1}\right)=d\right\} .
$$

In view of the conformal invariance of the Dirichlet integral, it is easy to see that finding critical points (or minimizers) of $E_{\varepsilon}$ in $\mathscr{E}_{d}$ is equivalent to finding critical points (or minimizers) of $F_{\beta}$ in $\mathscr{G}_{d}$. It will be convenient to consider the energy $F_{\beta}$ for more general weights. In what follows, we always assume that

$\beta$ is non negative and essentially bounded.

We continue with a brief discussion concerning the critical points of $F_{\beta}$ (with respect to the prescribed degree boundary condition, but this is implicitly assumed and will not be specified).

2.14 Definition. By a critical point of $F_{\beta}$ we mean a solution of

$$
\left\{\begin{array}{rlrl}
-\Delta u & =\beta u\left(1-|u|^{2}\right) & & \text { in } \mathbb{D} \\
|\operatorname{tr} u| & =1 & & \text { on } \mathbb{S}^{1} \\
\int_{\mathbb{D}}(u \wedge \nabla u) \cdot \nabla \zeta & =0 & & \forall \zeta \in H^{1}(\mathbb{D}) \\
\operatorname{deg}\left(u, \mathbb{S}^{1}\right) & =d & &
\end{array} .\right.
$$

The above definition is motivated by the fact that (2.21) consists of the Euler-Lagrange equations obtained by variations of $u$ with compact support in $\mathbb{D}$ and by variations of the type $u e^{\imath t f}$, where $f \in C^{\infty}(\overline{\mathbb{D}} ; \mathbb{R})$.

We warn the reader that there is another natural notion of critical point of $F_{\beta}$, when $g=\operatorname{tr} u$ is prescribed. In this case, $u$ satisfies

$$
\left\{\begin{aligned}
-\Delta u & =\beta u\left(1-|u|^{2}\right) & & \text { in } \mathbb{D} \\
u & =g & & \text { on } \mathbb{S}^{1} .
\end{aligned}\right.
$$

A solution of (2.22) will be referred to as a critical point of $F_{\beta}$ with respect to its own Dirichlet boundary condition.

Problems (2.21) and (2.22) transpose steadily to multiply connected domains. 
2.15 Lemma. Let $u$ belong to $\mathscr{G}_{d}$. Then $u$ is a critical point of $F_{\beta}$ if and only if $u \in W^{2, p}(\mathbb{D}), \forall p \in[1, \infty)$, and

$$
\left\{\begin{aligned}
-\Delta u & =\beta u\left(1-|u|^{2}\right) & & \text { in } \mathbb{D} \\
|\operatorname{tr} u| & =1 & & \text { on } \mathbb{S}^{1} \\
u \wedge \frac{\partial u}{\partial v} & =0 & & \text { on } \mathbb{S}^{1} \\
\operatorname{deg}\left(u, \mathbb{S}^{1}\right) & =d & &
\end{aligned}\right.
$$

In addition, if $\beta$ is smooth, then so is $u$.

Proof. Follow the argument in [5, Lemma 4.4].

2.16 Remark. Let us comment the condition $u \wedge \partial u / \partial v=0$ on $\mathbb{S}^{1}$. Since $u$ belongs to $W^{2, p}, p<\infty$, we have $u \in C^{1}(\overline{\mathbb{D}})$. Given some point $z \in \mathbb{S}^{1}$, we may write, locally near $z, u$ in the form $u=\rho e^{\imath \varphi}$, and then the conditions $|u|=1$ and $u \wedge \partial u / \partial v=0$ read, locally near $z$ :

$$
\rho=1 \text { and } \frac{\partial \varphi}{\partial v}=0 .
$$

We also note the that (2.24) holds for critical points of the original energy, $E_{\varepsilon}$, in $\Omega$.

For further use, let us mention the maximum principle, essentially established in [11].

2.17 Lemma. Let $u \in \mathscr{G}$ be a critical point of $F_{\beta}$ with respect to its own boundary condition $g \in$ $H^{1 / 2}\left(\mathbb{S}^{1} ; \mathbb{S}^{1}\right)$. Then $|u| \leq 1$ in $\mathbb{D}$.

In our analysis we also rely on the following Price Lemma [6, Lemma 1].

2.18 Lemma. Let $\omega \subset \mathbb{R}^{2}$ be smooth and bounded. Let $\Gamma_{j}, j \in \llbracket 1, k \rrbracket$, be the components of $\partial \Omega$. Let $\left(u_{n}\right) \subset H^{1}(\omega ; \mathbb{C})$ satisfy: $u_{n} \rightarrow u$ in $H^{1}(\omega)$, $\left|\operatorname{tr} u_{n}\right|=1, \operatorname{deg}\left(u_{n}, \Gamma_{j}\right) \equiv d_{j}$. Let $\beta \in L^{\infty}(\omega)$. Then

$$
\liminf F_{\beta}\left(u_{n}\right) \geq F_{\beta}(u)+\pi \sum_{j=1}^{k}\left|d_{j}-\operatorname{deg}\left(u, \Gamma_{j}\right)\right| .
$$

We complete this section by recalling one of the important tools in our proofs, the Wente estimates [42] in the sharp form of Bethuel and Ghidaglia [12], and some of their applications.

In the remaining part of this section, $\omega \subset \mathbb{R}^{2}$ is assumed to be smooth and bounded.

2.19 Lemma. Let $f \in H_{0}^{1}(\omega ; \mathbb{R})$ and $g, h \in H^{1}(\omega ; \mathbb{R})$. Let $u \in W_{0}^{1,1}(\omega)$ be the solution of $\Delta u=\operatorname{Jac}(g, h)$. Then:

1. We have $u \in C(\bar{\omega}) \cap H^{1}(\omega)$ and

$$
\begin{aligned}
& \|u\|_{L^{\infty}} \leq 2\|\nabla g\|_{L^{2}}\|\nabla h\|_{L^{2}}, \\
& \|\nabla u\|_{L^{2}} \leq \sqrt{2}\|\nabla g\|_{L^{2}}\|\nabla h\|_{L^{2}} .
\end{aligned}
$$

In particular, the map

$$
\left[H^{1}(\mathbb{D} ; \mathbb{R})\right]^{2} \ni(g, h) \mapsto u \in C(\bar{\omega}) \cap H^{1}(\omega)
$$

is continuous. 
2. We have

$$
\left|\int_{\omega} f \operatorname{Jac}(g, h)\right| \leq \sqrt{2}\|\nabla f\|_{L^{2}}\|\nabla g\|_{L^{2}}\|\nabla h\|_{L^{2}}
$$

\subsection{Lemma. Let}

$$
\mathbf{H}:=\left\{h \in H^{1}(\omega ; \mathbb{R}) ; \Delta h=0\right\} .
$$

Let $f \in H_{0}^{1}(\omega ; \mathbb{R}), g \in H^{1}(\omega ; \mathbb{R})$ and $h \in \mathbf{H}$. Then

$$
\left|\int_{\omega} f \nabla g \cdot \nabla h\right| \leq C(\omega)\|\nabla f\|_{L^{2}}\|\nabla g\|_{L^{2}}\|\nabla h\|_{L^{2}}
$$

Proof. We start with the simpler case where $\omega$ is simply connected. If $h \in \mathbf{H}$, let $h^{*}$ denote the harmonic conjugate of $h$, normalized by $\int_{\partial \omega} h^{*}=0$. Recall the identity

$$
\operatorname{Jac}(g, h):=-\nabla g \cdot \nabla^{\perp} h .^{8}
$$

Using (2.31) and the fact that $\nabla h^{*}=\nabla^{\perp} h$, we have $\int_{\omega} f \nabla g \cdot \nabla h=-\int_{\omega} f \operatorname{Jac}\left(g, h^{*}\right)$. By this equality and (2.28), we obtain (2.30) with $C(\omega)=\sqrt{2}$.

We next turn to a multiply connected domain. Let $\omega_{j}, j \in \llbracket 1, k \rrbracket$, be the bounded components of $\mathbb{R}^{2} \backslash \bar{\omega}$. Fix $a_{j} \in \omega_{j}$ and let $X_{j}(x):=\frac{1}{2 \pi}\left(\nabla \ln \left(x-a_{j}\right)\right)^{\perp}$. Note that each $X_{j}$ is closed. Let $X:=(\nabla h)^{\perp}$. By a standard mean value argument, we may find curves $\Gamma_{j} \subset \omega, j \in \llbracket 1, k \rrbracket$, such that the following hold:

1. $\Gamma_{j}$ is homotopic in $\bar{\omega}$ to $\partial \omega_{j}{ }^{9}$

2. $\left|\int_{\Gamma_{j}} X \cdot \tau\right| \leq C\|\nabla h\|_{L^{2}}$

Let $c_{j}:=\int_{\Gamma_{j}} X \cdot \tau$ and set $Y:=X-\sum_{j} c_{j} X_{j}$. By construction, the vector field $Y$ is closed, and satisfies $\|Y\|_{L^{2}} \leq C\|\nabla h\|_{L^{2}}$ and $\int_{\Gamma_{j}} Y \cdot \tau=0, \forall j .{ }^{10}$ Since the $\Gamma_{j}$ 's span the homotopy group $\pi_{1}(\omega)$, this implies that $\int_{\Gamma} Y \cdot \tau=0$ for each smooth closed curve $\Gamma \subset \omega$. Thus we may write $Y=\nabla u$ for some $u$, and it is easy to see that $\|\nabla u\|_{L^{2}} \leq C\|\nabla h\|_{L^{2}}$. Using (2.28), we find that

$$
\left|\int_{\omega} f \nabla g \cdot \nabla h\right|=\left|\int_{\omega} f \operatorname{Jac}(g, u)+\sum_{j} c_{j} \int_{\omega} f \nabla g \cdot X_{j}^{\perp}\right| \leq C(\omega)\|\nabla f\|_{L^{2}}\|\nabla g\|_{L^{2}}\|\nabla h\|_{L^{2}} .
$$

Recall that, given a function $f$ on $\partial \omega$, we let $u(f)$ denote the harmonic extension of $f$.

\footnotetext{
${ }^{8}$ Recall that $(a, b)^{\perp}=(-b, a)$.

${ }^{9}$ In particular, these curves span the homotopy group $\pi_{1}(\omega)$.

${ }^{10}$ The latter property follows from the identity $\int_{\Gamma_{j}} X_{l}=\left\{\begin{array}{ll}1, & \text { if } j=l \\ 0, & \text { if } j \neq l\end{array}\right.$.
} 
2.21 Lemma. If $f, g \in H^{1 / 2}(\partial \omega)$, then $u:=u(f g)-u(f) u(g)$ belongs to $C(\bar{\omega}) \cap H_{0}^{1}(\omega)$ and we have

$$
\|u\|_{L^{\infty}} \leq C(\omega)|f|_{H^{1 / 2}}|g|_{H^{1 / 2}}
$$

and

$$
\|u\|_{H^{1}} \leq C(\omega)|f|_{H^{1 / 2}}|g|_{H^{1 / 2}} .
$$

Proof. It suffices to establish the above estimates when $f$ and $g$ are smooth. In this case, $u$ is smooth and we have ${ }^{11}$

$$
\left\{\begin{array}{ll}
-\Delta u=2 \nabla u(f) \cdot \nabla u(g) & \text { in } \omega \\
u=0 & \text { on } \partial \omega
\end{array} .\right.
$$

If we multiply (2.34) by $u$ and use (2.30), we find that

$$
\int_{\omega}|\nabla u|^{2} \leq C(\omega)\|\nabla u\|_{L^{2}}\|\nabla u(f)\|_{L^{2}}\|\nabla u(g)\|_{L^{2}} \leq C(\omega)\|\nabla u\|_{L^{2}}|f|_{H^{1 / 2}}|g|_{H^{1 / 2}},
$$

whence (2.33).

On the other hand, by the proof of Lemma 2.20 , we may write $\nabla u(g)=-\nabla v-c_{j} X_{j}^{\perp}$, with $\|\nabla v\|_{L^{2}} \leq$ $C|g|_{H^{1 / 2}}$ and $\left|c_{j}\right| \leq C|g|_{H^{1 / 2}}$. We find that

$$
\Delta u=2 \operatorname{Jac}(u(f), v)-2 \sum c_{j}(\nabla u(f)) \wedge X_{j} .
$$

Then using Lemma 2.19, we obtain that

$$
\|u\|_{L^{\infty}} \leq C(\omega)\left(\|\nabla u(f)\|_{L^{2}}\|\nabla v\|_{L^{2}}+\sum\left|c_{j}\right|\|\nabla u(f)\|_{L^{2}}\left\|X_{j}\right\|_{L^{\infty}}\right) \leq C(\omega)|f|_{H^{1 / 2}}|g|_{H^{1 / 2}}
$$

From the above, if $f_{n} \rightarrow f$ and $g_{n} \rightarrow g$ in $H^{1 / 2}$, then for the corresponding $u$ 's we have $u_{n} \rightarrow u$ in $H^{1}(\omega)$. This conclusion can be strengthened as follows.

2.22 Lemma. Let $f, g_{n}, g \in H^{1 / 2}(\partial \omega)$ be such that $g_{n} \rightarrow g$ in $H^{1 / 2}$. Then $u\left(f g_{n}\right)-u(f) u\left(g_{n}\right) \rightarrow u(f g)-$ $u(f) u(g)$ strongly in $H^{1}(\omega)$.

As a consequence, if $f_{n} \rightarrow f$ and $g_{n} \rightarrow g$ in $H^{1 / 2}$ then $u\left(f_{n} g_{n}\right)-u\left(f_{n}\right) u\left(g_{n}\right) \rightarrow u(f g)-u(f) u(g)$ strongly in $H^{1}(\omega)$.

Proof. Let $u_{n}:=u\left(f g_{n}\right)-u(f) u\left(g_{n}\right)-u(f g)+u(f) u(g)$. By Lemma 2.21, we have $\left|u_{n}\right| \leq C$ and $u_{n} \rightarrow 0$ in $H^{1}(\omega)$. Thus $u_{n} \nabla u(f) \rightarrow 0$ in $L^{2}(\omega)$. Since $-\Delta u_{n}=2 \nabla u(f) \cdot\left(\nabla u\left(g_{n}\right)-\nabla u(g)\right)$, we find that

$$
\int_{\omega}\left|\nabla u_{n}\right|^{2}=2 \int_{\omega}\left(u_{n} \nabla u(f)\right) \cdot\left(\nabla u\left(g_{n}\right)-\nabla u(g)\right) \rightarrow 0 \quad \text { as } n \rightarrow \infty .
$$

2.23 Remark. Lemma 2.22 can be restated as follows: if $g_{n} \rightarrow g$ weakly in $H^{1 / 2}$, then $\nabla u(f) \cdot \nabla u\left(g_{n}\right) \rightarrow$ $\nabla u(f) \cdot \nabla u(g)$ strongly in $H^{-1}(\omega)$. The above proof leads to the following more general fact: if $u \in H^{1}(\omega)$ and $v_{n} \rightarrow v$ in $H^{1}(\omega)$, with $v_{n}$ harmonic, then $\nabla u \cdot \nabla v_{n} \rightarrow \nabla u \cdot \nabla v$ strongly in $H^{-1}(\omega) .{ }^{12}$

\footnotetext{
${ }^{11}$ For further use, let us note that a byproduct of the proof is that (2.34) still holds for arbitrary $f$ and $g$.

${ }^{12}$ For a cousin assertion when $\omega$ is simply connected, see [24, Lemma 4.2].
} 
2.24 Lemma. Let $f, g_{n} \in H^{1 / 2}\left(\partial \omega ; \mathbb{S}^{1}\right)$. Assume that $g_{n} \rightarrow 1$ in $H^{1 / 2}$. Then

$$
\int_{\omega}\left|\nabla u\left(f g_{n}\right)\right|^{2}=\int_{\omega}|\nabla u(f)|^{2}+\int_{\omega}\left|\nabla u\left(g_{n}\right)\right|^{2}+o(1) \quad \text { as } n \rightarrow \infty .
$$

Equivalently, $\left|f g_{n}\right|_{H^{1 / 2}}^{2}=|f|_{H^{1 / 2}}^{2}+\left|g_{n}\right|_{H^{1 / 2}}^{2}+o(1)$.

Proof. Let $u:=u(f)$ and $v_{n}:=u\left(g_{n}\right)$. In view of Lemma 2.22, (2.36) is equivalent to

$$
\int_{\omega}\left|u \nabla v_{n}+v_{n} \nabla u\right|^{2}=\int_{\omega}|\nabla u|^{2}+\int_{\omega}\left|\nabla v_{n}\right|^{2}+o(1) .
$$

Using the maximum principle Lemma 2.17 combined with the fact that $v_{n} \rightarrow 1$ a.e. in $\omega$ and $\nabla v_{n} \rightarrow 0$ in $L^{2}(\omega)$, we find that

$$
\int_{\omega}\left(u \nabla v_{n}\right) \cdot\left(v_{n} \nabla u\right)=\int_{\omega} \nabla v_{n} \cdot\left(v_{n} \bar{u} \nabla u\right) \rightarrow 0 .
$$

Then (2.37) amounts to

$$
\int_{\omega}|u|^{2}\left|\nabla v_{n}\right|^{2}+\int_{\omega}\left|v_{n}\right|^{2}|\nabla u|^{2}=\int_{\omega}\left|\nabla v_{n}\right|^{2}+\int_{\omega}|\nabla u|^{2}+o(1) .
$$

In turn, (2.38) is easily obtained by combining the following ingredients:

1. $|u(z)| \rightarrow 1$ uniformly as $\operatorname{dist}(z, \partial \omega) \rightarrow 0$ (cf Lemma 2.12).

2. $\int_{K}\left|\nabla v_{n}\right|^{2} \rightarrow 0$ on each compact subset $K \subset \omega$.

3. $\left|v_{n}\right| \leq 1$ (cf Lemma 2.17).

4. $v_{n} \rightarrow 1$ uniformly on compact subsets of $\omega$.

2.25 Remark. The proof of (2.36) is much simpler when $\omega$ is simply connected. Indeed, by conformal invariance of the quantities we consider, we may assume that $\omega=\mathbb{D}$. In this case, if $f=\sum a_{n} e^{\ln \theta}$, then Parseval's identity combined with (2.3) yields

$$
\int_{\mathbb{S}^{1}} \int_{\mathbb{S}^{1}} \frac{|f(x)-f(y)|^{2}}{|x-y|^{2}} d x d y=4 \pi^{2} \sum|n|\left|a_{n}\right|^{2}=4 \pi|f|_{H^{1 / 2}}^{2} .
$$

Thus (2.36) amounts to

$$
\begin{aligned}
\int_{\mathbb{S}^{1}} \int_{\mathbb{S}^{1}} \frac{\left|f(x) g_{n}(x)-f(y) g_{n}(y)\right|^{2}}{|x-y|^{2}} d x d y= & \int_{\mathbb{S}^{1}} \int_{\mathbb{S}^{1}} \frac{|f(x)-f(y)|^{2}}{|x-y|^{2}} d x d y \\
& +\int_{\mathbb{S}^{1}} \int_{\mathbb{S}^{1}} \frac{\left|g_{n}(x)-g_{n}(y)\right|^{2}}{|x-y|^{2}} d x d y+o(1) .
\end{aligned}
$$

Using the fact that $|f|=\left|g_{n}\right|=1$, we obtain

$$
\begin{aligned}
\frac{\left|f(x) g_{n}(x)-f(y) g_{n}(y)\right|^{2}}{|x-y|^{2}} & =\frac{\left|f(x)\left(g_{n}(x)-g_{n}(y)\right)+(f(x)-f(y)) g_{n}(y)\right|^{2}}{|x-y|^{2}} \\
& =\frac{\left|g_{n}(x)-g_{n}(y)\right|^{2}}{|x-y|^{2}}+\frac{|f(x)-f(y)|^{2}}{|x-y|^{2}}+2 F_{n} \cdot G_{n}
\end{aligned}
$$


where $F_{n}(x, y)=\bar{f}(x) g_{n}(y) \frac{f(x)-f(y)}{|x-y|}$ and $G_{n}(x, y)=\frac{g_{n}(x)-g_{n}(y)}{|x-y|}$. Thus (2.40) is equivalent to

$$
\int_{\mathbb{S}^{1}} \int_{\mathbb{S}^{1}} F_{n} \cdot G_{n} \rightarrow 0
$$

We obtain (2.41) using the fact that $F_{n} \rightarrow F(x, y)=\bar{f}(x) \frac{f(x)-f(y)}{|x-y|}$ strongly in $L^{2}\left(\mathbb{S}^{1} \times \mathbb{S}^{1}\right)$ and $G_{n} \rightarrow$ 0 weakly in $L^{2}\left(\mathbb{S}^{1} \times \mathbb{S}^{1}\right) .{ }^{13}$

\section{The basic example}

We discuss here the minimization of $F_{\beta}$ in $\mathscr{G}_{d}$ with $d \in \mathbb{N}^{*} .14$

Recall that a Blaschke product is a map of the form

$$
B_{\alpha, a_{1}, \ldots, a_{d}}(z)=\alpha \prod_{j=1}^{d} \frac{z-a_{j}}{1-\overline{a_{j}} z}, \quad z \in \overline{\mathbb{D}}, d \in \mathbb{N}^{*}, \alpha \in \mathbb{S}^{1}, a_{j} \in \mathbb{D}, \forall j \in \llbracket 1, d \rrbracket .
$$

More specifically, we will call such a product a $d$-Blaschke product. In the special case $d=1$, a Blaschke products reduces to a Moebius transform

$$
M_{\alpha, a}(z)=\alpha \frac{z-a}{1-\bar{a} z}, \quad z \in \bar{D}, \alpha \in \mathbb{S}^{1}, a \in \mathbb{D} .
$$

For further use, let us also define $M_{a}=M_{1, a}$ and the restriction of $M_{a}$ to $\mathbb{S}^{1}$ :

$$
N_{a}: \mathbb{S}^{1} \rightarrow \mathbb{S}^{1}, N_{a}(z)=M_{a}(z), \quad z \in \mathbb{S}^{1} .
$$

3.1 Lemma. Assume that $\beta=0$. Then u minimizes $F_{0}$ in $\mathscr{G}_{d}$ if and only if $u$ is a $d$-Blaschke product.

Proof. We argue as in [5, Section 4.1]. Since $|\nabla u|^{2} \geq 2$ Jac $u$, we have

$$
F_{0}(u)=\frac{1}{2} \int_{\mathbb{D}}|\nabla u|^{2} \geq \int_{\mathbb{D}} \operatorname{Jac} u=\pi \operatorname{deg}\left(u, \mathbb{S}^{1}\right)=\pi d,
$$

the second equality following from (2.8). Equality in (3.2) requires $|\nabla u|^{2}=2$ Jac $u$ a.e., which implies that $u$ is holomorphic. In particular, if $g=\operatorname{tr} u$, then $u=u(g)$.

On the other hand, if $g \in H^{1 / 2}\left(\mathbb{S}^{1} ; \mathbb{S}^{1}\right)$ and if $u=u(g)$ is the harmonic extension of $g$, then Lemma 2.12 implies

$$
\lim _{|z| \rightarrow 1}|u(z)|=1 \text {. }
$$

In order to complete the proof of Lemma 3.1, it suffices to combine (3.3) with the holomorphy of $u$ and with the following well-known result: let $u \in \operatorname{Hol}(\mathbb{D}) .{ }^{15}$ Then

$$
\lim _{|z| \rightarrow 1}|u(z)|=1 \text { uniformly } \Longleftrightarrow u \text { is a Blaschke product. }
$$

\footnotetext{
${ }^{13}$ The first convergence is obtained by combining the fact that $f_{n} \rightarrow f$ in $H^{1 / 2}$ with dominated convergence. The second convergence follows from the fact that $g_{n}-1 \rightarrow 0$ in $H^{1 / 2}$.

${ }^{14}$ The reader may wonder what happens when $d \leq 0$. When $d=0$, it is clear that minimizers of $F_{\alpha}$ are precisely the constants of modulus 1 . The case $d<0$ is obtained from the case $d>0$ by complex conjugation.

${ }^{15}$ We denote by $\operatorname{Hol}(\Omega)$ the class of holomorphic functions in $\Omega$.
} 
For the sake of completeness, we recall the proof of $" \Longrightarrow "$ (implication " $\Longleftarrow "$ being obvious). Let $z_{1}, \ldots, z_{d}$ be the zeroes of $u$ in $\mathbb{D}$, counted with their multiplicities. Let $v(z)=\prod_{j=1}^{d} \frac{z-z_{j}}{1-\overline{z_{j}} z}$ and set $w=\frac{u}{v} \in \operatorname{Hol}(\mathbb{D})$. Then $w \neq 0$ in $\mathbb{D}$ and $\lim _{|z| \rightarrow 1}|w(z)|=1$ uniformly. Thus $w=e^{f}$, where $f \in \operatorname{Hol}(\mathbb{D})$ satisfies $\lim _{|z| \rightarrow 1} \operatorname{Re} f(z)=0$. By the maximum principle, we have $\operatorname{Re} f \equiv 0$, and thus $\operatorname{Im} f \equiv$ const. Therefore, $u=\alpha v$ for some $\alpha \in \mathbb{S}^{1}$.

3.2 Corollary. Let $g \in H^{1 / 2}\left(\mathbb{S}^{1} ; \mathbb{S}^{1}\right)$ have degree $d>0$. Then $|g|_{H^{1 / 2}}^{2} \geq \pi d$, with equality if and only if $g$ is a d-Blaschke product.

Fix now a smooth simply connected domain $\Omega$ and a conformal representation $\Phi: \Omega \rightarrow \mathbb{D}$. Then we define a (generalized) $d$-Blaschke product ${ }^{16}$ by the formula

$$
B_{\alpha, a_{1}, \ldots, a_{d}, \Phi}(z)=\alpha \prod_{j=1}^{d} \frac{\Phi(z)-a_{j}}{1-\overline{a_{j}} \Phi(z)}, \quad z \in \bar{\Omega}, d \in \mathbb{N}^{*}, \alpha \in \mathbb{S}^{1}, a_{j} \in \mathbb{D}, \forall j \in \llbracket 1, d \rrbracket,
$$

and a (generalized) Moebius transform via

$$
M_{\alpha, a, \Phi}(z)=\alpha \frac{\Phi(z)-a}{1-\bar{a} \Phi(z)}, \quad z \in \bar{\Omega}, \alpha \in \mathbb{S}^{1}, a \in \mathbb{D} .
$$

Using Lemma 3.1 and the invariance of the Dirichlet integral under conformal representations, we obtain

3.3 Corollary. The minimizers of $E_{\infty}$ in $\mathscr{E}_{d}$ are precisely the d-Blaschke products.

The next results implies, in particular, that the infimum of $E_{\varepsilon}$ in $\mathscr{E}_{d}$ is not attained when $\varepsilon<\infty$.

3.4 Lemma. Let $\beta \not \equiv 0$. If $d \neq 0$ then $F_{\beta}$ does not attain its minimum in $\mathscr{G}_{d}$.

Proof. Let $u \in \mathscr{G}_{d}$. Then $F_{\beta}(u) \geq F_{0}(u)=\pi d$. We find that $\inf _{\mathscr{G}_{d}} F_{\beta} \geq \pi d$. On the other hand, consider the $d$-Blaschke product $u=\prod_{j=1}^{d} \frac{\Phi-a_{j}}{1-\overline{a_{j}} \Phi}$ and let $a_{j} \rightarrow-1, \forall j$. Then $u \rightarrow 1$ in $H^{1}(\Omega)$, and in particular $F_{\beta}(u) \rightarrow \pi d$. We find that $\inf _{\mathscr{G}_{d}} F_{\beta}=\pi d$. In order to prove that the minimum is not attained, argue by contradiction: assume that $u$ minimizes $F_{\beta}$ in $\mathscr{G}_{d}$. Then $F_{\beta}(u)=F_{0}(u)=\pi d$. Thus $u$ is a $d$-Blaschke product and $\int_{\Omega} \beta\left(1-|u|^{2}\right)^{2}=0$. This is impossible, since $|u|<1$ in $\mathbb{D}$ and thus $\beta\left(1-|u|^{2}\right)^{2} \not \equiv 0$.

We complete this section with the following strong improvement of Lemma 3.1.

3.5 Lemma. The critical points of $F_{0}$ in $\mathscr{G}_{d}$ are precisely:

a) the $d$-Blaschke products if $d>0$.

b) the conjugates of $(-d)$-Blaschke products if $d<0$.

c) constants of modulus 1 if $d=0$.

After our work was completed, we learned that the same result has also been obtained independently by V. Millot and Y. Sire [35].

\footnotetext{
${ }^{16}$ In $\Omega$ and with respect to $\Phi$, but this will be tacitly understood in what follows.
} 
Proof. We rely on the properties of the Hopf differential in arbitrary domains $\Omega \subset \mathbb{R}^{2}$, for which we send the reader to [30, Chapter 4]; in particular, the reader will find there calculations similar to the ones leading to (3.5) or (3.6). If $u: \Omega \rightarrow \mathbb{C}$ is a smooth function, then the Hopf differential $\xi$ of $u$ is defined by the formula

$$
\xi: \Omega \rightarrow \mathbb{C}, \quad \xi(z)=4\left(\partial_{z} u\right)\left(\partial_{z} \bar{u}\right)=\left(\partial_{x} u-\imath \partial_{y} u\right)\left(\partial_{x} \bar{u}-\imath \partial_{y} \bar{u}\right)
$$

If, in addition, $u$ is harmonic, then $\xi$ is holomorphic, and the equality $\xi=0$ is equivalent to either $u$ being holomorphic or $u$ being anti-holomorphic.

We next derive some identities valid when $\Omega$ or $u$ have additional properties. Assume first that $\Omega$ is smooth and that $u$ is smooth near some point $z \in \partial \Omega$, and let $v=v_{x}+\imath v_{y}$ denote the outward normal to $\partial \Omega$. Let $\frac{\partial}{\partial \tau}$ and $\frac{\partial}{\partial v}$ denote respectively the normal and the tangential derivative on $\partial \Omega$ (with respect to the counterclockwise orientation). Using the equalities

$$
\frac{\partial}{\partial x}=v_{x} \frac{\partial}{\partial v}-v_{y} \frac{\partial}{\partial \tau} \text { and } \frac{\partial}{\partial y}=v_{y} \frac{\partial}{\partial v}+v_{x} \frac{\partial}{\partial \tau} u
$$

definition (3.4) can be rewritten as

$$
\xi(z)=\left(v_{x}-\imath v_{y}\right)^{2}\left(\frac{\partial u}{\partial v}-\imath \frac{\partial u}{\partial \tau}\right)\left(\frac{\partial \bar{u}}{\partial v}-\imath \frac{\partial \bar{u}}{\partial \tau}\right)
$$

If, in addition, $u$ is a critical point of $E_{\varepsilon}$ in $\Omega$ (or of $F_{\beta}$ in $\mathbb{D}$ ), then we have $\partial \rho / \partial \tau=0$ on $\partial \Omega$. Therefore, (2.24) combined with (3.5) leads to

$$
\xi=\left(v_{x}-\imath v_{y}\right)^{2}\left[\left(\frac{\partial \rho}{\partial v}\right)^{2}-\left(\frac{\partial \varphi}{\partial \tau}\right)^{2}\right] \quad \text { on } \partial \Omega .
$$

We now complete the proof of Lemma 3.5. Since problem (2.23) with $\beta=0$ is invariant by conformal representations, we may assume that $\Omega=\mathbb{D}$. When $u$ is harmonic, the map $\eta: \mathbb{D} \rightarrow \mathbb{C}, \eta(z)=z^{2} \xi(z)$, is holomorphic. If in addition $\Omega=\mathbb{D}$ and $u$ solves (2.23), then (3.6) becomes

$$
\eta=\left(\frac{\partial \rho}{\partial v}\right)^{2}-\left(\frac{\partial \varphi}{\partial \tau}\right)^{2} \in \mathbb{R} \quad \text { on } \mathbb{S}^{1}
$$

By (3.7), $\eta$ is constant in $\mathbb{D}$. On the other hand, $\eta$ vanishes at the origin. It follows that $\xi \equiv 0$, and therefore $u$ is either holomorphic or anti-holomorphic. Since $|u|=1$ on $\mathbb{S}^{1}$, we obtain the desired conclusion as in the proof of Lemma 3.1.

\section{Moebius and almost Moebius transforms}

In this section, we describe harmonic maps $u$ which are "close" to Moebius maps. Recall that we defined the class

$$
\mathscr{H}_{d}=\left\{g \in H^{1 / 2}\left(\mathbb{S}^{1} ; \mathbb{S}^{1}\right) ; \operatorname{deg} g=d\right\} .
$$

Recall that we denoted by $N_{a}$ the restriction to $\mathbb{S}^{1}$ of the Moebius transform $M_{1, a}$. Recall also Corollary 3.2: for $g \in \mathscr{H}_{1}$ we have $|g|_{H^{1 / 2}}^{2} \geq \pi$, with equality if and only if $g=\alpha N_{a}$ for some $a \in \mathbb{D}$ and $\alpha \in \mathbb{S}^{1}$. 
4.1 Theorem. There exists some $\delta_{0}>0$ and a function $f:\left(0, \delta_{0}\right) \rightarrow(0, \infty)$ such that $\lim _{\delta \rightarrow 0} f(\delta)=0$ with the following property: if $g \in \mathscr{H}_{1}$ satisfies $|g|_{H^{1 / 2}}^{2}<\pi+\delta$ for some $\delta<\delta_{0}$, then:

1. The harmonic extension $u=u(g)$ of $g$ has exactly one zero, $a=a(u)=a(g)$.

2. If we write $g=N_{a} e^{\imath \psi}$ with $\psi \in H^{1 / 2}\left(\mathbb{S}^{1} ; \mathbb{R}\right)$, then $|\psi|_{H^{1 / 2}} \leq f(\delta)$.

3. The map $g \mapsto a$ is continuous.

4. In addition, given $r \in(0,1)$ and $\mu>0$, we may pick $\delta_{0}$ such that $\left\|\alpha u \circ M_{-a}-\mathrm{Id}\right\|_{C^{2}\left(\mathbb{D}_{r}\right)}<\mu$ for some appropriate $\alpha \in \mathbb{S}^{1}$.

Before proceeding to the proof, let us make two comments. First, using repeatedly Lemma 2.6, we find that $\frac{g}{N_{a}}$ has degree zero, and thus we may write $g=N_{a} e^{\imath \psi}$ for some $\psi \in H^{1 / 2}\left(\mathbb{S}^{1} ; \mathbb{R}\right)$. The point in item 2. is that $|\psi|_{H^{1 / 2}}$ is small when $|g|_{H^{1 / 2}}^{2}$ is close to $\pi$. Second, an equivalent and possibly more illuminating formulation of item 4 . is the following: for sufficiently small $\delta_{0}$ and for $g$ as above, we have $\left\|u \circ M_{\alpha, a}^{-1}-\operatorname{Id}\right\|_{C^{2}\left(\mathbb{D}_{r}\right)}<\mu$ for some $\alpha \in \mathbb{S}^{1}$; that is, $u$ is close, in an appropriate sense, to a Moebius transform.

Proof. Step 1. $u$ has at least one zero in $\mathbb{D}$.

Indeed, otherwise we have $u \in C(\mathbb{D} ; \mathbb{C} \backslash\{0\})$ and $\lim _{|z| \rightarrow 1}|u(z)|=1$. Thus $|u| \geq \alpha>0$ for some $\alpha$. By Lemma 2.7 , we may write $u=|u| e^{\imath \varphi}$, with $\varphi \in H^{1}(\Omega ; \mathbb{R})$. The fact that $\operatorname{tr} e^{\imath \varphi}=g$ combined with the degree formula (2.8) leads to the contradiction

$$
1=\operatorname{deg}\left(g, \mathbb{S}^{1}\right)=\frac{1}{\pi} \int_{\mathbb{D}} \operatorname{Jac}\left(e^{\imath \varphi}\right)=0 .
$$

Let now $a \in \mathbb{D}$ be one of the (possibly several) zeroes of $u$. Set $v=u \circ M_{-a}=u \circ M_{a}^{-1}$. Then $v$ is harmonic, $v(0)=0$, and $v_{\mathbb{S}^{1}}=h$, with $h=g \circ N_{-a}$. In addition, we have $|h|_{H^{1 / 2}}=|g|_{H^{1 / 2}}$, by conformal invariance of the $H^{1 / 2}$-semi-norm.

Step 2. Proof of 4 .

Argue by contradiction: there are some $\mu>0, r \in(0,1)$ such that

$$
\inf _{\alpha \in \mathbb{S}^{1}}\left\|\alpha v_{n}-\operatorname{Id}\right\|_{C^{2}\left(\mathbb{D}_{r}\right)} \geq \mu
$$

for some sequence $\left(v_{n}\right)$ of harmonic maps such that

$$
\left\{\begin{array}{l}
v_{n}(0)=0 \\
h_{n}:=v_{n \mid \mathbb{S}^{1}} \text { has modulus } 1 \text { and degree } 1 . \\
\left|h_{n}\right|_{H^{1 / 2}}^{2} \leq \pi+\frac{1}{n}
\end{array}\right.
$$

We find that, possibly up to a subsequence, $v_{n} \rightarrow v$ in $C_{l o c}^{\infty}(\mathbb{D})$, and $h_{n} \rightarrow h \in H^{1 / 2}\left(\mathbb{S}^{1} ; \mathbb{S}^{1}\right)$. In addition, we have $v=u(h)$. The limit $v$ satisfies

$$
\left\{\begin{array}{l}
\|\alpha v-\operatorname{Id}\|_{C^{2}\left(\mathbb{D}_{r}\right)} \geq \mu, \forall \alpha \in \mathbb{S}^{1} \\
v(0)=0 \\
|h|_{H^{1 / 2}}^{2} \leq \pi
\end{array} .\right.
$$


We consider first the case where $\operatorname{deg}\left(h, \mathbb{S}^{1}\right)=1$. Since $|h|_{H^{1 / 2}}^{2} \leq \pi$, Corollary 3.2 combined with $v(0)=0$ leads to $v=\gamma$ Id for some $\gamma \in \mathbb{S}^{1}$ and this is impossible.

Thus $\operatorname{deg}\left(h, \mathbb{S}^{1}\right) \neq 1$. Then the Price Lemma 2.18 gives:

$$
\pi=\lim \frac{1}{2} \int_{\mathbb{D}}\left|\nabla v_{n}\right|^{2} \geq \frac{1}{2} \int_{\mathbb{D}}|\nabla v|^{2}+\pi\left|\operatorname{deg}\left(h_{n}, \mathbb{S}^{1}\right)-\operatorname{deg}\left(h, \mathbb{S}^{1}\right)\right|=\frac{1}{2} \int_{\mathbb{D}}|\nabla v|^{2}+\pi\left|1-\operatorname{deg}\left(h, \mathbb{S}^{1}\right)\right| .
$$

Therefore, $v$ is a constant of modulus 1 . This contradicts $v(0)=0$.

In the remaining part of the proof, we assume that $|g|_{H^{1 / 2}}^{2} \leq \pi+\delta$, where $\delta<\delta_{0}$ and $\delta_{0}$ is to be chosen later.

Step 3. For $0<s<r<1$ and for sufficiently small $\delta_{0}$ (depending on $s$ and $r$ ), we have $|v| \geq s$ on $\mathbb{D} \backslash \mathbb{D}_{r}$. Indeed, let $\mu>0$ and let $R \in(r, 1)$ to be specified later. By Step 2 . we have

$$
\frac{1}{2} \int_{\mathbb{D}_{R}}|\nabla v|^{2} \geq \pi R^{2}-\mu \text { and }|v(x)| \geq|x|-\mu \text { in } \overline{\mathbb{D}}_{R}
$$

provided $\delta_{0}$ is sufficiently small. In particular, we have

$$
\frac{1}{2} \int_{\mathbb{D} \backslash \overline{\mathbb{D}}_{R}}|\nabla v|^{2} \leq \pi-\pi R^{2}+\mu+\delta_{0} \text { and }|v| \geq R-\mu \text { on } \partial\left(\mathbb{D} \backslash \overline{\mathbb{D}}_{R}\right) .
$$

We next invoke the following special cases of [24, Theorem 3.6] combined with [24, Example 3.5 c)].

4.2 Lemma. 1. Let $0<R<1$ and set $\omega:=\mathbb{D} \backslash \overline{\mathbb{D}}_{R}$. Let $v \in H^{1}(\mathbb{D})$ be a complex-valued harmonic function. Assume that $t \leq|v| \leq 1$ on $\partial \omega$, for some $t \geq 0$, and let $s=\underset{\omega}{\inf }|v|$. Then we have

$$
\int_{\omega}|\nabla v|^{2} \geq 4 \mathscr{A}_{s, t}
$$

where $\mathscr{A}_{s, t}$ is the area of the region $\{z \in \mathbb{D} ;|z| \leq t, \operatorname{Re} z \geq s\}$.

2. Same conclusion if $\omega=\mathbb{D}$.

3. Same conclusion if $\omega=\mathbb{D}$ or $\mathbb{D} \backslash \overline{\mathbb{D}}_{R}$ and if $v$ minimizes $F_{\beta}$ in $\mathbb{D}$ with respect with its own boundary condition.

4. Special case: if $v$ is as above and satisfies $|v|=1$ on $\mathbb{S}^{1}$, and if $\int_{\mathbb{D}}|\nabla v|^{2} \leq c<2 \pi$, then there exists some $\lambda=\lambda(c)>0$ such that $|v| \geq \lambda$ in $\mathbb{D}$.

Step 3 continued. Note that $\mathscr{A}_{s, t}$ does not depend on $R$. Therefore, by combining item 1 . of Lemma 4.2 with (4.3), we obtain the following: if $R$ is sufficiently close to 1 and if $\mu$ and $\delta_{0}$ are sufficiently small, then $|v| \geq s$ in $\mathbb{D} \backslash \overline{\mathbb{D}}_{R}$. We conclude by combining this fact with the second assertion in (4.2).

Step 4. The map $u$ has exactly one zero.

Indeed, by combining Step 3. with Step 2., we find that $v$ has exactly one zero for small $\delta_{0}$ (and that this zero is located near the origin). Whence the conclusion for $u$.

Step 5. The continuity of $g \mapsto a$.

This follows essentially from uniqueness, but some care is needed, since in principle zeroes could escape to the boundary. Let $g_{n} \rightarrow g$ in $H^{1 / 2}\left(\mathbb{S}^{1} ; \mathbb{S}^{1}\right)$. Then there is some $r>0$ independent of $n$ such

$$
\left|u\left(g_{n}\right)\right| \geq \frac{1}{2} \quad \text { in } \mathbb{D} \backslash \overline{\mathbb{D}}_{r}, \forall n .
$$


This uniform version of (2.15) can be easily obtained by following the proof of [21, Theorem A3.2]. The continuity of $g \mapsto a$ is a straightforward consequence of (4.4).

Step 6. We prove 2.

Since ||$_{H^{1 / 2}}$ is invariant by composition with restrictions to $\mathbb{S}^{1}$ of Moebius transforms and by multiplication with unimodular constants, we may assume that $u(0)=0$, and that the conclusion of item 4. holds with $a=0$ and $\alpha=1$. Let $\theta$ denote the polar angle and let $r \in(1 / 2,1)$. By item 4 ., for small $\delta$ we are in position to apply Corollary 2.9 with $\omega=\mathbb{D} \backslash \overline{\mathbb{D}}_{r}, u_{1}=\mathrm{Id}, u_{2}=u=u(g)$. Therefore, for small $\delta$ we may write, in $\mathbb{D} \backslash \overline{\mathbb{D}}_{r}, u=\operatorname{Id} \eta e^{\imath \varphi}=|u| e^{\imath(\theta+\varphi)}$, with $\eta, \varphi \in H^{1}\left(\mathbb{D} \backslash \overline{\mathbb{D}}_{r} ; \mathbb{R}\right)$. By Step 3. and the maximum principle Lemma 2.17, we can assume that $1 / 2 \leq|u| \leq 1$.

We denote by $o(1)$ a quantity which tends to 0 uniformly in $g$ as $\delta \rightarrow 0$. We will next prove the estimate

$$
\|\nabla \varphi\|_{L^{2}\left(\mathbb{D} \backslash \overline{\mathbb{D}}_{r}\right)}=o(1) .
$$

Assuming (4.5) proved for the moment, item 2. follows from (4.5) by taking the $H^{1 / 2}$ semi-norm of the trace $\psi$ of $\varphi$ on $\mathbb{S}^{1}$.

In order to prove (4.5), we first note that $\|u-\operatorname{Id}\|_{H^{1}}=o(1)$. Indeed, consider a sequence $\left(u_{n}\right)$, $u_{n}=u\left(g_{n}\right)$, such that $\delta_{n}:=\left|g_{n}\right|_{H^{1 / 2}}^{2}-\pi \rightarrow 0$ as $n \rightarrow \infty$ and that the conclusion of item 4 . holds with $a=0$ and $\alpha=1$. Then we have $u_{n} \rightarrow$ Id weakly in $H^{1}(\mathbb{D})$, and

$$
\frac{1}{2} \int_{\mathbb{D}}\left|\nabla u_{n}\right|^{2}=\left|g_{n}\right|_{H^{1 / 2}} \rightarrow \pi=\frac{1}{2} \int_{\mathbb{D}}|\nabla \mathrm{Id}|^{2} \quad \text { as } n \rightarrow \infty .
$$

Therefore $\left\|u_{n}-\mathrm{Id}\right\|_{H^{1}} \rightarrow 0$. Next we write

$$
|\nabla \varphi|=\left|\nabla \frac{|\mathrm{Id}| u}{|u| \mathrm{Id}}\right| \quad \text { in } \mathbb{D} \backslash \overline{\mathbb{D}}_{r},
$$

and obtain (4.5) by combining the fact that $\|u-\operatorname{Id}\|_{H^{1}}=o(1)$ with the pointwise bounds $1 / 2 \leq|u| \leq 1$ and $1 / 2 \leq|\mathrm{Id}| \leq 1$ on $\mathbb{D} \backslash \overline{\mathbb{D}}_{r}$.

4.3 Remark. Theorem 4.1 comes with no estimate of the function $f(\delta)$ in item 2 . It would be interesting to have a quantitative form of this result.

We continue with a result similar to Theorem 4.1, in which a loss of information on the zero $a$ of $u(g)$ is compensated by the control of the phase $\psi$ at higher energy levels of $g$.

4.4 Theorem. There exists a non increasing function $h:(0, \pi] \rightarrow(0, \infty)$ such that: if $g \in \mathscr{H}_{1}$ satisfies $|g|_{H^{1 / 2}}^{2} \leq 2 \pi-\delta$ for some $\delta \in(0, \pi]$, then:

1. We may write $g=N_{a} e^{\imath \psi}$ for some $\psi \in H^{1 / 2}\left(\mathbb{S}^{1} ; \mathbb{R}\right)$ such that $|\psi|_{H^{1 / 2}} \leq h(\delta)$.

2. In addition, we may take the point a to be a zero of $u(g)$, and the zeroes of $u(g)$ are mutually close, in the following sense: there exist some $R=R(\delta) \in(0,1)$ and $\mu=\mu(\delta) \in(0,1)$ such that, if a is a zero of $u(g)$, then $|u(g)| \geq \mu$ in $\mathbb{D} \backslash M_{a}^{-1}\left(\overline{\mathbb{D}}_{R}\right)$.

Before proceeding to the proof, let us note the following more informative form of item 2.:

$$
\text { if } u(g)(0)=0 \text {, then }|u(g)| \geq \mu \text { outside } \overline{\mathbb{D}}_{R} \text {. }
$$


Proof. As in the proof of Theorem 4.1, Step 6., it suffices to establish item 2. in the special case where $u(g)(0)=0$. We argue by contradiction: we assume that there exist a sequence $\left(g_{n}\right) \subset \mathscr{H}_{1}$ and a sequence $\left(z_{n}\right) \subset \mathbb{D}$ such that:

$$
\left|g_{n}\right|_{H^{1 / 2}}^{2} \leq 2 \pi-\delta,\left|z_{n}\right| \rightarrow 1, u_{n}:=u\left(g_{n}\right) \text { satisfies } u_{n}(0)=0 \text { and }\left|u_{n}\left(z_{n}\right)\right| \rightarrow 0 .
$$

Possibly after passing to a subsequence, we may also assume that, for some $D \in \mathbb{Z}, g \in \mathscr{H}_{D}$ and $u=u(g)$, we have

$$
u_{n} \rightarrow u \text { in } C_{l o c}^{\infty}(\mathbb{D}), u(0)=0 .
$$

We first prove that $D=1$. Indeed, by the argument leading to (4.1) we have

$$
2 \pi>2 \pi-\delta \geq \frac{1}{2} \int_{\mathbb{D}}|\nabla u|^{2}+\pi|1-D| .
$$

By combining (4.9) with Corollary 3.2, we find that $D=0$ or $D=1$. If $D=0$, then $\int_{\mathbb{D}}|\nabla u|^{2} \leq 2(\pi-\delta)$. This inequality combined with Lemma 4.24. implies that $|u| \geq \lambda$ in $\mathbb{D}$ for some $\lambda>0$. This contradicts (4.8). Thus $D=1$.

Let $s \in(0,1)$ to be chosen later. By Lemma 2.12, there exists some $R \in(0,1)$ such that $|u(z)|>s$ on $C_{R}$. Then, thanks to (4.8), we have $\left|u_{j}\right| \geq s$ on $C_{R}$ when $j$ is large. On the other hand, we have $\operatorname{deg}\left(u, C_{R}\right)=1$ provided $R$ is chosen sufficiently close to 1 ; this follows by combining Lemma 2.2 with Lemma 2.12. By (4.8), for large $n$ we have $\operatorname{deg}\left(u_{n}, C_{R}\right)=1$. Using (2.10), we find that

$$
\frac{1}{2} \int_{\mathbb{D}_{R}}\left|\nabla u_{n}\right|^{2} \geq \pi s^{2} .
$$

On the other hand, since $\left|u_{n}\left(z_{n}\right)\right| \rightarrow 0$ and $\left|u_{n}\right| \geq s$ on $\partial\left(\mathbb{D} \backslash \bar{D}_{R}\right)$, we obtain, by applying Lemma 4.2 1., that

$$
\liminf _{n \rightarrow \infty} \frac{1}{2} \int_{\mathbb{D}_{\mathbb{D}_{R}}}\left|\nabla u_{n}\right|^{2} \geq \pi s^{2} .
$$

For $s$ sufficiently close to 1 , we obtain a contradiction by combining (4.10) with (4.11).

Once the existence of $R$ and $\mu$ as in item 2. is established, item 1. is obtained as follows. By conformal invariance, we may assume that $u(0)=0$. By Lemma 2.2, we have $\operatorname{deg}\left(u, C_{R}\right)=1$. Arguing as at the beginning of Step 6 . in the proof of Theorem 4.1, we may globally write, in $\mathbb{D} \backslash \overline{\mathbb{D}}_{R}$,

$$
u=|u| e^{\imath(\theta+\varphi)} .
$$

By (2.11), (4.12) and the bound $\int_{\mathbb{D}}|\nabla u|^{2} \leq 4 \pi$, we obtain that

$$
\int_{\mathbb{D} \backslash \overline{\mathbb{D}}_{R}}|\nabla \varphi|^{2} \leq C .
$$

Item 1. follows from (4.13).

4.5 Remark. It is not clear whether the restriction $|g|_{H^{1 / 2}}^{2}<2 \pi$ is optimal in Theorem 4.4. However, some bound is required. Here is an example with a sketch of proof. Let $g=g_{b, c, d}=\frac{N_{b} N_{c}}{N_{d}}$. Then $|g|_{H^{1 / 2}}^{2} \leq 9 \pi$. If we let, say, $b \rightarrow 1, c \rightarrow-1$ and $d \rightarrow \imath$, then we claim that there exists no $a=a(b, c, d)$ 
such that Theorem 4.41 . holds. Argue by contradiction: otherwise, after passing to a subsequence, we have $a \rightarrow \tilde{a} \in \overline{\mathbb{D}}$. The limit $\tilde{a}$ cannot be close to $1,-1$ and $\imath$ at the same time; say that we have $\tilde{a} \neq 1$. Then, near $z=1, \frac{g}{N_{a}}$ is close to $\alpha N_{b}$ for some $\alpha \in \mathbb{S}^{1}$. Using the fact that the $H^{1 / 2}$-semi-norm of the phase of $N_{b}$ (computed near $z=1$ ) tends to $\infty$ as $b \rightarrow 1$, and the fact that the phase is unique modulo $2 \pi$ [14], we find that Theorem 4.4 does not hold for $g$ as above.

The next result explains that, at low energy, the lack of weak compactness of the class $\mathscr{H}_{1}$ is due solely to Moebius transforms.

4.6 Corollary. Let $t<2 \pi$ and let $\mathscr{K}_{1}^{(t)}=\left\{g \in \mathscr{H}_{1} ;|g|_{H^{1 / 2}}^{2} \leq t\right\}$.

Then $\mathscr{K}_{1}^{(t)}$ is weakly closed modulo Moebius transforms: If $\left(g_{n}\right) \subset \mathscr{K}_{1}^{(t)}$, then there exists $N_{a_{n}}$ such that the sequence $\left(g_{n} \circ N_{a_{n}}^{-1}\right)$ is weakly compact in $\mathscr{K}_{1}^{(t)}$.

In addition, we may take $a_{n}$ to be any zero of $u\left(g_{n}\right)$.

In particular, for every $t<2 \pi$ and $a_{0} \in \mathbb{D}$, the class

$$
\left\{g \in \mathscr{H}_{1} ;|g|_{H^{1 / 2}}^{2} \leq t, u(g) \text { vanishes at } a_{0}\right\}
$$

is weakly compact.

We continue by presenting another consequence of Theorem 4.4.

\subsection{Lemma. Let}

$$
\beta \in L^{\infty}(\mathbb{D}), \beta \geq 0, \beta \not \equiv 0 .
$$

Consider a Lebesgue point $a_{0} \in \mathbb{D}$ of $\beta$ at which the approximate limit of $\beta$ is $b>0$. Then

$$
c\left(\beta, a_{0}\right):=\inf \left\{F_{\beta}(v) ; g=\operatorname{tr} v \in \mathscr{H}_{1}, u(g) \text { vanishes at } a_{0}\right\}>\pi \text {. }
$$

Proof. If $c(\beta, a) \geq 2 \pi$, there is nothing to prove. Otherwise, we apply Corollary 4.6 and obtain that the minimum is attained in (4.15). Argue by contradiction and assume that $c\left(\beta, a_{0}\right)=\pi$. If $v$ attains the minimum in (4.15) then, by Corollary $3.2, v=M_{\alpha, a}$ and

$$
\int_{\mathbb{D}} \beta\left(1-|v|^{2}\right)^{2}=0 .
$$

Our choice of $a$ implies that

$$
\lim _{r \rightarrow 0} f_{B(a, r)} \beta\left(1-|v|^{2}\right)^{2}=b\left(1-|v|^{2}\right)^{2}(a) .
$$

We obtain a contradiction by combining (4.16) and (4.17).

Though this will not be used in the subsequent analysis, we found useful to mention that part of Theorem 4.1 still holds for higher degrees. ${ }^{17}$

\footnotetext{
${ }^{17}$ Or for negative degrees, but this is simply obtained by complex conjugation.
} 
4.8 Theorem. Let $d \in \mathbb{N}$. Then there exists some $\delta_{0}>0$ and a function $f:\left(0, \delta_{0}\right) \rightarrow(0, \infty)$ such that $\lim _{\delta \rightarrow 0} f(\delta)=0$ with the following property: if $g \in \mathscr{H}_{d}$ satisfies $|g|_{H^{1 / 2}}^{2}<\pi d+\delta$ for some $\delta<\delta_{0}$, then we can write $g=N_{a_{1}} \ldots N_{a_{d}} e^{\imath \psi}$ for some $a_{1}, \ldots, a_{d} \in \mathbb{D}$ and for some $\psi \in H^{1 / 2}\left(\mathbb{S}^{1} ; \mathbb{R}\right)$ such that $|\psi|_{H^{1 / 2}} \leq f(\delta)$.

Consequently, if $u \in \mathscr{G}_{d}$ is a harmonic function and if $\frac{1}{2} \int_{\mathbb{D}}|\nabla u|^{2}<\pi d+\delta$, with $\delta<\delta_{0}$, then $u=$ $B_{1, a_{1}, \ldots, a_{d}} u\left(e^{\imath \psi}\right)+w$, where $\psi \in H^{1 / 2}\left(\mathbb{S}^{1} ; \mathbb{R}\right), w \in H_{0}^{1}(\mathbb{D} ; \mathbb{C}),|\psi|_{H^{1 / 2}} \leq f(\delta)$ and $\|w\|_{H^{1}} \leq h(\delta)$. Here, $h(\delta) \rightarrow 0$ as $\delta \rightarrow 0$.

Proof. The last part of the theorem follows by combining the first part of the theorem with Lemma 2.21 .

In order to prove the first part, it suffices to establish the following fact: if $\left(g_{n}\right) \subset \mathscr{H}_{d}$ is such that $\left|g_{n}\right|_{H^{1 / 2}}^{2} \rightarrow \pi d$, then, possibly up to a subsequence and for large $n$, we may write $g_{n}=N_{a_{1}(n)} \ldots N_{a_{d}(n)} e^{\imath \psi_{n}}$, with $\left|\psi_{n}\right|_{H^{1 / 2}} \rightarrow 0$ as $n \rightarrow \infty$. The proof of this fact is by induction on $d$.

Step 1. The case where $d=0$.

By Lemma 2.13, we have $\left|u\left(g_{n}\right)\right| \rightarrow 1$ uniformly in $\mathbb{D}$. By Lemma 2.7 and (2.11), for large $n$ we may write $u\left(g_{n}\right)=\rho_{n} e^{\imath \varphi_{n}}$, with $\nabla \varphi_{n} \rightarrow 0$ in $L^{2}(\mathbb{D})$. We conclude by letting $\psi_{n}=\operatorname{tr} \varphi_{n}$.

Step 2. The case where $d \geq 1$.

By Step 1. in the proof of Theorem 4.1, the map $u\left(g_{n}\right)$ has to vanish somewhere. Since our hypotheses and conclusions are invariant by conformal transforms, we may assume that $u\left(g_{n}\right)(0)=0$. Up to a subsequence, we have $g_{n} \rightarrow g$, with $g \in \mathscr{H}_{D}$ for some $D \in \mathbb{Z}$. By combining (2.9) with the Price Lemma 2.18, we find that $D \in \llbracket 0, d \rrbracket$ and that $|g|_{H^{1 / 2}}^{2}=\pi D$. By Corollary 3.2, $u(g)$ is a $D$-Blaschke product. Since in addition we have $u(g)(0)=0$, we find that $D \in \llbracket 1, d \rrbracket$. If $D=d$, then we actually have $g_{n} \rightarrow g$ strongly in $H^{1 / 2},{ }^{18}$ and thus $g_{n} / g \rightarrow 1 .{ }^{19}$ In this case, Step 1 . implies that $g_{n}=g e^{\imath \psi_{n}}$, with $\left|\psi_{n}\right|_{H^{1 / 2}} \rightarrow 0$, and we are done.

We next turn to the more delicate case where $D<d$. Let $h_{n}:=g_{n} / g$, so that $h_{n} \in \mathscr{H}_{d-D}$ and $h_{n} \rightarrow 1$. By Lemma 2.24 combined with the fact that $|g|_{H^{1 / 2}}^{2}=\pi D$, we have $\left|h_{n}\right|_{H^{1 / 2}}^{2} \rightarrow \pi(d-D)$. By the induction hypothesis, ${ }^{20}$ we may write, for large $n, h_{n}=N_{a_{1}(n)} \ldots N_{a_{d-D}(n)} e^{i \psi_{n}}$, with $\left|\psi_{n}\right|_{H^{1 / 2}} \rightarrow 0$. We obtain the desired conclusion by noting that $g_{n}=g h_{n}$ and that $g$ is the trace of a $D$-Blaschke product.

\section{Mountain pass approach}

In this section and in Sections 8 and 9 , we will prove existence of (almost) critical points of $F_{\beta}$ in different situations. Each case we examine will require a different functional setting; however the general strategy is the same, and is based on the existence of a mountain pass geometry. So let us start by recalling the standard mountain pass framework; see e.g. [34, Chapter 4]. This framework requires five objects and an inequality. The five objects are: two compact metric spaces $K_{0} \subset K$, a Banach space or Banach manifold $X$, a map $J: X \rightarrow \mathbb{R}$ (usually assumed $C^{1}$ ), and a fixed continuous

\footnotetext{
${ }^{18}$ Since $g_{n} \rightarrow g$ and $|g|_{H^{1 / 2}}^{2}=\lim \left|g_{n}\right|_{H^{1 / 2}}^{2}$.

${ }^{19}$ Here, we use the fact that multiplication is continuous in $H^{1 / 2}\left(\mathbb{S}^{1} ; \mathbb{S}^{1}\right)$. This is folklore and is a special case of the following more general fact. If $u_{n}, u, v \in W^{s, p} \cap L^{\infty}$, with $1 \leq p<\infty$ and $s>0$, and if $u_{n} \rightarrow u$ in $W^{s, p}$ and $\left\|u_{n}\right\|_{L^{\infty}} \leq C$, then $u_{n} v \rightarrow u v$ in $W^{s, p}$.

${ }^{20}$ Recall that $D \geq 1$ and thus $d-D<d$.
} 
map $\chi \in C\left(K_{0} ; X\right) .{ }^{21}$ We associate to these objects the minimization problem

$$
c=\inf \left\{\max _{K} J \circ F ; F \in M\right\}, \quad \text { where } M=\left\{F \in C(K ; X) ; F=\chi \text { on } K_{0}\right\} .
$$

Mountain pass geometry is characterized by the strict inequality

$$
c>c_{1}=\max _{t \in K_{0}} J(\chi(t))
$$

We next recall the Mountain Pass Theorem of Ambrosetti and Rabinowitz [1], which will be used in the following form [34, Theorem 4.3, p. 77].

5.1 Theorem. Let $K_{0}, K, J, X$ and $\chi$ be as above. Assume that (5.2) holds. Assume that $X$ is a Banach space and that $J \in C^{1}(X ; \mathbb{R})$. Then for every $\delta>0$ there exists $x_{\delta} \in X$ such that

$$
c-\delta \leq J\left(x_{\delta}\right) \leq c+\delta
$$

and

$$
\left\|J^{\prime}\left(x_{\delta}\right)\right\| \leq \sqrt{\delta}
$$

Equivalently, there exists a sequence

$$
\left(x_{n}\right) \subset X \text { such that } J\left(x_{n}\right) \rightarrow c \text { and } J^{\prime}\left(x_{n}\right) \rightarrow 0 \text { as } n \rightarrow \infty \text {. }
$$

The same holds when $X$ is a Banach manifold and $J \in C^{1}$.

A sequence as in (5.5) is a "Palais-Smale sequence", and the $x_{n}$ 's are "almost critical points of $J$ ". ${ }^{22}$ Under the additional assumption

$$
(\mathrm{PS})_{\mathrm{c}} \text { each sequence }\left(x_{n}\right) \text { satisfying (5.5) contains a convergent subsequence }
$$

(the $(\mathrm{PS})_{\mathrm{c}}$ ) condition of Brezis, Coron and Nirenberg [18]), (5.2) leads to the existence of a critical point $x$ of $J$ such that $J(x)=c$.

In practice, we will always take

$$
K=\bar{D}_{r}, K_{0}=C_{r} \quad \text { for some appropriate } r \in(0,1) \text {. }
$$

The underlying idea (in this section and in Section 8) is to create mountain pass geometry by letting $\chi(a)=M_{a}, \forall a \in C_{r}$, and $J=F_{\beta}$. While the above choice of $\chi$ is not straightforward, and is one of the main ideas of this paper, the natural choices for $J$ and $X$ are $J=F_{\beta}$ and $X=\mathscr{G}$ (the set of complexvalued $H^{1}$ maps with modulus 1 on the boundary) or possibly $X=\mathscr{G}_{1}$. However, $\mathscr{G}$ and $\mathscr{G}_{1}$ do not have a straightforward manifold structure. In this section, we present a first way of circumventing this difficulty. The approach we develop here allows us to prove the main result of this paper (Theorem 1.1), but has the drawback of working only for large values of $\varepsilon .^{23}$

Thus we address the question of the existence of critical points under the assumption that $\beta$ is small. $^{24}$ More specifically, we assume that the first eigenvalue $\lambda_{1}(-\Delta-\beta)$ of the operator $-\Delta-\beta$ satisfies

$$
\lambda_{1}(-\Delta-\beta)>0
$$

\footnotetext{
${ }^{21}$ In order to distinguish the different constructions we will perform later, we will denote the corresponding function spaces $X$ by $X^{*}$, respectively $X^{\sharp}$. We distinguish similarly the other objects.

${ }^{22}$ At the level $c$, but this will be omitted.

${ }^{23} \mathrm{~A}$ second, more subtle, approach will be presented in the next section.

${ }^{24}$ Of particular interest for us is the fact that the assumption (5.8) is satisfied when in the original problem of finding critical points for $E_{\varepsilon}$ we take a large $\varepsilon$.
} 
i.e., there is some $\delta>0$ such that

$$
(1-\delta) \int_{\mathbb{D}}|\nabla v|^{2} \geq \int_{\mathbb{D}} \beta v^{2}, \quad \forall v \in H_{0}^{1}(\mathbb{D} ; \mathbb{R}) .
$$

For such $\beta$ and fixed boundary condition $g \in H^{1 / 2}\left(\mathbb{S}^{1} ; \mathbb{C}\right)$, the energy functional $F_{\beta}$ is continuous, coercive and strictly convex in the affine space

$$
H_{g}^{1}(\mathbb{D} ; \mathbb{C})=\left\{u \in H^{1}(\mathbb{D} ; \mathbb{C}) ; \operatorname{tr} u=g\right\} .
$$

Therefore, $F_{\beta}$ has exactly one critical point in the above space. Equivalently, the problem

$$
\begin{cases}-\Delta u=\beta u\left(1-|u|^{2}\right) & \text { in } \mathbb{D} \\ u=g & \text { on } \mathbb{S}^{1}\end{cases}
$$

has a unique solution, which we denote $T(g)$.

With this in mind, the role of the space $X$ is played by $X^{*}=H^{1 / 2}\left(\mathbb{S}^{1} ; \mathbb{R}\right)$, and the role of the functional $J$ by

$$
J^{*}: X^{*} \rightarrow \mathbb{R}, J^{*}(\psi)=F_{\beta}\left(T\left(N_{0} e^{\imath \psi}\right)\right), \quad \forall \psi \in X^{*}
$$

Recall that we let $K=\overline{\mathbb{D}}_{r}$ and $K_{0}=C_{r}$, where $0<r<1$. It remains to define the map $\chi^{*}$ playing the role of $\chi$. For this purpose, we note the following. If $a \in C_{r}$, then the restriction $N_{a}$ of $M_{a}$ to $\mathbb{S}^{1}$ can be written as $N_{a}=N_{0} e^{\imath \psi_{a}}=e^{\imath\left(\theta+\psi_{a}\right)}$ for some $\psi_{a} \in H^{1 / 2}\left(\mathbb{S}^{1} ; \mathbb{R}\right)$. The idea is to let, for any $a \in C_{r}$, $\chi^{*}(a)=\psi_{a}$. The next result shows that, when $\psi_{a}$ is properly chosen, $\chi^{*}$ is a continuous map from $C_{r}$ into $X^{*}$.

5.2 Lemma. There exists a map $H \in C^{\infty}\left(\mathbb{D} ; C^{\infty}\left(\mathbb{S}^{1} ; \mathbb{R}\right)\right)$ such that $N_{a}=N_{0} e^{\imath H(a)}, \forall a \in \mathbb{D}$.

Proof. Let $g_{a}=N_{a} / N_{0}$ and let

$$
F(a, \cdot)=g_{a} \wedge \frac{\partial g_{a}}{\partial \tau}=-\imath \overline{g_{a}} \frac{\partial g_{a}}{\partial \tau} \cdot 25
$$

Then clearly $F \in C^{\infty}\left(\mathbb{D} \times \mathbb{S}^{1} ; \mathbb{R}\right)$, and $\int_{\mathbb{S}^{1}} F(a, z) d s_{z}=0$. Therefore, if we let $\eta(a, \cdot)$ be the primitive of $F(a, \cdot)$ with zero average, then $\eta$ is smooth in both variables. In addition, we have

$$
\frac{\partial}{\partial \tau}\left(g_{a} e^{-\imath \eta(a, \cdot)}\right)=g_{a} e^{-\imath \eta(a, \cdot)}\left(\overline{g_{a}} \frac{\partial}{\partial \tau} g_{a}-\imath \frac{\partial}{\partial \tau} \eta(a, \cdot)\right)=0
$$

i.e., $g_{a} e^{-\imath \eta(a, \cdot)}$ is constant. Define $L(a):=g_{a}(1) e^{-\imath \eta(a, 1)}$. Then $L \in C^{\infty}\left(\mathbb{D} ; \mathbb{S}^{1}\right)$, and thus we may write $L=e^{l \zeta}$ for some $\zeta \in C^{\infty}(\mathbb{D} ; \mathbb{R})$. Finally, if we let $H=\eta+\zeta$, then $H$ has all the required properties.

In what follows, we let $\psi_{a}=H(a), a \in C_{r}$.

We next prove that we do have a mountain pass geometry.

5.3 Lemma. Assume (4.14) and (5.8). Then, for $r$ sufficiently close to 1, we have

$$
c^{*}:=\inf \left\{\max _{\overline{\mathbb{D}}_{r}} J^{*} \circ F ; F \in C\left(\overline{\mathbb{D}}_{r} ; H^{1 / 2}\left(\mathbb{S}^{1} ; \mathbb{R}\right)\right), F=\chi^{*} \text { on } C_{r}\right\}>c_{1}^{*}:=\max _{C_{r}} J^{*} \circ \chi^{*}
$$

\footnotetext{
${ }^{25}$ The last identity is easily checked using the fact that $\left|g_{a}\right|^{2}=1$, and thus $g_{a} \cdot \frac{\partial g_{a}}{\partial \tau}=0$.
} 
Proof. It is easy to see that $\left|M_{a}\right| \rightarrow 1$ in $L^{4}(\mathbb{D})$ as $|a| \rightarrow 1$, and thus $c_{1}^{*} \rightarrow \pi$ as $r \rightarrow 1$. We claim that, if $r$ is sufficiently large, then $c^{*}$ is bounded from below by a constant larger than $\pi$; this implies (5.10).

In order to prove the claim, we fix a Lebesgue point $a_{0}$ of $\beta$ where the approximate limit of $\beta$ is (strictly) positive. We assume that $c^{*}<\pi+\delta_{0}$, with $\delta_{0}$ as in Theorem 4.1, for otherwise we are done. Let $r>\left|a_{0}\right|$. For such $r$, consider any competitor $F$ in $(5.10)$, i.e., an $F \in C\left(\overline{\mathbb{D}}_{r} ; H^{1 / 2}\left(\mathbb{S}^{1} ; \mathbb{R}\right)\right)$ such that $F=\chi^{*}$ on $C_{r}$. Then we claim that there exists some $z_{0} \in \mathbb{D}_{r}$ (depending on $F$ ) such that the harmonic extension $u\left(\operatorname{tr} F\left(z_{0}\right)\right)$ of $\operatorname{tr} F\left(z_{0}\right)$ has a zero at $a_{0}$. Indeed from Theorem 4.1 and using the notation there, the map

$$
G: \overline{\mathbb{D}}_{r} \rightarrow \mathbb{D}, \quad \overline{\mathbb{D}}_{r} \ni z \stackrel{G}{\rightarrow} a(\operatorname{tr} F(z)) \in \mathbb{D}
$$

is continuous. Since $G$ coincides with the identity on $C_{r}, G$ must take the value $a_{0}$ in $\mathbb{D}_{r}$, by the Brouwer fixed point theorem.

Next, from Lemma 4.7 we find that $J^{*}\left(F\left(z_{0}\right)\right) \geq c\left(\beta, a_{0}\right)>\pi$, and thus $c^{*}>c\left(\beta, a_{0}\right)>\pi$. We obtain the desired conclusion by choosing $r$ sufficiently close to 1 in order to have $c_{1}^{*}<\min \left\{c\left(\beta, a_{0}\right), \pi+\delta_{0}\right\}$.

We now prove that $J^{*}$ is $C^{1}$ (Lemma 5.8). As an immediate consequence, we will characterize the Palais-Smale sequences associated to (5.10) (Corollary 5.12). Before proceeding, let us recall a useful elementary fact.

5.4 Lemma. Let $\mathscr{X}, \mathscr{Y}$ be normed spaces. Let $Z$ be a dense subspace of $\mathscr{X}$. Let $\mathscr{F} \in C(\mathscr{X} ; \mathscr{Y})$ and $\mathscr{T} \in C(\mathscr{X} ; B(\mathscr{X} ; \mathscr{Y}))$ be such that

$$
\frac{\partial \mathscr{F}}{\partial z}(x)=\mathscr{T}(x)(z), \quad \forall x, z \in \mathcal{Z}
$$

Then $\mathscr{F} \in C^{1}$ and (5.11) holds for every $x, z \in \mathscr{X}$.

Here, $B(\mathscr{X} ; \mathscr{Y})$ denotes the space of bounded linear operators from $\mathscr{X}$ into $\mathscr{Y}$. In practice, we will apply the above with $\mathcal{Z}=C^{\infty}$, and the point will be to check the continuity of $\mathscr{F}$ and $\mathscr{T}$.

We start with a straightforward consequence of the embedding $H^{1}(\mathbb{D}) \hookrightarrow L^{4}(\mathbb{D})$.

5.5 Lemma. The map $F_{\beta}$ is $C^{1}$ in $H^{1}(\mathbb{D} ; \mathbb{C})$ and

$$
F_{\beta}^{\prime}(u)(v)=\int_{\mathbb{D}} \nabla u \cdot \nabla v-\int_{\mathbb{D}} \beta u \cdot v\left(1-|u|^{2}\right), \quad \forall u, v \in H^{1}(\mathbb{D} ; \mathbb{C}) .
$$

Similarly, the map

$$
G: H^{1}(\mathbb{D} ; \mathbb{C}) \rightarrow \mathbb{R}, \quad H^{1}(\mathbb{D} ; \mathbb{C}) \ni u \mapsto \frac{1}{4} \int_{\mathbb{D}} \beta\left(1-|u|^{2}\right)^{2}
$$

is $C^{1}$, and $G^{\prime}(u)(v)=-\int_{\mathbb{D}} \beta u \cdot v\left(1-|u|^{2}\right)$.

Recall that - denotes the real scalar product.

5.6 Lemma. Assume that (5.8) holds. Then the map $g \rightarrow T(g)$, where $T(g)$ denotes the unique solution of (5.9), is $C^{1}$ in a neighborhood of $\mathscr{H}$. 
Proof. Consider the maps

$$
U: H^{1 / 2}\left(\mathbb{S}^{1} ; \mathbb{C}\right) \rightarrow H^{1}(\mathbb{D} ; \mathbb{C}), \quad g \stackrel{U}{\rightarrow} u(g)
$$

and

$$
V: H_{0}^{1}(\mathbb{D} ; \mathbb{C}) \times H^{1 / 2}\left(\mathbb{S}^{1} ; \mathbb{C}\right) \rightarrow H^{-1}(\mathbb{D} ; \mathbb{C}), \quad(v, g) \stackrel{V}{\rightarrow}-\Delta v-\beta(v+u(g))\left(1-|v+u(g)|^{2}\right) .
$$

Thanks to the embedding $H^{1}(\mathbb{D}) \hookrightarrow L^{4}(\mathbb{D})$ and to the continuity of $U$, it is easy to see that $V \in C^{1}$ and that the partial differential of $V(v, g)$ in the $v$ variable is given by

$$
\frac{\partial V}{\partial v}(v, g)(w)=-\Delta w-\beta\left(1-|v+u(g)|^{2}\right) w+2 \beta(w \cdot(v+u(g)))(v+u(g)), \quad \forall w \in H_{0}^{1}(\mathbb{D} ; \mathbb{C}) .
$$

The conclusion of the lemma follows via the implicit function theorem if we prove that the operator $W=\frac{\partial V}{\partial v}(v, g)$ is invertible at each couple $(T(g)-u(g), g)$ with $g \in \mathscr{H}$. Since $W$ is symmetric in $H_{0}^{1}(\mathbb{D} ; \mathbb{C}),{ }^{26}$ it suffices to prove that the quadratic form $Q$ associated to $W$ is definite positive. We note that

$$
Q(w)=\int_{\mathbb{D}}|\nabla w|^{2}-\int_{\mathbb{D}} \beta\left(1-|T(g)|^{2}\right)|w|^{2}+2 \int_{\mathbb{D}} \beta(w \cdot T(g))^{2} \geq \int_{\mathbb{D}}|\nabla w|^{2}-\int_{\mathbb{D}} \beta\left(1-|T(g)|^{2}\right)|w|^{2},
$$

and positivity follows from (5.8) combined with the maximum principle Lemma 2.17.

The next result is reminiscent of the fact that $H^{1}$ harmonic functions have traces of the normal derivative on the boundary.

5.7 Lemma. Let $g \in \mathscr{H}$ and let $u$ satisfy (5.9). Then the vector field $u \wedge \nabla u$ has a trace $\operatorname{tr}(u \wedge \nabla u)$ on $\mathbb{S}^{1}$, and this trace belongs to $H^{-1 / 2}\left(\mathbb{S}^{1}\right)$.

If, in addition, (5.8) holds, then the map

$$
Y: \not{H} \rightarrow H^{-1 / 2}\left(\mathbb{S}^{1}\right), \quad \mathscr{H} \ni g \stackrel{Y}{\rightarrow} \operatorname{tr}(T(g) \wedge \nabla T(g)) \in H^{-1 / 2}\left(\mathbb{S}^{1}\right),
$$

is continuous.

Proof. The vector field $u \wedge \nabla u$ belongs to $L^{2}$ and (by (5.9)) is divergence free. Existence of the trace is then standard; let us briefly recall the argument. By the ( $L^{2}$-version of the) Poincare lemma, we may write $u \wedge \nabla u=-\nabla^{\perp} h$ for some $h \in H^{1}(\mathbb{D})$. We then set

$$
\operatorname{tr}\left(u \wedge \frac{\partial u}{\partial v}\right)=\frac{\partial}{\partial \tau} \operatorname{tr} h \in H^{-1 / 2}\left(\mathbb{S}^{1}\right) .
$$

When $u$ is smooth, say $u \in C^{1}, \frac{\partial}{\partial \tau} \operatorname{tr} h$ is nothing else than $u \wedge \frac{\partial u}{\partial v}$; with an abuse of notation, we keep the notation $u \wedge \frac{\partial u}{\partial v}$ even if $u$ is merely $H^{1}$.

We note the integration by parts formula

$$
\int_{\mathbb{D}}(u \wedge \nabla u) \cdot \nabla \zeta=\left\langle u \wedge \frac{\partial u}{\partial v}, \operatorname{tr} \zeta\right\rangle_{H^{-1 / 2}, H^{1 / 2}}=\int_{\mathbb{S}^{1}}\left(u \wedge \frac{\partial u}{\partial v}\right) \zeta, \quad \forall \zeta \in H^{1}(\mathbb{D} ; \mathbb{R}) .
$$

\footnotetext{
${ }^{26}$ Here, we endow $\mathbb{C}$ with the scalar product of $\mathbb{R}^{2}$ and we identify $H_{0}^{1}(\mathbb{D} ; \mathbb{C})$ with a space of $\mathbb{R}^{2}$-valued functions.
} 
Although the last integral in (5.13) is defined only when $u$ is sufficiently smooth, we will use the integral notation for a general $u$.

The second part of the lemma is obtained as follows: let $g_{n} \rightarrow g$ in $\mathscr{H}$. Set $u_{n}=T\left(g_{n}\right)$ and $u=T(g)$. Then $u_{n} \rightarrow u$ in $H^{1}$ (since $T$ is continuous). Using the fact that $\left|u_{n}\right| \leq 1$ (Lemma 2.17), we find that $u_{n} \wedge \nabla u_{n} \rightarrow u \wedge \nabla u$ in $L^{2}$. If we normalize the corresponding potentials $h_{n}$ such that $\int_{\mathbb{D}} h_{n}=0$, then $h_{n} \rightarrow h$ in $H^{1}$. This implies convergence of $\operatorname{tr}\left(u_{n} \wedge \nabla u_{n}\right)$ to $\operatorname{tr}(u \wedge \nabla u)$ in $H^{-1 / 2}$.

5.8 Lemma. Assume (5.8). Then we have $J^{*} \in C^{1}$ and, if $u:=T\left(N_{0} e^{\imath \psi}\right)$, then

$$
J^{* \prime}(\psi)(\eta)=\int_{\mathbb{S}^{1}}\left(u \wedge \frac{\partial u}{\partial v}\right) \eta=\int_{\mathbb{D}}(u \wedge \nabla u) \cdot \nabla \zeta, \forall \psi, \eta \in H^{1 / 2}\left(\mathbb{S}^{1} ; \mathbb{R}\right), \forall \zeta \in H^{1}(\mathbb{D} ; \mathbb{R}) \text { such that } \operatorname{tr} \zeta=\eta .
$$

More generally, the same holds if we consider the map

$$
H^{1 / 2}\left(\mathbb{S}^{1} ; \mathbb{R}\right) \ni \psi \stackrel{J_{d}^{*}}{\longrightarrow} F_{\beta}\left(T\left(N_{0}^{d} e^{\imath \psi}\right)\right), \quad \text { where } d \in \mathbb{Z} \text {. }
$$

Proof. Note that the equality of the two integrals in (5.14) is a consequence of (5.13). In order to prove (5.14), we rely on Lemma 5.4. Clearly, $J^{*}$ is continuous. On the other hand, if we let $\zeta=u(\eta)$ in (5.14) and we use the continuity of the map $H^{1 / 2} \ni \psi \mapsto u \wedge \nabla u \in L^{2}$, then we see that the second integral in (5.14) defines a map

$$
\mathscr{T} \in C\left(H^{1 / 2}\left(\mathbb{S}^{1} ; \mathbb{R}\right), H^{-1 / 2}\left(\mathbb{S}^{1} ; \mathbb{R}\right)\right), \quad \mathscr{T}(\psi)(\eta):=\int_{\mathbb{D}}(u \wedge \nabla u) \cdot \nabla u(\eta) .
$$

Therefore, it suffices to prove that (5.14) holds when $\psi$ and $\eta$ are smooth and when $\zeta=u(\eta)$. For such $\psi$ and $\eta$, let $g_{t}=N_{0} e^{l(\psi+t \eta)}$ and set $u_{t}=T\left(g_{t}\right)$ (so that $u=u_{0}$ ). It is easy to see that $t \mapsto g_{t} \in \mathscr{H}$ is smooth. By Lemma 5.5 and Lemma 5.6, the maps $t \mapsto u_{t}$ and $t \mapsto J^{*}(\psi+t \eta)$ are $C^{1}$, and

$$
\frac{d}{d t}\left[J^{*}(\psi+t \eta)\right]=\int_{\mathbb{D}} \nabla u_{t} \cdot \nabla\left(\frac{d}{d t} u_{t}\right)-\int_{\mathbb{D}} \beta u_{t} \cdot\left(\frac{d}{d t} u_{t}\right)\left(1-\left|u_{t}\right|^{2}\right) .
$$

On the other hand, if $v \in H^{1}(\Omega ; \mathbb{C})$ is such that $v=\imath u \zeta$ on $\mathbb{S}^{1}$, where $\zeta \in C^{1}\left(\mathbb{S}^{1} ; \mathbb{R}\right)$, and if $u$ solves (5.9), then

$$
\int_{\mathbb{D}} \nabla u \cdot \nabla v-\int_{\mathbb{D}} \beta(u \cdot v)\left(1-|u|^{2}\right)=\int_{\mathbb{S}^{1}}\left(u \wedge \frac{\partial u}{\partial v}\right) \zeta
$$

Indeed, when $u$ is smooth, this is a consequence of (5.9) and of the identity

$$
\frac{\partial u}{\partial v} \cdot(\imath u \zeta)=\left(u \wedge \frac{\partial u}{\partial v}\right) \zeta \quad \text { on } \mathbb{S}^{1} .
$$

The general case follows by approximation, using Lemma 2.11 . and the following fact: given $\beta$ satisfying (5.8), we may find a uniformly bounded sequence $\left(\beta_{n}\right)$ of smooth function satisfying (5.8) and such that $\beta_{n} \rightarrow \beta .^{27}$

We next note that $u_{t}=N_{0} e^{\imath(\psi+t \eta)}$ on $\mathbb{S}^{1}$, and therefore $\frac{\partial}{\partial t} u_{t}=\imath u_{t} \eta$ on $\mathbb{S}^{1}$. Thus, in (5.16), we may take $u=u_{t}$ and $v=\frac{\partial}{\partial t} u_{t}$. We obtained the first identity in (5.14) by combining this remark with (5.15).

\footnotetext{
${ }^{27}$ For an alternative argument not relying on (5.8), see Remark 5.9 below.
} 
5.9 Remark. Here is an alternative proof of (5.16), valid without the assumption (5.8). In view of (5.9), (5.16) holds when $v \in H_{0}^{1}$, so that it suffices to prove (5.16) for a special $v$ such that $v=\imath u \zeta$ on $\mathbb{S}^{1}$. Consider a $C^{1}$ extension of $\zeta$, still denoted $\zeta$, and let $v=\imath u \zeta$. For this $v,(5.16)$ is nothing else but (5.13).

5.10 Remark. Lemma 5.8 hides a small miracle. Recall that $J^{*}$ is constructed as follows:

$$
J^{*}=F_{\beta} \circ T \circ S, \quad H^{1 / 2}\left(\mathbb{S}^{1} ; \mathbb{R}\right) \ni \psi \stackrel{S}{\rightarrow} g=N_{0} e^{\imath \psi} \stackrel{T}{\rightarrow} u=T(g) \stackrel{F_{\beta}}{\rightarrow} F_{\beta}(u) .
$$

By Lemmas 5.6 and 5.5, we know that $T$ and $F_{\beta}$ are $C^{1}$. We also know that $J^{*}$ is $C^{1}$ (Lemma 5.8). However, $S$ is not $C^{1}$; see Lemma 5.11 below. Since $J^{*}$ is the composition of two operators, one, $F_{\beta} \circ T$, smooth, the other one, $S$, non smooth, the conclusion of Lemma 5.8 is that the smoothing effect prevails. Smoothness comes from the main ingredient of the proof of Lemma 5.8, which is the existence of the boundary trace of the vector field $u \wedge \nabla u$ whenever $u$ is a solution of (5.9). In turn, this relies basically on the Poincaré lemma and on the maximum principle. All in all, this turns $F_{\beta} \circ T$ (and finally $J^{*}$ ) into a smooth operator. On the other hand, we note that $S$ is almost smooth: it is clearly Lipschitz.

5.11 Lemma. $S$ is not differentiable. More generally, if $g \in \mathscr{H}$, then the map

$$
S_{g}: H^{1 / 2}\left(\mathbb{S}^{1} ; \mathbb{R}\right) \rightarrow \mathscr{H}, \quad H^{1 / 2}\left(\mathbb{S}^{1} ; \mathbb{R}\right) \ni \psi \stackrel{S_{g}}{\longrightarrow} g e^{\imath \psi} \in \mathscr{H}
$$

is not differentiable.

Proof. We start by reducing the general case to the special case $g=1$. Let $d=\operatorname{deg} g$ and write $g=N_{0}^{d} e^{\imath \varphi}$, with $\varphi \in H^{1 / 2}\left(\mathbb{S}^{1} ; \mathbb{R}\right)\left(c f\right.$ Lemma 2.64 .). Multiplication with $N_{0}^{d}$ being clearly a linear continuous bijective operator in $H^{1 / 2}$, we see that $S_{g}$ is differentiable at $\psi$ if and only if $S$ is differentiable at $\psi+\varphi$.

We continue by finding functions $\psi$ such that $S$ is not differentiable at $\psi$. It is easy to see that, for $\eta \in C^{\infty}\left(\mathbb{S}^{1} ; \mathbb{R}\right)$, we have

$$
\lim _{t \rightarrow 0} \frac{S(\psi+t \eta)-S(\psi)}{t}=A(\psi, \eta):=\imath S(\psi) \eta
$$

the limit being considered in $H^{1 / 2}$. If $S$ is differentiable at $\psi$, then (5.17) holds for every $\eta \in H^{1 / 2}\left(\mathbb{S}^{1} ; \mathbb{R}\right)$, and the map $A(\psi, \eta)$ belongs to $H^{1 / 2}$. The heuristics for concluding is the following: $H^{1 / 2} \cap L^{\infty}$ is an algebra, but $H^{1 / 2}$ is not. Thus, if we pick a function $\eta \in H^{1 / 2} \cap L^{\infty}$, then $A(\psi, \eta) \in H^{1 / 2}$, but this need not hold when $\eta$ is merely $H^{1 / 2}$. Formally, we continue as follows: if $S$ is differentiable at $\psi$, then $A(\psi, \psi)=\imath e^{\imath \psi} \psi \in H^{1 / 2}$, and in particular $B(\psi):=\psi \cos \psi$ belongs to $H^{1 / 2}$. We recall the following result of Bourdaud and Kateb [13]: a superposition operator $\psi \mapsto G \circ \psi$ acts on $H^{1 / 2}$ if and only if $G$ is Lipschitz. Though the result in [13] is stated in $H^{1 / 2}\left(\mathbb{R}^{n}\right)$, the construction of counterexamples yields, for a non Lipschitz $G$, compactly supported maps $\psi$ such that $G \circ \psi \notin H^{1 / 2}$; see [38, Section 5.3.1, proof of Theorem 1]. Thus, even on the circle, $G$ has to be Lipschitz. We conclude by noting that $t \mapsto t \cos t$ is not Lipschitz.

We are now in position to construct sequences of almost critical points. By combining Theorem 5.1 with Lemmas 5.3 and 5.8, we obtain the following. 
5.12 Corollary. Assume that (5.8) holds. Let $c^{*}$ be defined by (5.10). Then, for each $\delta>0$, there exists some $\psi_{\delta} \in H^{1 / 2}\left(\mathbb{S}^{1} ; \mathbb{R}\right)$ such that $u_{\delta}=T\left(N_{0} e^{\imath \psi_{\delta}}\right)$ satisfies

$$
c^{*}-\delta \leq J^{*}\left(\psi_{\delta}\right)=F_{\beta}\left(u_{\delta}\right) \leq c^{*}+\delta
$$

and

$$
\left|\int_{\mathbb{S}^{1}}\left(u_{\delta} \wedge \frac{\partial u_{\delta}}{\partial v}\right) \eta\right| \leq \sqrt{\delta}|\eta|_{H^{1 / 2}}, \quad \forall \eta \in H^{1 / 2}\left(\mathbb{S}^{1} ; \mathbb{R}\right)
$$

In particular, the corollary applies if we take a large $\varepsilon$ in the original energy $E_{\varepsilon}$. Note that, by (5.13), (5.19) is equivalent to

$$
\left|\int_{\mathbb{D}}\left(u_{\delta} \wedge \nabla u_{\delta}\right) \cdot \nabla \zeta\right| \leq \sqrt{\delta}\|\nabla \zeta\|_{L^{2}}, \quad \forall \zeta \in H^{1}(\mathbb{D} ; \mathbb{R}) .
$$

5.13 Corollary. Assume that (5.8) holds. Then every weak limit of $u_{\delta}$ as $\delta \rightarrow 0$ is a critical point of $F_{\beta}$ in $\mathscr{G}$, i.e., a solution of (2.23).

Proof. Assume that (possibly along a subsequence) $u_{\delta} \rightarrow u$ in $H^{1}$. Then on the one hand $u$ satisfies $-\Delta u=\beta u\left(1-|u|^{2}\right)$ (this relies on the maximum principle Lemma 2.17). On the other hand, $u_{\delta} \wedge \nabla u_{\delta} \rightarrow$ $u \wedge \nabla u$ in $L^{2}$, and thus (using (5.20)) $u$ satisfies (2.21), which is equivalent to (2.23), by Lemma 2.15 .

\section{Existence of critical points}

We establish here the following generalization of Theorem 1.1.

6.1 Theorem. Assume that (5.8) holds and that the value $c^{*}$ in (5.10) satisfies

$$
c^{*}<2 \pi
$$

Then $c^{*}$ is a critical value of $F_{\beta}$ in $\mathscr{G}_{1}$, i.e., there exists a solution $u$ of (2.23) with $d=1$ such that $F_{\beta}(u)=c^{*}$.

Before proceeding to the proof, let us note that, with the choice $F(a)=N_{a}, a \in \overline{\mathbb{D}}_{r}$, in (5.10), and for $\beta=\frac{1}{\varepsilon^{2}} \mathrm{Jac} \Phi^{-1}$ in $(2.18)$, where $\Phi: \Omega \rightarrow \mathbb{D}$ is a conformal representation, we find that

$$
c^{*} \leq \max _{a \in \mathbb{D}}\left(\int_{\mathbb{D}}\left|\nabla M_{a}\right|^{2}+\frac{1}{4 \varepsilon^{2}} \int_{\mathbb{D}}\left(1-\left|M_{a}\right|^{2}\right)^{2} \operatorname{Jac} \Phi^{-1}\right) \leq \pi+\frac{|\Omega|}{4 \varepsilon^{2}} .
$$

The latter quantity is $<2 \pi$ for large $\varepsilon$. Thus Theorem 6.1 generalizes indeed Theorem 1.1.

Proof. Let $u_{\delta}$ be as in Corollary 5.12. Let $u$ be such that, up to a subsequence $\delta_{n} \rightarrow 0$, we have $u_{\delta_{n}} \rightarrow u$ in $H^{1}$. By Corollary 5.13, $u$ satisfies (2.23). In order to complete the proof of Theorem 6.1, we will prove that $\operatorname{deg}\left(u, \mathbb{S}^{1}\right)=1$ and that $F_{\beta}(u)=c^{*}$. In order to prove the former assertion, it suffices to obtain the existence of some $r \in(0,1)$ and of some $\lambda>0$ such that (possibly along a further subsequence, still denoted $\left.\left(\delta_{n}\right)\right)$

$$
\left|u_{\delta_{n}}(z)\right| \geq \lambda \quad \text { when }|z| \geq r
$$


Indeed, assume for a moment that (6.2) holds. Then deg $\left(u, \mathbb{S}^{1}\right)=1$, by Lemma 2.10. Also, condition (6.2) is essential in the proof of the strong convergence $u_{\delta_{n}} \rightarrow u$ in $H^{1}$.

Validity of (6.2) is established by contradiction: assuming that there exist sequences $\delta_{n} \rightarrow 0$ and $a_{n}$ such that

$$
u_{\delta_{n}}\left(a_{n}\right) \rightarrow 0 \text { and }\left|a_{n}\right| \rightarrow 1,
$$

we will prove that

$$
F_{\beta}\left(u_{\delta_{n}}\right) \rightarrow \pi
$$

which contradicts Lemma 5.3.

Before proceeding to the proof, we introduce lighter notation: we write $u_{n}$ instead of $u_{\delta_{n}}$ and still denote by $\left(u_{n}\right)$ any subsequence extracted from $\left(u_{n}\right)$, and the same for $\left(a_{n}\right)$.

We start by rescaling the sequence $\left(u_{n}\right)$ : we let

$$
\Phi_{n}=M_{-a_{n}}, v_{n}=u \circ \Phi_{n}, \beta_{n}=\beta \circ \Phi_{n} \operatorname{Jac} \Phi_{n}, F_{n}(w)=\frac{1}{2} \int_{\mathbb{D}}|\nabla w|^{2}+\frac{1}{4} \int_{\mathbb{D}} \beta_{n}\left(1-|w|^{2}\right)^{2} .
$$

In particular, using Corollary 5.12 and the conformal invariance of the Dirichlet integral, we find that the rescaled sequence $\left(v_{n}\right) \subset \mathscr{G}_{1}$ satisfies, with $c_{n} \rightarrow 0$,

$$
\begin{cases}-\Delta v_{n}=\beta_{n} v_{n}\left(1-\left|v_{n}\right|^{2}\right) & \text { in } \mathbb{D} \\ \left|\operatorname{tr} v_{n}\right|=1 & \\ \left|\int_{\mathbb{D}}\left(v_{n} \wedge \nabla v_{n}\right) \cdot \nabla \zeta\right| \leq c_{n}\|\nabla \zeta\|_{L^{2}}, \quad \forall \zeta \in H^{1}(\mathbb{D}) . \\ v_{n}(0) \rightarrow 0 \\ F_{n}\left(v_{n}\right)=F_{\beta}\left(u_{n}\right) \rightarrow c^{*}\end{cases}
$$

We start by collecting some straightforward properties of $\beta_{n}$ and $v_{n}$.

6.2 Lemma. 1. $\beta_{n} \rightarrow 0$ uniformly on compact subsets. In particular, $\left(v_{n}\right)$ converges, up to a subsequence and in $C_{l o c}^{1, \alpha}(\mathbb{D}), 0<\alpha<1$, to some solution $v \in \mathscr{G}$ of $(2.23)$ with $\beta=0$ and such that $v(0)=0$. Moreover, (by Lemma 3.5) $v \in \mathscr{G}_{d}$ for some $d \neq 0$, and $v$ is either a d-Blaschke product, or the conjugate of such a product.

2. $\lambda_{1}\left(-\Delta-\beta_{n}\right)>0$. In particular, $v_{n}$ is a minimizer of $F_{\beta_{n}}$ with respect with its own boundary condition.

Proof. The fact that $\beta_{n} \rightarrow 0$ uniformly on compact subsets followed from the definition (6.5) combined with the fact that $\left|\nabla \Phi_{n}\right| \rightarrow 0$ uniformly on compact subsets. This convergence, together with the equation (6.6), implies that $\Delta v_{n} \rightarrow 0$ uniformly on compact subsets. Let $K$ be a compact subset of $\mathbb{D}$ and let $L \subset \mathbb{D}$ be a compact neighborhood of $K$. By standard elliptic estimates [27, Theorem 9.13, p. 239], we have

$$
\left\|v_{n}\right\|_{W^{2, p}(K)} \leq C\left(\left\|v_{n}\right\|_{L^{2}(\mathbb{D})}+\left\|\Delta v_{n}\right\|_{L^{p}(L)}\right) \leq C^{\prime}, \quad 1<p<\infty,
$$

and thus $\left(v_{n}\right)$ is bounded in $W_{l o c}^{2, p}(\mathbb{D}), 1<p<\infty$. By the Sobolev embeddings, $\left(v_{n}\right)$ is bounded in $C_{l o c}^{1, \alpha}(\mathbb{D}), 0<\alpha<1$. Using (6.6) and Ascoli's theorem, we find that (up to a subsequence) $\left(v_{n}\right)$ converges in $C_{\text {loc }}^{1, \alpha}(\mathbb{D}), 0<\alpha<1$, to some harmonic function $v \in \mathscr{G}$ such that $v(0)=0$. The last part in item 1 . is simply a restatement of Lemma 3.5 .

Item 2. follows from (5.8). 
Step 1 (in the proof of Theorem 6.1). Let $v$ be as in Lemma 6.2. Then we claim that $v=M_{\alpha, 0}$ for some $\alpha \in \mathbb{S}^{1}$.

Indeed, we adapt the argument leading to (4.1): we have, by the Price Lemma 2.18 and Corollary 3.2,

$$
2 \pi>c^{*} \geq \liminf \frac{1}{2} \int_{\mathbb{D}}\left|\nabla v_{n}\right|^{2} \geq \frac{1}{2} \int_{\mathbb{D}}|\nabla v|^{2}+\pi|1-d| \geq \pi|d|+\pi|1-d|,
$$

where $d=\operatorname{deg}\left(v, \mathbb{S}^{1}\right)$. We find that $d=0$ or $d=1$. Since $d \neq 0$ (by Lemma 6.21 .), we obtain the desired conclusion by combining Lemma 6.21 . with the fact that $v(0)=0$.

Step 2. There exist $r \in(0,1)$ and $\lambda>0$ such that $\left|v_{n}(z)\right| \geq \lambda$ when $|z| \geq r$.

In order to prove this, we argue by contradiction and assume that up to a subsequence we have $v_{n}\left(z_{n}\right) \rightarrow 0$, where the points $z_{n} \in \mathbb{D}$ are such that $\left|z_{n}\right| \rightarrow 1$. By repeating the arguments leading to (4.10) and (4.11), we obtain that, for each $s \in(0,1)$, there exists some $R \in(0,1)$ such that

$$
\liminf \frac{1}{2} \int_{\mathbb{D}_{R}}\left|\nabla v_{n}\right|^{2} \geq \pi s^{2}
$$

and

$$
\liminf \frac{1}{2} \int_{\mathbb{D}_{\mathbb{D}_{R}}}\left|\nabla v_{n}\right|^{2} \geq \pi s^{2}
$$

Indeed, (6.7) is obtained exactly as (4.10). On the other hand, if we want to reproduce the argument leading to (4.11) we have to be in position to apply Lemma 4.2. This lemma requires that $v_{n}$ minimizes $F_{n}$ with respect to its own boundary conditions. This holds indeed in our case, by Lemma 6.2 2.

By combining (6.7) with (6.8), we find that $\liminf \frac{1}{2} \int_{\mathbb{D}}\left|\nabla v_{n}\right|^{2} \geq 2 \pi$. But this contradicts the assumption $c^{*}<2 \pi$.

Step 3. We have (the contradiction) $F_{n}\left(v_{n}\right) \rightarrow \pi$.

This step is a consequence of Step 2., of (6.6) and of Lemma 6.3 below.

We start by stating the technical assumptions required in Lemma 6.3. For simplicity, we will state Lemma 6.3 when the underlying domain is $\mathbb{D}$, but the same argument works for any simply connected domain.

We consider two sequences $\left(\beta_{n}\right)$ and $\left(v_{n}\right)$ and two maps $\gamma$ and $v$ such that:

$$
\begin{aligned}
& \beta_{n}, \gamma \in L^{\infty}(\mathbb{D}), \beta_{n}, \gamma \geq 0, \\
& \beta_{n} \rightarrow \gamma \text { uniformly on compact subsets of } \mathbb{D}, \\
& v_{n}, v \in H^{1}(\mathbb{D} ; \mathbb{C}),\left|\operatorname{tr} v_{n}\right|=1, v_{n} \rightarrow v \text { in } H^{1}, \\
& -\Delta v_{n}=\beta_{n} v_{n}\left(1-\left|v_{n}\right|^{2}\right) \text { in } \mathbb{D}, \\
& \left|\int_{\mathbb{D}}\left(v_{n} \wedge \nabla v_{n}\right) \cdot \nabla \zeta\right| \leq c_{n}\|\nabla \zeta\|_{L^{2}}, \forall \zeta \in H^{1}(\mathbb{D}), \text { with } c_{n} \rightarrow 0 \text { as } n \rightarrow \infty .
\end{aligned}
$$

Unlike our next hypothesis (6.14), the above assumptions are naturally satisfied by sequences of almost critical points obtained via the functional $J^{*}$ (as in Corollary 5.12).

6.3 Lemma. Assume that (6.9)-(6.13) hold. Assume in addition that there exists some $\lambda>0$ such that

$$
\left|v_{n}(z)\right| \geq \lambda \quad \forall z \in \mathbb{D} \text { such that }|z| \geq 1-\lambda .
$$

Then we have

$$
\int_{\mathbb{D}}\left|\nabla v_{n}\right|^{2} \rightarrow \int_{\mathbb{D}}|\nabla v|^{2}
$$


and

$$
\int_{\mathbb{D}} \beta_{n}\left(1-\left|v_{n}\right|^{2}\right)^{2} \rightarrow \int_{\mathbb{D}} \gamma\left(1-|v|^{2}\right)^{2}
$$

Equivalently, we have $v_{n} \rightarrow v$ strongly in $H^{1}$ and

$$
F_{n}\left(v_{n}\right) \rightarrow F_{\gamma}(v)
$$

Step 3 completed. We take, in the above lemma, $\gamma=0$ and $v=M_{\alpha, 0}$ as in Step 1.

Proof of Lemma 6.3. It suffices to establish (6.15)-(6.16) along a subsequence.

By the maximum principle Lemma 2.17, we have $\left|v_{n}\right|,|v| \leq 1$ in $\mathbb{D}$. By standard elliptic estimates [27, Theorem 9.13, p. 239], we have

$$
v_{n} \rightarrow v \text { in } W_{l o c}^{2, p}(\mathbb{D}), \forall p<\infty .
$$

By (6.10) and (6.18), we find that (6.15) and (6.16) hold if we replace $\mathbb{D}$ by $\mathbb{D}_{1-\varepsilon}$ for each $\varepsilon>0$. On the other hand, we have

$$
\lim _{\varepsilon \rightarrow 0} \int_{\mathbb{D} \backslash \overline{\mathbb{D}}_{1-\varepsilon}}\left(|\nabla v|^{2}+\gamma\left(1-|v|^{2}\right)^{2}\right)=0
$$

Therefore, it suffices to prove that

$$
\lim _{\varepsilon \rightarrow 0} \limsup _{n \rightarrow \infty} \int_{\mathbb{D} \backslash \overline{\mathbb{D}}_{1-\varepsilon}}\left(\left|\nabla v_{n}\right|^{2}+\beta_{n}\left(1-\left|v_{n}\right|^{2}\right)^{2}\right)=0 .
$$

By (6.18), $v_{n} \rightarrow v$ uniformly on compact subsets of $\mathbb{D}$. Combining this fact with (6.14), we find that for large $n$ we have

$$
d:=\operatorname{deg}\left(\frac{v}{|v|}, C_{r}\right)=\operatorname{deg}\left(\frac{v_{n}}{\left|v_{n}\right|}, C_{r}\right), \forall r \in[1-\lambda, 1]
$$

By Corollary 2.9 applied with $u^{1}=\left(\frac{z}{|z|}\right)^{d}, u^{2}=v_{n}, \omega=\mathbb{D} \backslash \overline{\mathbb{D}}_{1-\lambda}$, we may write, in $\omega, v_{n}=\rho_{n} e^{\imath\left(d \theta+\varphi_{n}\right)}$, and similarly $v=\rho e^{\imath(d \theta+\varphi)}$, with $\lambda \leq \rho_{n}, \rho \leq 1$, and $\varphi_{n}, \varphi \in H^{1}(\omega)$. By (2.11), we find that, possibly after extracting suitable multiples of $2 \pi$, we have $\varphi_{n} \rightarrow \varphi$ and $\rho_{n} \rightarrow \rho$ in $H^{1}(\omega)$. On the other hand, by (6.18), we have $\varphi_{n} \rightarrow \varphi$ and $\rho_{n} \rightarrow \rho$ in $C_{l o c}^{1}(\omega)$. We also note the fact that $v \in C^{1}(\overline{\mathbb{D}})$, by Lemma 2.15.

We next translate the properties of $v_{n}$ in terms of $\rho_{n}$ and $\varphi_{n}:(6.12)$ and (6.13) imply that

$$
\left\{\begin{array}{ll}
\operatorname{div}\left(\rho_{n}^{2} \nabla\left(d \theta+\varphi_{n}\right)\right)=0 & \text { in } \omega \\
-\Delta \rho_{n}=\beta_{n} \rho_{n}\left(1-\rho_{n}^{2}\right)-\rho_{n}\left|\nabla\left(d \theta+\varphi_{n}\right)\right|^{2} & \text { in } \omega \\
\operatorname{tr} \rho_{n}=1 & \text { on } \mathbb{S}^{1} \\
v_{n} \wedge \nabla v_{n}=\rho_{n}^{2} \nabla\left(d \theta+\varphi_{n}\right) & \text { in } \omega \\
\left|\int_{\mathbb{D}}\left(v_{n} \wedge \nabla v_{n}\right) \cdot \nabla \zeta\right| \leq c_{n}\|\nabla \zeta\|_{L^{2}}, & \forall \zeta \in H^{1}(\mathbb{D})
\end{array} .\right.
$$

Let $0<\varepsilon<\lambda$. Since $\varphi_{n} \rightarrow \varphi$ in $C^{1}\left(C_{1-\varepsilon}\right)$, we find that that the function $\varphi_{n}-\varphi$, defined in $\mathbb{D} \backslash \overline{\mathbb{D}}_{1-\varepsilon}$, has an extension $\zeta_{n} \in H^{1}(\mathbb{D})$ such that $\left\|\nabla \zeta_{n}\right\|_{L^{2}\left(\mathbb{D}_{1-\varepsilon}\right)} \rightarrow 0$. Using the fact that

$$
\rho_{n}^{2} \nabla(d \theta) \rightarrow \rho^{2} \nabla(d \theta) \text { and } \rho_{n}^{2} \nabla \varphi_{n} \rightarrow \rho^{2} \nabla \varphi \quad \text { in } L^{2}(\omega)
$$


we find that

$$
\begin{aligned}
0 & =\lim _{n \rightarrow \infty} \int_{\mathbb{D}}\left(v_{n} \wedge \nabla v_{n}\right) \cdot \nabla \zeta_{n}=\lim _{n \rightarrow \infty} \int_{\mathbb{D} \backslash \overline{\mathbb{D}}_{1-\varepsilon}}\left(v_{n} \wedge \nabla v_{n}\right) \cdot \nabla \zeta_{n} \\
& =\lim _{n \rightarrow \infty} \int_{\mathbb{D} \backslash \overline{\mathbb{D}}_{1-\varepsilon}}\left[\rho_{n}^{2} \nabla\left(d \theta+\varphi_{n}\right)\right] \cdot \nabla\left(\varphi_{n}-\varphi\right)=\lim _{n \rightarrow \infty} \int_{\mathbb{D} \backslash \overline{\mathbb{D}}_{1-\varepsilon}} \rho_{n}^{2}\left|\nabla \varphi_{n}\right|^{2}-\int_{\mathbb{D}_{\mathbb{\mathbb { D }}_{1-\varepsilon}}} \rho^{2}|\nabla \varphi|^{2},
\end{aligned}
$$

which implies easily that

$$
\lim _{n \rightarrow \infty} \int_{\mathbb{D}_{\mathbb{D}} \overline{\mathbb{D}}_{1-\varepsilon}} \rho_{n}^{2}\left|\nabla\left(d \theta+\varphi_{n}\right)\right|^{2}=\int_{\mathbb{D}_{\mathbb{D}_{1-\varepsilon}}} \rho^{2}|\nabla(d \theta+\varphi)|^{2},
$$

and in particular

$$
\lim _{\varepsilon \rightarrow 0} \lim _{n \rightarrow \infty} \int_{\mathbb{D} \backslash \overline{\mathbb{D}}_{1-\varepsilon}} \rho_{n}^{2}\left|\nabla\left(d \theta+\varphi_{n}\right)\right|^{2}=0 .
$$

We next multiply by $\eta_{n}=1-\rho_{n}$ the equation satisfied by $\rho_{n}$ and find that

$$
\int_{\mathbb{D} \backslash \overline{\mathbb{D}}_{1-\varepsilon}}\left(\left|\nabla \rho_{n}\right|^{2}+\frac{\rho_{n}}{1+\rho_{n}} \beta_{n}\left(1-\left|v_{n}\right|^{2}\right)^{2}\right)=\int_{\mathbb{D}_{\mathbb{D}_{1-\varepsilon}}} \rho_{n} \eta_{n}\left|\nabla\left(d \theta+\varphi_{n}\right)\right|^{2}+\int_{C_{1-\varepsilon}} \eta_{n} \frac{\partial \rho_{n}}{\partial v},
$$

$v$ being the normal exterior to $\mathbb{D}_{1-\varepsilon}$.

We next note that, since $v \in C^{1}(\overline{\mathbb{D}})$ and $|v|=1$ on $\mathbb{S}^{1}$, we have

$$
\lim _{\varepsilon \rightarrow 0} \lim _{n \rightarrow \infty} \int_{C_{1-\varepsilon}} \eta_{n} \frac{\partial \rho_{n}}{\partial v}=\lim _{\varepsilon \rightarrow 0} \int_{C_{1-\varepsilon}}(1-\rho) \frac{\partial \rho}{\partial v}=0 .
$$

By combining (6.22) with (6.21), with(6.23), and with the assumption (6.14), we find that

$$
\lim _{\varepsilon \rightarrow 0} \limsup _{n \rightarrow \infty} \int_{\mathbb{D} \backslash \overline{\mathbb{D}}_{1-\varepsilon}}\left(\left|\nabla \rho_{n}\right|^{2}+\beta_{n}\left(1-\left|v_{n}\right|^{2}\right)^{2}\right)=0
$$

We obtain (6.19) (and thus complete the proof of Lemma 6.3) by combining (6.21) with (6.24) and with the identity (2.11).

By combining (6.2) (whose validity has been established in the course of the proof of Theorem 6.1) with Lemma 6.3 applied to the original sequence $\left(u_{n}\right)$ and to the weights $\beta_{n}=\beta$ and $\gamma=\beta$, we obtain the following improvement of Theorem 6.1.

6.4 Theorem. Assume that (5.8) holds and that $c^{*}<2 \pi$. Then $J^{*}$ satisfies the Palais-Smale condition at level $c^{*}$.

\section{Asymptotic behavior of critical points as $\varepsilon \rightarrow \infty$}

We describe here the asymptotic behavior, when $\varepsilon \rightarrow \infty$, of critical points of $E_{\varepsilon}$ in $\mathscr{E}_{1}$ obtained by the mountain pass approach described in Section 5. For simplicity, we transfer the problem on $\mathbb{D}$ (as explained at the end of Section 2) and consider the energy $F_{\beta}$, where

$$
\beta=\beta_{\varepsilon}=\frac{1}{\varepsilon^{2}} \operatorname{Jac} \Phi^{-1}=\frac{1}{\varepsilon^{2}} w .
$$


In order to emphasize dependence on $\varepsilon$, we denote by $c_{\varepsilon}^{*}$ the number $c^{*}$ associated to $F_{\beta}$, i.e.,

$$
c_{\varepsilon}^{*}:=\inf \left\{\max _{\overline{\mathbb{D}}_{r}} F_{\beta_{\varepsilon}}\left(T\left(N_{0} e^{\imath F}\right)\right) ; F \in C\left(\overline{\mathbb{D}}_{r} ; H^{1 / 2}\left(\mathbb{S}^{1} ; \mathbb{R}\right)\right), F(a)=\psi_{a} \text { for } a \in C_{r}\right\}
$$

recall that

$$
J^{*}(\psi)=F_{\beta_{\varepsilon}}\left(T\left(N_{0} e^{\imath F}\right)\right) .
$$

We assume that $r$ is sufficiently close to 1 so that Lemma 5.3 holds (at this point we also allow $r$ to depend on $\varepsilon){ }^{28}$ Before stating and proving our main result in this section, let us introduce some useful notation. Given $v \in H^{1}(\mathbb{D} ; \mathbb{C})$,

$\widetilde{v}$ is the harmonic function in $\mathbb{D}$ which agrees with $v$ on $\mathbb{S}^{1}$, i.e., $\widetilde{v}=u(\operatorname{tr} v)$.

Also, given a function $F \in C\left(\overline{\mathbb{D}}_{r} ; H^{1 / 2}\left(\mathbb{S}^{1} ; \mathbb{R}\right)\right)$, we let

$$
\underline{F}(a):=\text { the harmonic extension of } N_{0} e^{\imath F(a)} .
$$

We next turn to the description of the asymptotic behavior of $u_{\varepsilon}$ as $\varepsilon \rightarrow \infty$. Clearly, the Moebius transforms $M_{\alpha, a}$ satisfy

$$
\int_{\mathbb{D}} w\left(1-\left|M_{\alpha, a}\right|^{2}\right)^{2}>0, \forall \alpha \in \mathbb{S}^{1}, \forall a \in \mathbb{D} \text {, and } \lim _{|a| \rightarrow 1} \int_{\mathbb{D}} w\left(1-\left|M_{\alpha, a}\right|^{2}\right)^{2}=0 .
$$

Therefore, the maximization problem

$$
M:=\max _{\substack{a \in \mathbb{D} \\ \alpha \in \mathbb{S}^{1}}} \int_{\mathbb{D}} w\left(1-\left|M_{\alpha, a}\right|^{2}\right)^{2}
$$

has a solution and, if $M_{\alpha, a}$ is a maximizer, then so is $M_{\gamma, a}$ for every $\gamma \in \mathbb{S}^{1}$. In addition, there exists some $r_{0}<1$ such that every maximizer in (7.6) satisfies $|a| \leq r_{0}$. In what follows, we always assume that $r_{0}<r<1$.

7.1 Theorem. Let $u_{\varepsilon}$ be obtained via (7.2). Then, possibly up to a subsequence, $u_{\varepsilon} \rightarrow M_{\alpha, a}$ as $\varepsilon \rightarrow \infty$, strongly in $H^{1}(\mathbb{D})$. Here, $M_{\alpha, a}$ is a maximizer in (7.6).

7.2 Remark. Equivalently, Theorem 7.1 states that, if $v_{\varepsilon}$ is a critical point in $\mathscr{E}_{1}$ obtained by the mountain pass approach in $\Omega$ and for the original energy $E_{\varepsilon}$, then the family $\left(v_{\varepsilon}\right)$ converges (possibly up to a subsequence) as $\varepsilon \rightarrow \infty$, strongly in $H^{1}(\Omega)$, to a maximizer of

$$
M:=\max _{\substack{a \in \Omega \\ \alpha \in \mathbb{S}^{1}}} \int_{\Omega}\left(1-\left|M_{\alpha, a, \Phi}\right|^{2}\right)^{2} .
$$

Before proceeding to the proof, let us note the following. Let $H$ be the map given by Lemma 5.2. Then the restriction $H^{(r)}$ of $H$ to $\overline{\mathbb{D}}_{r}$ is a competitor in (7.2). Thus (with $\beta=\beta_{\varepsilon}$ )

$$
c_{\varepsilon}^{*} \leq \max _{\overline{\mathbb{D}}_{r}} F_{\beta}\left(T\left(N_{0} e^{\imath H^{(r)}}\right)\right)=\max _{a \in \overline{\mathbb{D}}_{r}} F_{\beta}\left(T\left(N_{a}\right)\right) .
$$

\footnotetext{
${ }^{28}$ It will be shown in the proof of Theorem 7.1 that for sufficiently large $\varepsilon$ the value of $r$ can be chosen independent of $\varepsilon$.
} 
Proof. Step 1. We have

$$
c_{\varepsilon}^{*}=\pi+\frac{1}{4 \varepsilon^{2}} M+o\left(\frac{1}{\varepsilon^{2}}\right) \quad \text { as } \varepsilon \rightarrow \infty
$$

Indeed, on the one hand the upper bound $c_{\varepsilon}^{*} \leq \pi+\frac{1}{4 \varepsilon^{2}} M$ is a consequence of (7.7) combined with the fact that

$$
F_{\beta}\left(T\left(N_{a}\right)\right)=\min \left\{F_{\beta}(u) ; \operatorname{tr} u=N_{a}\right\} \leq F_{\beta}\left(M_{a}\right)=\pi+\frac{1}{4 \varepsilon^{2}} \int_{\mathbb{D}} w\left(1-\left|M_{a}\right|^{2}\right)^{2} .
$$

We obtain the upper bound by considering, as a competitor in (7.7), the map $a \mapsto H(a), a \in \overline{\mathbb{D}}_{r}$, (with $H$ as in Lemma 5.2), and by using (7.9).

For the lower bound, fix some $\xi \in \overline{\mathbb{D}}_{r}$ such that $M_{\xi}$ is a maximizer in (7.6). Let $F$ be a competitor in (7.7) such that $J^{*}(F(a))<c_{\varepsilon}^{*}+\frac{1}{\varepsilon^{4}}$ for each $a \in \overline{\mathbb{D}}_{r}$. Clearly, $u \mapsto \widetilde{u}$ is continuous from $H^{1}(\mathbb{D} ; \mathbb{C})$ into itself. By Theorem 4.1 3., for large $\varepsilon$ the map

$$
G: \overline{\mathbb{D}}_{r} \rightarrow \mathbb{D}, G(a)=\text { the zero of the harmonic extension } \underline{F(a)} \text { of } N_{0} e^{\imath F(a)}
$$

is continuous and satisfies $G(a)=a$ if $|a|=r$. By the Brouwer fixed point theorem, we may find some $a=a_{\varepsilon}$ such that $\underline{F}(a)$ vanishes at $z=\xi$. Since $\frac{1}{2} \int_{\mathbb{D}}|\nabla \underline{F}(a)|^{2} \leq c_{\varepsilon}^{*}$, we find, via Corollary 4.6 and Corollary 3.2, that $\underline{F}(a)$ (which depends on $\varepsilon$ ) converges, as $\varepsilon \rightarrow 0$, strongly in $H^{1}(\mathbb{D})$ to $M_{\alpha, \xi}$ for some $\alpha \in \mathbb{S}^{1}$. We find that

$$
\frac{1}{2} \int_{\mathbb{D}}|\nabla \underline{F}(a)|^{2}+\frac{1}{4 \varepsilon^{2}} \int_{\mathbb{D}} w\left(1-|\underline{F}(a)|^{2}\right)^{2} \geq \pi+\frac{1}{4 \varepsilon^{2}} M+o\left(\frac{1}{\varepsilon^{2}}\right) \quad \text { as } \varepsilon \rightarrow \infty .
$$

The lower bound in (7.8) is obtained by combining (7.10) with Lemma 7.3 below. In order to state this lemma, and for further use, let us introduce the following class.

$$
Z_{\varepsilon}=\left\{u \in \mathscr{G} ;-\Delta u=\frac{1}{\varepsilon^{2}} w u\left(1-|u|^{2}\right)\right\} .
$$

7.3 Lemma. Let $u \in Z_{\varepsilon}$. Then

$$
\begin{aligned}
& \|\nabla u-\nabla \widetilde{u}\|_{L^{2}(\mathbb{D})}+\|u-\widetilde{u}\|_{L^{\infty}(\mathbb{D})}=O\left(\frac{1}{\varepsilon^{2}}\right) \quad \text { as } \varepsilon \rightarrow \infty, \\
& \int_{\mathbb{D}}|\nabla u|^{2}=\int_{\mathbb{D}}|\nabla \widetilde{u}|^{2}+O\left(\frac{1}{\varepsilon^{4}}\right) \quad \text { as } \varepsilon \rightarrow \infty,
\end{aligned}
$$

and

$$
\int_{\mathbb{D}} w\left(1-|u|^{2}\right)^{2}=\int_{\mathbb{D}} w\left(1-|\widetilde{u}|^{2}\right)^{2}+O\left(\frac{1}{\varepsilon^{2}}\right) \quad \text { as } \varepsilon \rightarrow \infty .
$$


Proof of Lemma 7.3. Let $v=u-\widetilde{u}$. Let $p>2$. By combining the equation (5.9) satisfied by every $u \in Z_{\varepsilon}$ with the maximum principle Lemma 2.17, with the Sobolev embeddings and with standard elliptic estimates [27, Theorem 9.15, p. 241], we have

$$
\|\nabla v\|_{L^{2}(\mathbb{D})}+\|v\|_{L^{\infty}(\mathbb{D})} \leq C\|\Delta v\|_{L^{p}(\mathbb{D})}=C\|\Delta u\|_{L^{p}(\mathbb{D})} \leq \frac{C^{\prime}}{\varepsilon^{2}}
$$

i.e., (7.12) holds.

We obtain (7.13) by combining (7.12) with the identity $\int_{\mathbb{D}}|\nabla u|^{2}=\int_{\mathbb{D}}|\nabla \widetilde{u}|^{2}+\int_{\mathbb{D}}|\nabla v|^{2}$.

Finally, we note that (by the maximum principle Lemma 2.17) we have $|u| \leq 1,|\widetilde{u}| \leq 1$ and therefore $|v| \leq|u|+|\widetilde{u}| \leq 2$. We find that

$$
\int_{\mathbb{D}} w\left(1-|u|^{2}\right)^{2}-\int_{\mathbb{D}} w\left(1-|\widetilde{u}|^{2}\right)^{2}=-\int_{\mathbb{D}} w\left(2 \widetilde{u} \cdot v+|v|^{4}\right)=O\left(\|v\|_{L^{2}(\mathbb{D})}+\|v\|_{L^{4}(\mathbb{D})}^{2}\right)=O\left(\frac{1}{\varepsilon^{2}}\right) \text { as } \varepsilon \rightarrow \infty
$$

(by (7.12) and $|v| \leq 2$ ), and therefore (7.14) holds.

Proof of Theorem 7.1 continued. To summarize: we have the expansion (7.8) of the energy. Using the identity $|\nabla u|^{2}=2 \mathrm{Jac} u+4\left|\partial_{\bar{z}} u\right|^{2}$ and formula (2.8), we find that

$$
2 \int_{\mathbb{D}}\left|\partial_{\bar{z}} u_{\varepsilon}\right|^{2}+\frac{1}{4 \varepsilon^{2}} \int_{\mathbb{D}} w\left(\left|u_{\varepsilon}\right|^{2}-1\right)^{2}=\frac{1}{4 \varepsilon^{2}} M+o\left(\frac{1}{\varepsilon^{2}}\right) \quad \text { as } \varepsilon \rightarrow \infty .
$$

In order to complete the proof of Theorem 7.1, it suffices to show that

$$
\int_{\mathbb{D}}\left|\partial_{\bar{z}} u_{\varepsilon}\right|^{2}=o\left(\frac{1}{\epsilon^{2}}\right) \quad \text { as } \varepsilon \rightarrow \infty
$$

Indeed, assume for the moment that (7.16) holds. As in the proof of Lemma 6.2, Step 1., we see that any possible weak limit of the $u_{\varepsilon}$ 's is either a constant of modulus 1 , or a Moebius map $M_{\alpha, a}$. By (7.15) and (7.16), the former case cannot hold. By (7.8), (7.15), (7.16) and Corollary 3.2, we find that $u_{\varepsilon} \rightarrow M_{\alpha, a}$ in $H^{1}(\mathbb{D})$, where $M_{\alpha, a}$ is a maximizer in (7.6).

Step 2 in the proof of Theorem 7.1. Proof of (7.16). This proof is similar to the analysis performed in [7, Section 5] in somewhat different context of magnetic Ginzburg-Landau functional in a doubly connected domain.

Introduce $h_{\varepsilon}$ as a solution of

$$
\left\{\begin{array}{ll}
\nabla^{\perp} h_{\varepsilon}=u_{\varepsilon} \wedge \nabla u_{\varepsilon} & \text { in } \mathbb{D} \\
h_{\varepsilon}=0 & \text { on } \mathbb{S}^{1}
\end{array} .\right.
$$

Existence of $h_{\varepsilon}$ follows from the fact that (by (2.23)) we have $\operatorname{div}\left(u_{\varepsilon} \wedge \nabla u_{\varepsilon}\right)=0$ in $\mathbb{D}$ and $u_{\varepsilon} \wedge \frac{\partial u_{\varepsilon}}{\partial v}=0$ on $\mathbb{S}^{1}$. Define

$$
v_{\varepsilon}=\frac{1-\left|u_{\varepsilon}\right|^{2}}{2}+h_{\varepsilon}
$$

The following is straightforward and left to the reader.

7.4 Lemma. We have

$$
\left\{\begin{array}{ll}
\Delta h_{\varepsilon}=2 \mathrm{Jac} u_{\varepsilon} & \text { in } \mathbb{D} \\
h_{\varepsilon}=0 & \text { on } \mathbb{S}^{1}
\end{array},\right.
$$




$$
\begin{cases}\Delta v_{\varepsilon}=\frac{w}{\varepsilon^{2}}\left|u_{\varepsilon}\right|^{2}\left(1-\left|u_{\varepsilon}\right|^{2}\right)-4\left|\partial_{\bar{z}} u_{\varepsilon}\right|^{2} & \text { in } \mathbb{D} \\ v_{\varepsilon}=0 & \text { on } \mathbb{S}^{1}\end{cases}
$$

and

$$
\left|\nabla v_{\varepsilon}\right|^{2}=4\left|u_{\varepsilon}\right|^{2}\left|\partial_{\bar{z}} u_{\varepsilon}\right|^{2}
$$

The key ingredient of the proof of Step 2. is the following

7.5 Lemma. We have

$$
\left\|v_{\varepsilon}\right\|_{L^{\infty}(\mathbb{D})} \rightarrow 0 \quad \text { as } \varepsilon \rightarrow \infty .
$$

Proof of Lemma 7.5. For large $\varepsilon$, let $a=a_{\varepsilon}$ be the unique zero of $\widetilde{u_{\varepsilon}}$ (cf Theorem 4.1 1.). Set $U_{\varepsilon}=$ $u_{\varepsilon} \circ M_{-a}$ and $H_{\varepsilon}=h_{\varepsilon} \circ M_{-a}$. In view of (7.17), we have $v_{\varepsilon} \circ M_{-a}=\frac{1-\left|U_{\varepsilon}\right|^{2}}{2}+H_{\varepsilon}$, and thus (7.21) amounts to

$$
\frac{1-\left|U_{\varepsilon}\right|^{2}}{2}+H_{\varepsilon} \rightarrow 0 \quad \text { uniformly in } \overline{\mathbb{D}} \text { as } \varepsilon \rightarrow \infty \text {. }
$$

As in Step 1., Corollaries 4.6 and 3.2 combined with (7.8) imply that, up to a subsequence,

$$
\widetilde{U_{\varepsilon}} \rightarrow M_{\gamma, 0}=\gamma \text { Id } \quad \text { strongly in } H^{1}(\mathbb{D}) \text { as } \varepsilon \rightarrow \infty \text {. }
$$

By (7.12), we also have

$$
U_{\varepsilon} \rightarrow M_{\gamma, 0}=\gamma \text { Id } \quad \text { strongly in } H^{1}(\mathbb{D}) \text { as } \varepsilon \rightarrow \infty .
$$

Using Lemma 2.19 1. combined with (7.24) and with the fact that (by (7.18)) $H_{\varepsilon}$ satisfies

$$
\left\{\begin{array}{ll}
\Delta H_{\varepsilon}=2 \mathrm{Jac} U_{\varepsilon} & \text { in } \mathbb{D} \\
H_{\varepsilon}=0 & \text { on } \mathbb{S}^{1}
\end{array},\right.
$$

we find that

$$
H_{\varepsilon}(z) \rightarrow \frac{1}{2}\left(|z|^{2}-1\right) \quad \text { uniformly in } \overline{\mathbb{D}} \text { as } \varepsilon \rightarrow \infty \text {. }
$$

On the other hand, by combining Lemma 2.13 with (7.23), we find that

$$
\left|\widetilde{U_{\varepsilon}}(z)\right| \rightarrow|z| \quad \text { uniformly in } \overline{\mathbb{D}} \text { as } \varepsilon \rightarrow \infty \text {. }
$$

The above convergence combined with (7.12) implies that

$$
\left|U_{\varepsilon}(z)\right| \rightarrow|z| \quad \text { uniformly in } \overline{\mathbb{D}} \text { as } \varepsilon \rightarrow \infty \text {. }
$$

Assertion (7.22) (and thus also assertion (7.21)) is obtained by combining (7.26) with (7.25). 
Step 2 continued. If we multiply (7.19) by $v_{\varepsilon}$ and take (7.15), (7.20) and Lemma 7.5 into account, we find that

$$
\int_{\mathbb{D}}\left|u_{\varepsilon}\right|^{2}\left|\partial_{\bar{z}} u_{\varepsilon}\right|^{2}=o\left(\frac{1}{\varepsilon^{2}}\right) \quad \text { as } \varepsilon \rightarrow \infty
$$

In view of (7.26) and of the conformal invariance of the integral in (7.27), estimate (7.27) implies in particular that

$$
\int_{\mathbb{D} \backslash \mathbb{D}_{1 / 2}}\left|\partial_{\bar{z}} U_{\varepsilon}\right|^{2}=o\left(\frac{1}{\varepsilon^{2}}\right) \quad \text { as } \varepsilon \rightarrow \infty .
$$

Therefore, in order to complete the proof of Step 2. it suffices to prove that

$$
\int_{\mathbb{D}_{1 / 2}}\left|\partial_{\bar{z}} U_{\varepsilon}\right|^{2}=o\left(\frac{1}{\varepsilon^{2}}\right) \quad \text { as } \varepsilon \rightarrow \infty
$$

Estimate (7.29) is obtained via the equation satisfied by $\partial_{\bar{z}} U_{\varepsilon}$. In view of (5.9), we have

$$
\Delta\left(\partial_{\bar{z}} U_{\varepsilon}\right)=\frac{1}{\varepsilon^{2}} \partial_{\bar{z}}(\underbrace{\beta \circ M_{-a_{\varepsilon}} \operatorname{Jac} M_{-a_{\varepsilon}} U_{\varepsilon}\left(1-\left|U_{\varepsilon}\right|^{2}\right)}_{\gamma_{\varepsilon}}) \text { in } \mathbb{D} \text {. }
$$

We next invoke the following standard estimate. If $\omega$ is relatively compact in $\Omega$, and if $-\Delta u=f$ in $\Omega$, then

$$
\|\nabla u\|_{L^{2}(\omega)} \leq C\|u\|_{L^{2}(\Omega \backslash \omega)}+C\|f\|_{L^{2}(\Omega) \cdot}{ }^{29}
$$

Noting that $\gamma_{\varepsilon}$ is uniformly bounded on compact subsets of $\mathbb{D}$, we find, via (7.28) and (7.31), that

$$
\left\|\partial_{\bar{z}} U_{\varepsilon}\right\|_{L^{2}\left(\mathbb{D}_{1 / 2}\right)} \leq C\left\|\Delta\left(\partial_{\bar{z}} U_{\varepsilon}\right)\right\|_{L^{2}\left(\mathbb{D}_{3 / 4}\right)}+C\left\|\partial_{\bar{z}} U_{\varepsilon}\right\|_{L^{2}\left(\mathbb{D}_{3 / 4} \backslash \mathbb{D}_{1 / 2}\right)} \leq C \frac{1}{\varepsilon^{2}}+o\left(\frac{1}{\varepsilon}\right) \quad \text { as } \varepsilon \rightarrow \infty .
$$

This implies (7.29). The proof of Theorem 7.1 is complete.

7.6 Remark. The construction of critical points of $E_{\varepsilon}$ in $\mathscr{E}_{d}$ and Theorem 7.1 can be generalized to the case of magnetic Ginzburg-Landau functional, whose minimizers with prescribed degrees were studied in [8] for $\varepsilon \geq \sqrt{2}$. This shows that critical points with prescribed degree one still exist when $\varepsilon<\sqrt{2}$. However, their type changes when passing the critical value $\sqrt{2}$, namely when $\varepsilon>\sqrt{2}$ we have minimizers while for $\varepsilon<\sqrt{2}$ they become minimax critical points.

\footnotetext{
29 This estimate is obtained as follows. We assume for simplicity that $u$ is real-valued. We multiply the equation $-\Delta u=f$ by $\zeta^{2} u$, where $\zeta \in C_{c}^{\infty}(\Omega ; \mathbb{R})$ is fixed such that $\zeta=1$ in $\omega$. After some straightforward calculations, we find that

$$
\int_{\Omega}|\nabla(\zeta u)|^{2}=\int_{\Omega} \zeta^{2} f u+2 \int_{\Omega} \zeta u \nabla u \cdot \nabla \zeta
$$
}

and this leads to

$$
\int_{\Omega}|\nabla(\zeta u)|^{2} \leq C_{1}\|u\|_{L^{2}(\Omega \backslash \omega)}^{2}+\varepsilon\|\zeta u\|_{L^{2}(\Omega)}^{2}+C(\varepsilon)\|f\|_{L^{2}(\Omega)}^{2} .
$$

We obtain (7.31) by combining (7.32) with Poincaré's inequality $\|\zeta u\|_{L^{2}(\Omega)} \leq C\|\nabla(\zeta u)\|_{L^{2}(\Omega)}$. 


\section{Bubbling analysis for small $\varepsilon$}

When $\varepsilon$ is small instead of large, our proof of Theorem 1.1 breaks down, although we expect its conclusion to remain true. In fact the very definition of $J^{*}$ is valid only for large $\varepsilon$ since it requires the uniqueness of solutions of (5.9).

Nevertheless we present in this section an alternative minimax setting which is valid for any $\varepsilon>0$, and for which the proof of Theorem 1.1 can be carried out up to and including the analysis of Palais-Smale sequences, i.e., the analog of Corollary 4.6. We believe this result is interesting in its own right.

Let $X^{\sharp}:=H_{0}^{1}(\mathbb{D} ; \mathbb{C}) \times H^{1 / 2}\left(\mathbb{S}^{1} ; \mathbb{R}\right)$ and

$$
U: X^{\sharp} \rightarrow H^{1}(\mathbb{D} ; \mathbb{C}), \quad X^{\sharp} \ni(v, \psi) \mapsto U(v, \psi):=v+u\left(N_{0} e^{\imath \psi}\right) .
$$

Recall that $N_{0}$ is the identity of $\mathbb{S}^{1}$ and that $u(w)$ is the harmonic extension of $w$. Set $J^{\sharp}(v, \psi):=$ $F_{\beta}(U(v, \psi))$.

As in Section 5 , we let $K=\overline{\mathbb{D}}_{r}, K_{0}=C_{r}$ and we write $N_{a}=N_{0} e^{\imath \psi_{a}}$. Define

$$
\chi^{\sharp}: C_{r} \rightarrow X^{\sharp}, \quad C_{r} \ni a \mapsto \chi^{\sharp}(a)=\left(0, \psi_{a}\right) .
$$

We have $U \circ \chi^{\sharp}(a)=M_{a}$.

We also define

$$
c^{\sharp}:=\inf \left\{\max _{K} J^{\sharp} \circ F ; F \in C\left(K ; X^{\sharp}\right), F=\chi^{\sharp} \text { on } K_{0}\right\}, \quad c_{1}^{\sharp}:=\max _{K_{0}} J^{\sharp} \circ \chi^{\sharp} .
$$

8.1 Remark. Assume, just in this remark, that (5.8) holds. Then we clearly have $c^{\sharp}=c^{*}$ and $c_{1}^{\sharp}=c_{1}^{*}$, with $c^{*}$ and $c_{1}^{*}$ as in (5.10).

By repeating the proof of Lemma 5.3, we find the following

8.2 Lemma. Assume (4.14). Then, for $r$ sufficiently close to 1 , we have $c^{\sharp}>c_{1}^{\sharp}$.

We next establish the analogs of Lemma 5.8 and of Corollary 5.12 when $J^{*}$ is replaced by $J^{\sharp}$; this will require more involved arguments. We start with some straightforward consequences of Lemma 5.4 .

8.3 Lemma. Let $1 \leq p<\infty$. Let $u_{0} \in H^{1 / 2} \cap L^{\infty}\left(\mathbb{S}^{1} ; \mathbb{C}\right)$. Then the map

$$
\mathscr{F}_{1}: H^{1 / 2}\left(\mathbb{S}^{1} ; \mathbb{R}\right) \rightarrow L^{p}\left(\mathbb{S}^{1} ; \mathbb{C}\right), \quad H^{1 / 2}\left(\mathbb{S}^{1} ; \mathbb{R}\right) \ni \psi \stackrel{\mathscr{F}_{1}}{\longrightarrow} u_{0} e^{\imath \psi}
$$

is $C^{1}$, and $\mathscr{F}_{1}^{\prime}(\psi)(\eta)=\imath u_{0} e^{\imath \psi} \eta$.

Proof. The point to be checked is that $\mathscr{T}(\psi)(\eta):=\imath u_{0} e^{\imath \psi} \eta$ defines a map $\mathscr{T} \in C\left(H^{1 / 2} ; B\left(H^{1 / 2} ; L^{p}\right)\right)$. This follows from

$$
\left\|\mathscr{T}\left(\psi_{1}\right)-\mathscr{T}\left(\psi_{2}\right)\right\| \leq \sup _{\|\eta\|_{H^{1 / 2}} \leq 1}\left\|\left(\psi_{1}-\psi_{2}\right) \eta\right\|_{L^{p}} \leq \sup _{\|\eta\|_{H^{1 / 2}} \leq 1}\left\|\psi_{1}-\psi_{2}\right\|_{L^{2 p}}\|\eta\|_{L^{2 p}} \leq C\left\|\psi_{1}-\psi_{2}\right\|_{H^{1 / 2}},
$$

by the Sobolev embedding $H^{1 / 2}\left(\mathbb{S}^{1}\right) \hookrightarrow L^{2 p}\left(\mathbb{S}^{1}\right)$. 
8.4 Lemma. Let $1 \leq p<\infty$. Let $u_{0} \in H^{1 / 2} \cap L^{\infty}\left(\mathbb{S}^{1} ; \mathbb{C}\right)$. Then the map

$$
\mathscr{F}_{2}: H_{0}^{1}(\mathbb{D} ; \mathbb{C}) \times H^{1 / 2}\left(\mathbb{S}^{1} ; \mathbb{R}\right) \rightarrow L^{p}(\mathbb{D}), \quad H_{0}^{1}(\mathbb{D} ; \mathbb{C}) \times H^{1 / 2}\left(\mathbb{S}^{1} ; \mathbb{R}\right) \ni(v, \psi) \stackrel{\mathscr{F}_{2}}{\longrightarrow} v+u\left(u_{0} e^{\imath \psi}\right),
$$

is $C^{1}$, and $\mathscr{F}_{2}^{\prime}(v, \psi)(w, \eta)=w+u\left(\imath u_{0} e^{\imath \psi} \eta\right)$.

Proof. It suffices to combine the previous lemma with the embedding $H^{1}(\mathbb{D}) \hookrightarrow L^{p}(\mathbb{D})$ and with the continuity of the map $L^{p}\left(\mathbb{S}^{1}\right) \ni f \mapsto u(f) \in L^{p}(\mathbb{D})$.

By combining the above result with Lemma 5.5, we obtain

8.5 Lemma. With $u_{0}$ as above and $\beta \in L^{\infty}(\mathbb{D})$, the map

$$
\mathscr{F}_{3}: H_{0}^{1}(\mathbb{D} ; \mathbb{C}) \times H^{1 / 2}\left(\mathbb{S}^{1} ; \mathbb{R}\right) \rightarrow \mathbb{R}, \quad H_{0}^{1}(\mathbb{D} ; \mathbb{C}) \times H^{1 / 2}\left(\mathbb{S}^{1} ; \mathbb{R}\right) \ni(v, \psi) \stackrel{\mathscr{F}_{3}}{\longrightarrow} \frac{1}{4} \int_{\mathbb{D}} \beta\left(1-\left|v+u\left(u_{0} e^{\imath \psi}\right)\right|^{2}\right)^{2},
$$

is $C^{1}$, and, if we set $u:=v+u\left(u_{0} e^{\imath \psi}\right)$, then

$$
\mathscr{F}_{3}{ }^{\prime}(v, \psi)(w, \eta)=-\int_{\mathbb{D}} \beta u \cdot\left(w+u\left(\imath u_{0} e^{\imath \psi} \eta\right)\right)\left(1-|u|^{2}\right) .
$$

8.6 Lemma. The map

$$
\mathscr{F}_{4}: H^{1 / 2}\left(\mathbb{S}^{1} ; \mathbb{R}\right) \rightarrow \mathbb{R}, \quad H^{1 / 2}\left(\mathbb{S}^{1} ; \mathbb{R}\right) \ni \psi \stackrel{\mathscr{F}_{4}}{\longrightarrow} \frac{1}{2} \int_{\mathbb{D}}\left|\nabla u\left(u_{0} e^{\imath \psi}\right)\right|^{2},
$$

is $C^{1}$, and, if $u=u\left(u_{0} e^{\imath \psi}\right)$, then

$$
\mathscr{F}_{4}^{\prime}(\psi)(\eta)=\int_{\mathbb{S}^{1}}\left(u \wedge \frac{\partial u}{\partial v}\right) \eta=\int_{\mathbb{D}}(u \wedge \nabla u) \cdot \nabla \zeta, \forall \psi, \eta \in H^{1 / 2}\left(\mathbb{S}^{1} ; \mathbb{R}\right), \forall \zeta \in H^{1}(\mathbb{D} ; \mathbb{R}) \text { such that } \operatorname{tr} \zeta=\eta .
$$

Proof. The equality of the two integrals in (8.2) is justified as in the proof of (5.14).

In order to prove that $\mathscr{F}_{4}{ }^{\prime}(\psi)(\eta)=\mathscr{T}(\psi)(\eta)$, where $\mathscr{T}(\psi)(\eta):=\int_{\mathbb{D}}(u \wedge \nabla u) \cdot \nabla u(\eta)$, we rely on Lemma 5.4. The map $\psi \mapsto u \wedge \nabla u \in L^{2}(\mathbb{D})$ is continuous, ${ }^{30}$ and thus $\mathscr{T} \in C\left(H^{1 / 2} ; H^{-1 / 2}\right)$. On the other hand, it is clear that $\mathscr{F}_{4}$ is continuous. Therefore, it remains to prove that (8.2) holds when $\psi$ and $\eta$ are smooth. By replacing $u_{0}$ with $u_{0} e^{\imath \psi}$, we may assume that $\psi=0$. We have

$$
u_{0} e^{\imath t \eta}=u_{0}(1+\imath t \eta)+R(t), \quad \text { with }\|R(t)\|_{H^{1 / 2}} \leq C t^{2} \text { as } t \rightarrow 0,
$$

and thus

$$
\mathscr{F}_{4}(t \eta)=\frac{1}{2} \int_{\mathbb{D}}|\nabla u|^{2}+t \int_{\mathbb{D}} \nabla u \cdot \nabla u\left(\imath u_{0} \eta\right)+S(t), \quad \text { with }\|S(t)\|_{H^{1 / 2}} \leq C t^{2} \text { as } t \rightarrow 0 .
$$

Using (8.3) and (5.16) with $\beta=0$, we find that

$$
\frac{\partial \mathscr{F}_{4}}{\partial \eta}(0)=\int_{\mathbb{D}} \nabla u \cdot \nabla\left(\iota u_{0} \eta\right)=\int_{\mathbb{S}^{1}}\left(u \wedge \frac{\partial u}{\partial v}\right) \eta=\mathscr{T}(0)(\eta) .
$$

\footnotetext{
${ }^{30}$ This argument was already used in the proof of Lemma 5.7.
} 
An immediate consequence of Lemma 8.6 is the analog of Lemma 5.8.

8.7 Lemma. The map $J^{\sharp}$ is $C^{1}$. In addition, with $u=v+u\left(N_{0} e^{\imath \psi}\right)$ and with $U=u\left(N_{0} e^{\imath \psi}\right)$, we have

$$
J^{\sharp \prime}(v, \psi)(w, \eta)=\int_{\mathbb{D}} \nabla v \cdot \nabla w+\int_{\mathbb{D}}(U \wedge \nabla U) \cdot \nabla \zeta-\int_{\mathbb{D}} \beta u \cdot\left(w+u\left(\imath N_{0} e^{\imath \psi} \eta\right)\right)\left(1-|u|^{2}\right)
$$

for every $\zeta \in H^{1}(\mathbb{D})$ such that $\operatorname{tr} \zeta=\eta$.

Proof. It suffices to note that $J^{\sharp}(v, \psi)=\mathscr{F}_{4}(\psi)+\frac{1}{2} \int_{\mathbb{D}}|\nabla v|^{2}$, with $\mathscr{F}_{4}$ as in Lemma 8.6. ${ }^{31}$

We next turn to the properties of the Palais-Smale sequences associated to $J^{\sharp}$. Note that an application of Theorem 5.1 in conjunction with Lemma 8.2 leads to a sequence $\left(v_{n}, \psi_{n}\right)$ such that, with $u_{n}^{*}:=v_{n}+u\left(N_{0} e^{\imath \psi_{n}}\right) \in \mathscr{G}$, we have

$$
J^{\sharp \prime}\left(v_{n}, \psi_{n}\right) \rightarrow 0, J^{\sharp}\left(v_{n}, \psi_{n}\right) \rightarrow c^{\sharp}, u_{n}^{*} \rightarrow u \text { in } H^{1} \text { as } n \rightarrow \infty .
$$

In the asymptotic analysis of the Palais-Smale sequences, the smoothness of $u_{n}^{*}$ is not sufficient. In the remaining part of this section, we find a better Palais-Smale sequence and establish its main properties. More specifically, we let $u_{n}:=v+u\left(N_{0} e^{\imath \psi_{n}}\right)$, where $v \in H_{0}^{1}(\mathbb{D})$ is such that $v_{n} \rightarrow v$ as $n \rightarrow \infty$. In the original variables of $\boldsymbol{J}^{\sharp}$, this amounts to replacing $v_{n}$ by $v$. The next result is the analog of Corollary 5.12.

8.8 Lemma. Assume that $\left(v_{n}, \psi_{n}\right)$ satisfy (8.5), with $u_{n}^{*}:=v_{n}+u\left(N_{0} e^{\imath \psi_{n}}\right)$, and that $v_{n} \rightarrow v$ as $n \rightarrow \infty$. Then, letting

$$
u_{n}:=v+u\left(N_{0} e^{\imath \psi_{n}}\right)
$$

the sequence $\left(u_{n}\right)$ has the following properties.

1. $u$ is a solution of (2.23), and we have $-\Delta v=\beta u\left(1-|u|^{2}\right)$.

2. $\left|u_{n}\right| \leq C$.

3. $-\Delta u_{n}-\beta u_{n}\left(1-\left|u_{n}\right|^{2}\right) \rightarrow 0$ as $n \rightarrow \infty$ in every $L^{p}, p<\infty$.

4. $u_{n}-u_{n}^{*} \rightarrow 0$ as $n \rightarrow \infty$ in $H^{1}(\mathbb{D})$. In particular, $u_{n} \rightarrow u$ and $F_{\beta}\left(u_{n}\right) \rightarrow c^{\sharp}$ as $n \rightarrow \infty$.

5. $\left(u_{n}\right)$ is a Palais-Smale sequence.

6. There exists a sequence $c_{n} \rightarrow 0$ such that

$$
\left|\int_{\mathbb{D}}\left(u_{n} \wedge \nabla u_{n}\right) \cdot \nabla \zeta\right| \leq c_{n}\|\nabla \zeta\|_{L^{2}}, \quad \forall \zeta \in H^{1}(\mathbb{D} ; \mathbb{R}) .
$$

\footnotetext{
${ }^{31}$ With $u_{0}=N_{0}$.
} 
Proof. All items except item 6. are straightforward. Indeed, let $U_{n}:=u\left(N_{0} e^{\imath \psi_{n}}\right)$. Since $u_{n}^{*}=v_{n}+U_{n} \rightarrow$ $u$, we find that $U_{n} \rightarrow U$ as $n \rightarrow \infty$ for some harmonic $U \in \mathscr{G}$, and thus and $v_{n} \rightarrow v:=u-U$ as $n \rightarrow \infty$. Using the fact that $\left(u_{n}^{*}\right)$ is a Palais-Smale sequence, we find, by passing to the limits in (8.4), ${ }^{32}$ that $-\Delta v=\beta u\left(1-|u|^{2}\right)$, whence the last assertion in item 1. Item 2. follows by combining the inequality $\left|U_{n}\right| \leq 1$ with the fact that $v$ is smooth. ${ }^{33}$ Item 3. follows from the fact that $u_{n} \rightarrow u$ as $n \rightarrow \infty$ in $L^{p}$ for every $p<\infty$. This item implies that $u_{n} \rightarrow u$ in $W^{2, p}$ as $n \rightarrow \infty, \forall p<\infty$, and thus item 4 . Item 5 . is an easy consequence of the fact that $v_{n} \rightarrow v$ in $H^{1}$ as $n \rightarrow \infty$ (which in turn follows from item 4.) combined with the fact that $u_{n}^{*}-u_{n} \rightarrow 0$ as $n \rightarrow \infty$ in every $L^{p}$.

We now turn to property 6 ., which is at the heart of the lemma. Since $\Delta U_{n}=0$ in $\mathbb{D}$, we have

$$
\int_{\mathbb{D}}\left(U_{n} \wedge \nabla U_{n}\right) \cdot \nabla \zeta=\int_{\mathbb{D}} \operatorname{div}\left(\left(U_{n} \wedge \nabla U_{n}\right) \zeta\right)=\int_{\mathbb{S}^{1}} \frac{\partial U_{n}}{\partial v} \cdot\left({ }_{\imath} U_{n} \eta\right)=\int_{\mathbb{D}} \nabla U_{n} \cdot \nabla u\left(\imath N_{0} e^{\imath \psi_{n}} \eta\right) .
$$

We plug this into (8.4) and integrate by parts to find, with $\zeta \in H^{1}(\mathbb{D})$ such that $\operatorname{tr} \zeta=\eta$, that

$$
\begin{aligned}
J^{\sharp \prime}\left(v, \psi_{n}\right)(0, \eta) & =\int_{\mathbb{D}} \nabla U_{n} \cdot \nabla u\left(\imath N_{0} e^{\imath \psi_{n}} \eta\right)-\int_{\mathbb{D}} \beta u_{n} \cdot u\left(\imath N_{0} e^{\imath \psi_{n}} \eta\right)\left(1-\left|u_{n}\right|^{2}\right) \\
& =\int_{\mathbb{D}} \nabla u_{n} \cdot \nabla u\left(\imath N_{0} e^{\imath \psi_{n}} \eta\right)-\int_{\mathbb{D}} \beta u_{n} \cdot u\left(\imath N_{0} e^{\imath \psi_{n}} \eta\right)\left(1-\left|u_{n}\right|^{2}\right) \\
& =\int_{\mathbb{S}^{1}} u_{n} \wedge \frac{\partial u_{n}}{\partial v} \eta-\int_{\mathbb{D}}\left(\Delta u_{n}+\beta u_{n}\left(1-\left|u_{n}\right|^{2}\right)\right) \cdot u\left(\imath N_{0} e^{\imath \psi_{n}} \eta\right) \\
& =\int_{\mathbb{D}}\left(u_{n} \wedge \nabla u_{n}\right) \cdot \nabla \zeta+\int_{\mathbb{D}} u_{n} \wedge \Delta u_{n} \zeta-\int_{\mathbb{D}}\left(\Delta u_{n}+\beta u_{n}\left(1-\left|u_{n}\right|^{2}\right)\right) \cdot u\left(\imath N_{0} e^{\imath \psi_{n}} \eta\right) .
\end{aligned}
$$

The above calculation is valid for smooth $\eta$ and $\zeta$. By density, it still holds for every $\eta \in H^{1 / 2}\left(\mathbb{S}^{1} ; \mathbb{R}\right)$ and every $\eta \in H^{1}(\mathbb{D} ; \mathbb{R})$ such that $\operatorname{tr} \zeta=\eta$. In particular, we have

$$
\left|\int_{\mathbb{D}}\left(u_{n} \wedge \nabla u_{n}\right) \cdot \nabla \zeta\right| \leq\left|J^{\sharp \prime}\left(v, \psi_{n}\right)(0, \eta)\right|+\int_{\mathbb{D}}\left|u_{n} \wedge \Delta u_{n}\right||\zeta|+\int_{\mathbb{D}}\left|\Delta u_{n}+\beta u_{n}\left(1-\left|u_{n}\right|^{2}\right)\right|\left|u\left(\imath N_{0} e^{\imath \psi_{n}} \eta\right)\right| .
$$

We take, in the above inequality, $\zeta$ with zero mean and such that $\eta=\operatorname{tr} \zeta$. With this choice, if we invoke Poincaré's inequality, combined with the bounds

$$
|\eta|_{H^{1 / 2}} \leq C\|\nabla \zeta\|_{L^{2}} \text { and }\left\|u\left({ }_{l} N_{0} e^{\imath \psi_{n}} \eta\right)\right\|_{L^{p}} \leq C|\eta|_{H^{1 / 2}}, \forall p<\infty,
$$

and with items 3 . and 5., then we obtain item 6 .

Finally, the first assertion in item 1 . follows by combining 6 . with the second part of item 1 .

8.9 Definition. In the following we will denote by the term bubble either a Moebius transform or the conjugate of a Moebius transform. We will denote such a bubble by $\mathscr{B}_{a}$, with $a \in \mathbb{D}$. Thus either $\mathscr{B}_{a}=M_{a}$ or $\mathscr{B}_{a}=\overline{M_{a}}$.

A multi-bubble is either a (non trivial) Blaschke product, or the conjugate of such a product.

The key result in the analysis of the Palais-Smale sequences associated to $J^{\sharp}$ is the following.

\footnotetext{
${ }^{32}(8.4)$ is applied to the couple $\left(v_{n}, \psi_{n}\right)$, with $\eta=0$ and for fixed $w$.

${ }^{33}$ Here, we use Lemmas 2.17 and 2.15 .
} 
8.10 Lemma. Let $\left(v_{n}\right) \subset \mathscr{G}$ be a sequence of harmonic functions with the following properties:

$$
\begin{aligned}
& v_{n} \rightarrow 1 \quad \text { in } H^{1}(\mathbb{D}) \text { as } n \rightarrow \infty \\
& \frac{1}{2} \int_{\mathbb{D}}\left|\nabla v_{n}\right|^{2} \rightarrow K \pi \text { as } n \rightarrow \infty \\
& \left|\int_{\mathbb{D}}\left(v_{n} \wedge \nabla v_{n}\right) \cdot \nabla \zeta\right| \leq c_{n}\|\nabla \zeta\|_{L^{2}}, \quad \forall \zeta \in H^{1}(\mathbb{D} ; \mathbb{R}), \text { with } c_{n} \rightarrow 0 \text { as } n \rightarrow \infty .
\end{aligned}
$$

Then $K$ is an integer and, up to a subsequence, there exist points $a_{1}(n), \ldots, a_{K}(n)$, corresponding bub-

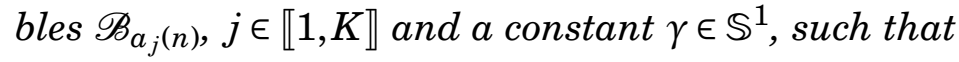

$$
\begin{aligned}
& \left|a_{j}(n)\right| \rightarrow 1 \text { as } n \rightarrow \infty, \forall j \in \llbracket 1, K \rrbracket ; \\
& v_{n}-\gamma \prod_{j=1}^{K} \mathscr{B}_{a_{j}(n)} \rightarrow 0 \text { strongly in } H^{1}(\mathbb{D}) \text { as } n \rightarrow \infty .
\end{aligned}
$$

Proof. The proof is by induction on the integer part $[K]$ of $K$.

Step 1. Case where $K<1$.

In this case, we will prove that $v_{n} \rightarrow 1$ strongly in $H^{1}(\mathbb{D})$ as $n \rightarrow \infty$. By Lemma 4.24 ., if $K<1$ then $\left|v_{n}\right| \geq a>0$ for large $n$ and some constant $a$. Thus we may write, globally in $\mathbb{D}, v_{n}=\rho_{n} e^{l \varphi_{n}}$ (Lemma 2.7 3.). If we take $\zeta=\varphi_{n}$ in (8.9), then using (2.11) we find that $\nabla \varphi_{n} \rightarrow 0$ in $L^{2}$ as $n \rightarrow \infty$. Since $v_{n}$ is harmonic, we have $\left\{\begin{array}{ll}\Delta \rho_{n}=\rho_{n}\left|\nabla \varphi_{n}\right|^{2} & \text { in } \mathbb{D} \\ \rho_{n}=1 & \text { on } \mathbb{S}^{1}\end{array}\right.$, and therefore $\rho_{n} \rightarrow 1$ strongly in $H^{1}(\mathbb{D})$ as $n \rightarrow \infty .{ }^{34}$ Using (2.11) and the facts that $\nabla \varphi_{n} \rightarrow 0$ and $\nabla \rho_{n} \rightarrow 0$ in $L^{2}$, we obtain that $v_{n} \rightarrow 1$ as $n \rightarrow \infty$ strongly in $H^{1}$, i.e., that (8.11) holds with $K=0$.

Step 2. Induction step.

Assume that $[K] \geq 1$. Then the $H^{1}$ convergence of $\left(v_{n}\right)$ (or any subsequence of $\left(v_{n}\right)$ ) to 1 is weak, but not strong. By the argument developed in Step 1., there exists no $a>0$ such that $\left|v_{n}\right| \geq a$ holds along a subsequence. Thus we may pick $z_{n} \in \mathbb{D}$ such that $v_{n}\left(z_{n}\right) \rightarrow 0$. In view of (8.7), we have $\left|z_{n}\right| \rightarrow 1$ as $n \rightarrow \infty$.

Let $w_{n}=v_{n} \circ M_{-z_{n}}$. The new sequence $\left(w_{n}\right)$ satisfies (8.8), (8.9) and $w_{n}(0) \rightarrow 0$ as $n \rightarrow \infty$.

Let $w$ be the weak limit of $\left(w_{n}\right)$ (possibly along a subsequence). Then $w$ is harmonic, $w(0)=0$ and, by (8.9), $w$ satisfies (2.23) with $\beta=0$. In view of Lemma 3.5, there exist $a_{1}, \ldots, a_{L}$ in $\mathbb{D}$ and a constant $\gamma \in \mathbb{S}^{1}$ such that

$$
w=\gamma M_{a_{1}} \ldots M_{a_{L}}, \quad \text { or } \quad \bar{w}=\gamma M_{a_{1}} \ldots M_{a_{L}} .
$$

In particular, $\frac{1}{2} \int_{\mathbb{D}}|\nabla w|^{2}=L \pi$, with $L$ a positive integer. Let $f:=\operatorname{tr} w$ and $g_{n}:=\operatorname{tr}\left(w_{n} \bar{w}\right)$. Then $g_{n} \rightarrow 1$ in $H^{1 / 2}\left(\mathbb{S}^{1} ; \mathbb{S}^{1}\right)$ as $n \rightarrow \infty$. We also note the following. On the one hand, we have $w_{n}=u\left(f g_{n}\right)$. On the other hand, if we set $h_{n}:=u\left(g_{n}\right)$, then $h_{n} \rightarrow 1$ in $H^{1}(\mathbb{D})$ as $n \rightarrow \infty$ (and also $h_{n} \rightarrow 1$ in $C_{l o c}^{1}(\mathbb{D})$ as $n \rightarrow \infty$.) By Lemmas 2.22 and 2.24, we have $w_{n}-w h_{n} \rightarrow 0$ in $H^{1}(\mathbb{D})$ and $\frac{1}{2} \int_{\mathbb{D}}\left|\nabla h_{n}\right|^{2} \rightarrow(K-L) \pi$ as $n \rightarrow$ $\infty$. We now turn back to the original sequence $\left(v_{n}\right)$. Set $t_{n}:=\overline{\gamma_{n}} w \circ M_{z_{n}}, y_{n}:=h_{n} \circ M_{z_{n}}$, where $\gamma_{n}=w \circ M_{z_{n}}(0) /\left|w \circ M_{z_{n}}(0)\right|$ (note that $\left|w \circ M_{z_{n}}(0)\right|=\left|w\left(-z_{n}\right)\right| \rightarrow 1$ as $n \rightarrow \infty$ ). Then we have:

\footnotetext{
${ }^{34}$ This is obtained by multiplying by $\rho_{n}-1$ the equation of $\rho_{n}$.
} 
1. $v_{n}-\gamma_{n} t_{n} y_{n} \rightarrow 0$ in $H^{1}(\mathbb{D})$ as $n \rightarrow \infty$.

2. Letting $a_{j}(n):=M_{-z_{n}}\left(a_{j}\right)$, we have $\left|a_{j}(n)\right| \rightarrow 1$ as $n \rightarrow \infty$, and either $t_{n}=\overline{\gamma_{n}} \gamma \prod_{j} M_{a_{j}(n)}$, or $\bar{t}_{n}=$ $\gamma_{n} \gamma \prod_{j} M_{a_{j}(n)}$.

3. $\frac{1}{2} \int_{\mathbb{D}}\left|\nabla y_{n}\right|^{2} \rightarrow(K-L) \pi$ as $n \rightarrow \infty$.

4. $y_{n} \rightarrow 1$ in $H^{1}(\mathbb{D})$ as $n \rightarrow \infty$ (since $v_{n} \rightarrow 1$ and $\overline{\gamma_{n}} w \circ M_{z_{n}} \rightarrow 1$ as $n \rightarrow \infty$ ).

In order to complete the proof of Lemma 8.10, we apply the induction hypothesis to the sequence $\left(y_{n}\right)$. To this end we need to establish the validity of (8.9) for the sequence $\left(y_{n}\right)$. This is done as follows. By conformal invariance of (8.9) and the fact that (8.9) holds for $\left(v_{n}\right)$, we find that (8.9) holds for $\left(w_{n}\right)$. On the other hand, (8.12) implies that $\int_{\mathbb{D}}(w \wedge \nabla w) \cdot \nabla \zeta=0$. We next recall that $w_{n}=w h_{n}+r_{n}$, where $r_{n} \rightarrow 0$ in $H^{1}(\mathbb{D})$ as $n \rightarrow \infty$. Since $\Delta r_{n}=-2 \nabla w \cdot \nabla h_{n}$ is bounded in $L^{2}(\Omega)$, the sequence $\left(r_{n}\right)$ is bounded in $W^{1, p}(\mathbb{D}), \forall p \in[1, \infty)$, and thus $r_{n} \rightarrow 0$ in $L^{\infty}(\mathbb{D})$ as $n \rightarrow \infty$. Therefore, we have

$$
w_{n} \wedge \nabla w_{n}=\left|h_{n}\right|^{2}(w \wedge \nabla w)+|w|^{2}\left(h_{n} \wedge \nabla h_{n}\right)+F_{n},
$$

with $F_{n} \in L^{2}\left(\mathbb{D} ; \mathbb{R}^{2}\right)$ satisfying $\left\|F_{n}\right\|_{L^{2}} \rightarrow 0$ as $n \rightarrow \infty$. From this we find that

$$
\begin{aligned}
\int_{\mathbb{D}}\left(h_{n} \wedge \nabla h_{n}\right) \cdot \nabla \zeta= & \int_{\mathbb{D}}\left(w_{n} \wedge \nabla w_{n}\right) \cdot \nabla \zeta+\int_{\mathbb{D}}\left(1-|w|^{2}\right)\left(h_{n} \wedge \nabla h_{n}\right) \cdot \nabla \zeta \\
& +\int_{\mathbb{D}}\left(1-\left|h_{n}\right|^{2}\right)(w \wedge \nabla w) \cdot \nabla \zeta-\int_{\mathbb{D}}(w \wedge \nabla w) \cdot \nabla \zeta-\int_{\mathbb{D}} F_{n} \cdot \nabla \zeta \\
= & \int_{\mathbb{D}}\left(w_{n} \wedge \nabla w_{n}\right) \cdot \nabla \zeta+\int_{\mathbb{D}}\left(1-|w|^{2}\right)\left(h_{n} \wedge \nabla h_{n}\right) \cdot \nabla \zeta \\
& +\int_{\mathbb{D}}\left(1-\left|h_{n}\right|^{2}\right)(w \wedge \nabla w) \cdot \nabla \zeta-\int_{\mathbb{D}} F_{n} \cdot \nabla \zeta .
\end{aligned}
$$

The fact that $h_{n} \rightarrow 1$ in $C_{l o c}^{1}(\mathbb{D})$ and in $L^{4}(\mathbb{D})$ as $n \rightarrow \infty$ and the fact that $|w(z)| \rightarrow 1$ as $|z| \rightarrow 1$ easily imply that

$$
\left|\int_{\mathbb{D}}\left(1-|w|^{2}\right)\left(h_{n} \wedge \nabla h_{n}\right) \cdot \nabla \zeta\right|+\left|\int_{\mathbb{D}}\left(1-\left|h_{n}\right|^{2}\right)(w \wedge \nabla w) \cdot \nabla \zeta\right| \leq c_{n}\|\nabla \zeta\|_{L^{2}}, \quad \text { with } c_{n} \rightarrow 0 \text { as } n \rightarrow \infty .
$$

It follows from (8.13) and (8.14) that $\left(h_{n}\right)$ satisfies (8.9). By conformal invariance of (8.9), the same holds for $\left(y_{n}\right)$.

8.11 Remark. Lemma 8.10 is about bubbling of harmonic functions in the unit disc $\mathbb{D}$, and clearly this analysis extends to the case of simply connected domains. It is still possible to study the case of multiply connected domains. Such analysis, which is not relevant for the subsequent results in this section, is postponed to Section 9 .

8.12 Lemma. Let $\left(v_{n}, \psi_{n}\right)$ be a Palais-Smale sequence associated to $J^{\sharp}$, and set $u_{n}^{*}:=v_{n}+u\left(N_{0} e^{\imath \psi_{n}}\right)$. Assume that $u_{n}^{*} \rightarrow u$ in $H^{1}(\mathbb{D})$ as $n \rightarrow \infty$. Set $g_{n}=\operatorname{tr}\left(u_{n}^{*} \bar{u}\right)$ and $w_{n}=u\left(g_{n}\right)$. Then

1. $u_{n}^{*}-u w_{n} \rightarrow 0$ strongly in $H^{1}(\mathbb{D})$ as $n \rightarrow \infty$.

2. $F_{\beta}\left(u_{n}^{*}\right)=F_{\beta}(u)+\frac{1}{2} \int_{\mathbb{D}}\left|\nabla w_{n}\right|^{2}+c_{n}$, with $c_{n} \rightarrow 0$ as $n \rightarrow \infty$.

3. The sequence $\left(w_{n}\right)$ satisfies the assumptions of Lemma 8.10. 
Proof. Let $u_{n}=v+u\left(N_{0} e^{\imath \psi_{n}}\right)$, where $v=\lim _{n} v_{n}$ as in Lemma 8.8. By Lemma 8.8, item 1. amounts to proving that $z_{n}:=u w_{n}-u_{n}$ converges strongly to 0 in $H^{1}(\mathbb{D})$ as $n \rightarrow \infty$. By combining the fact that $u$ solves (2.21) with Lemma 8.8 (which implies that $-\Delta u_{n}=-\Delta v=\beta u\left(1-|u|^{2}\right)$ ), and with the fact that $w_{n}$ is harmonic, we find that the map $z_{n}$ solves

$$
\left\{\begin{array}{ll}
-\Delta z_{n}=\beta u\left(w_{n}-1\right)\left(1-|u|^{2}\right)-2 \nabla u \cdot \nabla w_{n} & \text { in } \mathbb{D} \\
z_{n}=0 & \text { on } \mathbb{S}^{1}
\end{array} .\right.
$$

Then, by Remark 2.23, we obtain that $z_{n} \rightarrow 0$ in $H^{1}(\mathbb{D})$.

We turn to item 2. By combining item 1. with the fact that $w_{n} \rightarrow 1$ weakly in $H^{1}$ and $w_{n} \rightarrow 1$ strongly in $L^{p}$ as $n \rightarrow \infty, \forall p \in[1, \infty)(p \geq 1)$, we find that item 2 . amounts to proving that, as $n \rightarrow \infty$, we have

$$
\int_{\mathbb{D}}\left|\nabla\left(u w_{n}\right)\right|^{2}=\int_{\mathbb{D}}|\nabla u|^{2}+\int_{\mathbb{D}}\left|\nabla w_{n}\right|^{2}+o(1) .
$$

The starting point in the proof of (8.15) is the identity

$$
\left|\nabla\left(u w_{n}\right)\right|^{2}=|u|^{2}\left|\nabla w_{n}\right|^{2}+|\nabla u|^{2}\left|w_{n}\right|^{2}+2\left(u \nabla w_{n}\right) \cdot\left(w_{n} \nabla u\right) .
$$

Since $w_{n}$ is harmonic and $w_{n}-1$ in $H^{1}$ as $n \rightarrow \infty$, we have $w_{n} \rightarrow 1$ in $C_{l o c}^{1}$ as $n \rightarrow \infty$. On the other hand, we have $|u(x)| \rightarrow 1$ uniformly as $x \rightarrow \partial \mathbb{D}$. Therefore

$$
\int|u|^{2}\left|\nabla w_{n}\right|^{2}=\int\left|\nabla w_{n}\right|^{2}+o(1) \quad \text { and } \quad \int|\nabla u|^{2}\left|w_{n}\right|^{2}=\int|\nabla u|^{2}+o(1) \quad \text { as } n \rightarrow \infty .
$$

Finally, since $\bar{u} w_{n} \nabla u \rightarrow \bar{u} \nabla u$ strongly in $L^{2}(\mathbb{D})$ and $\nabla w_{n} \rightarrow 0$ weakly in $L^{2}(\mathbb{D})$, we see that

$$
\int\left(u \nabla w_{n}\right) \cdot\left(w_{n} \nabla u\right)=o(1) \quad \text { as } n \rightarrow \infty
$$

This proves (8.15) and item 2 .

As for item 3., we start from the identity

$$
\int_{\mathbb{D}}\left(\left(u w_{n}\right) \wedge \nabla\left(u w_{n}\right)\right) \cdot \nabla \zeta=\int_{\mathbb{D}}\left(|u|^{2}-1\right)\left(w_{n} \wedge \nabla w_{n}\right) \cdot \nabla \zeta+\int_{\mathbb{D}}\left(w_{n} \wedge \nabla w_{n}\right) \cdot \nabla \zeta+\int_{\mathbb{D}}\left|w_{n}\right|^{2}(u \wedge \nabla u) \cdot \nabla \zeta,
$$

and argue as in the proof of Lemma 8.10.

A straightforward combination of the two preceding lemmas implies the main result of this section.

8.13 Theorem. Let $\left(v_{n}, \psi_{n}\right)$ be a Palais-Smale sequence associated to $J^{\sharp}$, and set $u_{n}^{*}:=v_{n}+u\left(N_{0} e^{l \psi_{n}}\right)$. Then, up to a subsequence, there exist: a critical point $u$ of $F_{\beta}$ in $\mathscr{G}$, an integer $K$, points $a_{1}(n), \ldots, a_{K}(n)$, corresponding bubbles $\mathscr{B}_{a_{j}(n)}, j \in \llbracket 1, K \rrbracket$ and a constant $\gamma \in \mathbb{S}^{1}$, such that

$$
\begin{aligned}
& \left|a_{j}(n)\right| \rightarrow 1 \text { as } n \rightarrow \infty, \forall j \in \llbracket 1, K \rrbracket ; \\
& u_{n}^{*}-\gamma u \prod_{j=1}^{K} \mathscr{B}_{a_{j}(n)} \rightarrow 0 \text { strongly in } H^{1}(\mathbb{D}) \text { as } n \rightarrow \infty \\
& F_{\beta}\left(u_{n}^{*}\right)=F_{\beta}(u)+K \pi+c_{n}, \quad \text { with } c_{n} \rightarrow 0 \text { as } n \rightarrow \infty
\end{aligned}
$$

In particular, we have (with $c^{\sharp}$ given by (8.5))

$$
c^{\sharp}=F_{\beta}(u)+K \pi .^{35}
$$

\footnotetext{
${ }^{35}$ Thus we have the following obvious upper bound on the number $K$ of bubbles: $K \leq c^{\sharp} / \pi$.
} 
Proof. From Lemma 8.12, we have $\left\|u_{n}^{*}-u w_{n}\right\|_{H^{1}} \rightarrow 0$ as $n \rightarrow \infty$, where $\left(w_{n}\right)$ satisfies the hypotheses of Lemma 8.10. In order to conclude, it suffices to apply Lemma 8.10 to $\left(w_{n}\right)$.

Results in the spirit of Theorem 8.13 has proven useful in many variational settings, especially in geometry. Let us simply mention the pioneering work of Sacks and Uhlenbeck [39] about minimal 2-spheres, the analysis of Brezis and Coron [17] of constant mean curvature surfaces, or the one of Struwe [41] of equations involving the critical Sobolev exponent. There are also abstract approaches to bubbling as in the work of Lions [33] about concentration-compactness or the characterization of lack of compactness of critical embeddings in Gérard [26], Jaffard [31] or Bahouri, Cohen and Koch [2].

We conclude with the following obvious consequence of our analysis of the Palais-Smale sequences.

8.14 Theorem. Assume that $c^{\sharp}$ given by (8.1) satisfies $c^{\sharp}<2 \pi$. Assume in addition that

there exists no solution $u \in \mathscr{G}$ of (2.23) such that $F_{\beta}(u)=c^{\sharp}-\pi$.

Then $F_{\beta}$ has a critical point $u \in \mathscr{G}_{1}$.

In addition, under the above assumptions the functional $F_{\beta}$ satisfies the $(P S)_{c}$ condition given by (5.6) at the level $c=c^{\sharp}$.

Note that, by Remark 8.1, our assumption on $c^{\sharp}$ generalizes assumption (6.1).

Theorem 8.14 combined with our next result implies Theorem 6.1 (and thus Theorem 1.1).

8.15 Lemma. Assume that (5.8) and (5.10) hold. Let $u$ be a critical point of $F_{\beta}$ in $\mathscr{G}_{\text {such that }}$ $F_{\beta}(u)<\pi$. Then $u$ is a constant (and thus $F_{\beta}(u)=0$ ).

Proof. Assumption (5.8) implies that critical points of $F_{\beta}$ are actually minimizers of $F_{\beta}$ with respect to their own boundary conditions. We next argue as in Step 1. in the proof of Lemma 8.10. If $u$ is such a minimizer and $F_{\beta}(u)<\pi$, then there exists some $a>0$ such that $|u| \geq a$ (Lemma 4.2 4.). Then we may write, globally in $\mathbb{D}, u=\rho e^{\imath \varphi}$ (Lemma 2.7 3.). Assume, in addition, that $u$ is a critical point of $F_{\beta}$. If we take $\zeta=\varphi$ in (2.21), then we find that $\varphi$ is constant, say $\varphi=0$. Thus $\rho$ satisfies $\left\{\begin{array}{ll}-\Delta \rho=\beta \rho\left(1-\rho^{2}\right) & \text { in } \mathbb{D} \\ \rho=1 & \text { on } \mathbb{S}^{1}\end{array}\right.$. By multiplying this equation with $\rho-1$, we find that $\rho \equiv 1$.

Open Problem 1. Let $u \in \mathscr{G}$ be a critical point of $F_{\beta}$. Assume that $F_{\beta}(u)<\pi .^{36}$ Is it true that $u$ is a constant? More generally, does the same hold if we replace the smallness assumption $F_{\beta}(u)<\pi$ by the weaker assumption $\frac{1}{2} \int_{\mathbb{D}}|\nabla u|^{2}<\pi ?$

\section{Bubbling analysis in multiply connected domains}

In this section, we establish the analog of Theorem 8.13 in multiply connected domains $\Omega$. To start with, this requires defining Palais-Smale sequences and bubbles. In defining Palais-Smale sequences, we can take as a starting point either Lemma 8.7 (and define a sequence $\left(u_{n}^{*}\right)$ ) or Lemma 8.8 (and define a sequence $\left(u_{n}\right)$ ). We adopt here the latter point of view.

\footnotetext{
${ }^{36}$ But we do not make any smallness assumption on $\beta$. In particular, we do not assume (5.8).
} 
9.1 Definition. Let $\beta \in L^{\infty}(\Omega)$. A sequence $\left(u_{n}\right) \subset \mathscr{E}$ is a Palais-Smale sequence for $F_{\beta}$ if there exists a sequence $c_{n} \rightarrow 0$ as $n \rightarrow \infty$ such that

$$
\begin{aligned}
& \left|\int_{\Omega} \nabla u_{n} \cdot \nabla w-\int_{\Omega} \beta u_{n} \cdot w\left(1-\left|u_{n}\right|^{2}\right)\right| \leq c_{n}\|\nabla w\|_{L^{2}}, \quad \forall w \in H_{0}^{1}(\Omega ; \mathbb{C}) ; \\
& \left|\int_{\Omega}\left(u_{n} \wedge \nabla u_{n}\right) \cdot \nabla \zeta\right| \leq c_{n}\|\nabla \zeta\|_{L^{2}}, \quad \forall \zeta \in H^{1}(\Omega ; \mathbb{R}) .
\end{aligned}
$$

We next define bubbles.

9.2 Definition. Let $\Gamma_{\ell}, \ell \in \llbracket 1, L \rrbracket$, be the components of $\partial \Omega$. We assume that $\Gamma_{1}$ encloses $\Omega$. Let $\omega_{1}$ be the simply connected domain enclosed by $\Gamma_{1}$, and for $\ell \geq 2$ let $\omega_{\ell}$ be the exterior domain bounded by $\Gamma_{\ell}$ in the extended plane $\mathbb{C} \cup\{\infty\}$. For each $\ell$, fix a conformal representation $\Phi_{\ell}: \omega_{\ell} \rightarrow \mathbb{D}$. For $a \in \mathbb{D}$ and $\ell \in \llbracket 1, L \rrbracket$, the corresponding bubble $\mathscr{B}_{a}^{\ell}$ is defined as either $\mathscr{B}_{a}^{\ell}=M_{1, a, \Phi_{\ell}}$, or $\mathscr{B}_{a}^{\ell}=\overline{M_{1, a, \Phi_{\ell}}}$.

Note that, unlike the case of simply connected domains, bubbles do not belong to $\mathscr{E}$. However, as $a \rightarrow \Gamma_{\ell}$, the trace of $\mathscr{B}_{a}^{\ell}$ almost fulfills the condition $\left|\operatorname{tr} \mathscr{B}_{a}^{\ell}\right|=1$.

The analog of Theorem 8.13 is

9.3 Theorem. Let $\left(u_{n}\right)$ be a Palais-Smale sequence. Then, up to a subsequence, there exist: a critical point $u$ of $F_{\beta}$ in $\mathscr{E}$, an integer $K$, indices $\ell_{1}, \ldots, \ell_{K} \in \llbracket 1, L \rrbracket$, points $a_{1}(n), \ldots, a_{K}(n) \in \Omega$, corresponding bubbles $\mathscr{B}_{a_{j}(n)}^{\ell_{j}}, j \in \llbracket 1, K \rrbracket$ and a constant $\gamma \in \mathbb{S}^{1}$, such that

$$
\begin{aligned}
& \operatorname{dist}\left(a_{j}(n), \Gamma_{l_{j}}\right) \rightarrow 0 \text { as } n \rightarrow \infty, \forall j \in \llbracket 1, K \rrbracket ; \\
& u_{n}-\gamma u \prod_{j=1}^{K} \mathscr{B}_{a_{j}(n)}^{l_{j}} \rightarrow 0 \text { strongly in } H^{1}(\Omega) \text { as } n \rightarrow \infty ; \\
& F_{\beta}\left(u_{n}\right)=F_{\beta}(u)+K \pi+c_{n}, \text { with } c_{n} \rightarrow 0 \text { as } n \rightarrow \infty .
\end{aligned}
$$

Proof. Up to a subsequence, we have $u_{n} \rightarrow u \in \mathscr{E}$ as $n \rightarrow \infty$. We define $g_{n}^{\ell}: \partial \Omega \rightarrow \mathbb{S}^{1}$ by

$$
g_{n}^{\ell}=\left\{\begin{array}{ll}
\operatorname{tr}\left(u_{n} / u\right) & \text { on } \Gamma_{\ell} \\
1 & \text { on } \partial \Omega \backslash \Gamma_{\ell}
\end{array},\right.
$$

so that $g_{n}:=\operatorname{tr}\left(u_{n} / u\right)$ satisfies $g_{n}=\prod_{\ell=1}^{L} g_{n}^{\ell}$. Let $v_{n}:=u\left(g_{n}\right)$ and $v_{n}^{\ell}:=u\left(g_{n}^{\ell}\right)$. An inspection of the proof of Lemma 8.12 shows that the conclusions of this lemma hold in our case. ${ }^{37}$ That is,

$$
u_{n}-v_{n} u \rightarrow 0 \text { strongly in } H^{1}(\Omega) \text {, and } F_{\beta}\left(u_{n}\right)=F_{\beta}(u)+\frac{1}{2} \int_{\Omega}\left|\nabla v_{n}\right|^{2}+c_{n} \text {, with } c_{n} \rightarrow 0 \text { as } n \rightarrow \infty \text {. }
$$

Moreover, the sequence $\left(v_{n}\right)$ satisfies (9.2).

We next prove an analogue of Lemma 8.10.

We first introduce two useful objects. Let $w_{n}^{\ell}$ denote the harmonic extension to $\omega_{\ell}$ of the trace of $g_{n}$ on $\Gamma_{\ell}$. We also set $W_{n}^{\ell}: \mathbb{D} \rightarrow \mathbb{C}, W_{n}^{\ell}:=w_{n}^{\ell} \circ\left(\Phi_{\ell}\right)^{-1}$.

\footnotetext{
${ }^{37}$ With $u_{n}$ instead of $u_{n}^{*}$.
} 
9.4 Lemma. We have

$$
\int_{\Omega}\left|\nabla v_{n}\right|^{2}=\sum_{\ell=1}^{L} \int_{\Omega}\left|\nabla w_{n}^{\ell}\right|^{2}+c_{n}, \quad \text { with } c_{n} \rightarrow 0 \text { as } n \rightarrow \infty
$$

and

$$
v_{n}-\prod_{\ell=1}^{L} w_{n}^{\ell} \rightarrow 0 \quad \text { strongly in } H^{1}(\Omega) \text { as } n \rightarrow \infty .
$$

Proof. By standard interior estimates [29, Proposition 1.13, p. 6] and global estimates [27, Theorem 9.19 , p. 243], the fact that $g_{n}^{\ell}-1$ in $H^{1 / 2}(\partial \Omega)$ as $n \rightarrow \infty$ and the fact that $g_{n}^{\ell}=1$ on $\partial \Omega \backslash \Gamma_{\ell}$ imply

$$
v_{n}^{\ell} \rightarrow 1 \quad \text { in } C_{l o c}^{k}\left(\bar{\Omega} \backslash \Gamma_{\ell}\right) \quad \text { as } n \rightarrow \infty .
$$

The same holds for $w_{n}^{\ell}$. It is then clear that $v_{n}-v_{n}^{\ell} \rightarrow 0$ in $C_{l o c}^{k}\left(\bar{\Omega} \backslash \cup_{j \neq \ell} \Gamma_{j}\right)$ as $n \rightarrow \infty$. Combined with (9.9), this fact leads to the expansion

$$
\int_{\Omega}\left|\nabla v_{n}\right|^{2}=\sum_{\ell=1}^{L} \int_{\Omega}\left|\nabla v_{n}^{\ell}\right|^{2}+c_{n}, \quad \text { with } c_{n} \rightarrow 0 \text { as } n \rightarrow \infty
$$

and to the strong convergence

$$
v_{n}-\prod_{\ell=1}^{L} v_{n}^{\ell} \rightarrow 0 \quad \text { in } C^{\infty}(\bar{\Omega}) \quad \text { as } n \rightarrow \infty .
$$

By the above, in order to conclude it suffices to invoke the fact that $\left|w_{n}^{\ell}\right| \leq 1$ (Lemma 2.17) and to prove that

$$
w_{n}^{\ell}-v_{n}^{\ell} \rightarrow 0 \quad \text { strongly in } H^{1}(\Omega) \quad \text { as } n \rightarrow \infty .
$$

In turn, (9.11) is obtained by noting that $y_{n}^{\ell}:=w_{n}^{\ell}-v_{n}^{\ell}$ is harmonic and that $\operatorname{tr} y_{n}^{\ell} \rightarrow 0$ in $H^{1 / 2}(\partial \Omega)$ as $n \rightarrow$ $\infty$.

9.5 Lemma. Up to a subsequence, the sequence $\left(W_{n}^{\ell}\right)_{n}$ satisfies the assumptions of Lemma 8.10.

As a consequence, up to a subsequence there are integers $K(\ell)$, points $a_{1}(n), \ldots, a_{K(\ell)}(n) \in \Omega$ and a constant $\gamma \in \mathbb{S}^{1}$ such that:

1. $\operatorname{dist}\left(a_{j}(n), \Gamma_{\ell}\right) \rightarrow 0$ as $n \rightarrow \infty, \forall j \in \llbracket 1, K(\ell) \rrbracket$.

2. $\frac{1}{2} \int_{\Omega}\left|\nabla w_{n}^{\ell}\right|^{2} \rightarrow K(\ell) \pi$ as $n \rightarrow \infty$.

3. $w_{n}^{\ell}-\gamma \prod_{j=1}^{K(\ell)} \mathscr{B}_{a_{j}(n)}^{\ell} \rightarrow 0$ strongly in $H^{1}(\Omega)$ as $n \rightarrow \infty$.

Proof. We have to prove that the sequence $\left(W_{n}^{\ell}\right)_{n}$ satisfies (8.9). By conformal invariance, it suffices to check the same property for $\left(w_{n}^{\ell}\right)_{n}$. By (9.11), we are reduced to checking the same for $\left(v_{n}^{\ell}\right)_{n}$. Let $\Omega_{\ell} \subset \mathbb{C}$ be an open set such that $\Omega_{\ell} \cap \partial \Omega=\Gamma_{\ell}$. Let $\eta_{\ell} \in C_{c}^{\infty}\left(\Omega_{\ell} ; \mathbb{R}\right)$ be such that $\eta_{\ell}=1$ in a neighborhood 
of $\Gamma_{\ell}$. If $\zeta \in H^{1}(\Omega ; \mathbb{R})$, set $\xi_{\ell}:=\eta_{\ell} \zeta, \lambda_{\ell}:=\zeta-\xi_{\ell}$. Assuming in addition that $\int_{\Omega} \zeta=0$, we have the Poincaré type inequality

$$
\left\|\nabla \xi_{\ell}\right\|_{L^{2}}+\left\|\nabla \lambda_{\ell}\right\|_{L^{2}} \leq C\|\nabla \zeta\|_{L^{2}} \cdot{ }^{38}
$$

Using Lemma 8.12, (9.9) and (9.10), we find that

$$
\begin{aligned}
\left|\int_{\Omega}\left(v_{n}^{\ell} \wedge \nabla v_{n}^{\ell}\right) \cdot \nabla \xi_{\ell}\right| & \leq\left|\int_{\Omega_{\ell}}\left(v_{n}^{\ell} \wedge \nabla v_{n}^{\ell}\right) \cdot \nabla \xi_{\ell}\right|+\left|\int_{\Omega \backslash \Omega_{\ell}}\left(v_{n}^{\ell} \wedge \nabla v_{n}^{\ell}\right) \cdot \nabla \xi_{\ell}\right| \\
& \leq\left|\int_{\Omega_{\ell}}\left(v_{n} \wedge \nabla v_{n}\right) \cdot \nabla \xi_{\ell}\right|+c_{n} \int_{\Omega}\left|\nabla \xi_{\ell}\right| \leq c_{n}\left\|\nabla \xi_{\ell}\right\|_{L^{2}}, \quad \text { with } c_{n} \rightarrow 0 \text { as } n \rightarrow \infty .
\end{aligned}
$$

Similarly, we have

$$
\left|\int_{\Omega}\left(v_{n}^{\ell} \wedge \nabla v_{n}^{\ell}\right) \cdot \nabla \lambda_{\ell}\right| \leq c_{n}\left\|\nabla \lambda_{\ell}\right\|_{L^{2}}, \quad \text { with } c_{n} \rightarrow 0 \text { as } n \rightarrow \infty .
$$

We obtain (8.9) for the sequence $\left(v_{n}^{\ell}\right)$ by combining (9.12)-(9.14).

Items 1. and 3. are obtained from the analog results for $W_{n}^{\ell}$ via composition with $\Phi_{\ell}$. As for item $2 .$, it follows from (8.8) once we note that

$$
\int_{\Omega}\left|\nabla w_{n}^{\ell}\right|^{2}=\int_{\mathbb{D}}\left|\nabla W_{n}^{\ell}\right|^{2}-\int_{\mathbb{D} \backslash\left(\Phi_{\ell}\right)^{-1}(\Omega)}\left|\nabla W_{n}^{\ell}\right|^{2}=\int_{\mathbb{D}}\left|\nabla W_{n}^{\ell}\right|^{2}+c_{n}, \quad \text { with } c_{n} \rightarrow 0 \text { as } n \rightarrow \infty
$$

here, we use the fact that $W_{n}^{\ell} \rightarrow 1$ in $C_{l o c}^{k}(\mathbb{D})$ as $n \rightarrow \infty$.

Proof of Theorem 9.3 completed. By combining Lemma 9.4 with Lemma 9.5, we obtain an analog of Lemma 8.10, i.e.,

$$
v_{n}-\gamma \prod_{j=1}^{K} \mathscr{B}_{a_{j}(n)}^{\ell_{j}} \rightarrow 0 \text { strongly in } H^{1}(\Omega) \text { as } n \rightarrow \infty, \text { and } \frac{1}{2} \int_{\Omega}\left|\nabla v_{n}\right| \rightarrow K \pi \text { as } n \rightarrow \infty,
$$

for some integer $K$, for some constant $\gamma \in \mathbb{S}^{1}$ and for some points $a_{1}(n), \ldots, a_{K}(n) \in \Omega$ satisfying (9.3). Using this fact in (9.6), we obtain (9.4) and (9.5).

We conclude with an application of the above analysis to the existence of critical points in doubly connected domains. For simplicity, we assume that we have circular symmetry and we let $\Omega=\mathbb{D} \backslash \overline{\mathbb{D}}_{R}$, with $0<R<1$, but Theorem 9.6 below extends to arbitrary doubly connected domains. With $\mathbf{d}=$ $\left(d_{1}, d_{2}\right) \in \mathbb{Z}^{2}$, we let

$$
\mathscr{E}_{\mathbf{d}}:=\left\{u \in \mathscr{E} ; \operatorname{deg}\left(u, \mathbb{S}^{1}\right)=d_{1}, \operatorname{deg}\left(u, C_{R}\right)=d_{2}\right\} .
$$

Our result is the following.

9.6 Theorem. Assume that $R$ is sufficiently small. Then there exists some $\varepsilon_{0}$ such that, for $\varepsilon \in\left(\varepsilon_{0}, \infty\right]$, $E_{\varepsilon}$ has critical points in $\mathscr{E}_{(1,0)}$.

The above result is reminiscent of Coron's result on the existence of non trivial solution of the equation $-\Delta u=u^{(n+2) /(n-2)}$ in domains $\Omega \subset \mathbb{R}^{n}$ with small holes [22]. Since later Bahri and Coron [3] proved that the size of the hole is irrelevant for existence of such solutions, we address the following Open Problem 2. Let $R \in(0,1)$. Does there exist some $\varepsilon_{0}>0$ such that $E_{\varepsilon}$ has critical points in $\mathscr{E}_{(1,0)}$ for $\varepsilon \in\left(\varepsilon_{0}, \infty\right]$ ?

\footnotetext{
${ }^{38}$ This is easily obtained by contradiction.
} 
Proof of Theorem 9.6. We start by describing the functional setting. We let, with $0<r<1$,

$$
K=\overline{\mathbb{D}}_{r}, K_{0}=C_{r}, X^{\sharp}=H_{0}^{1}(\Omega ; \mathbb{C}) \times H^{1 / 2}(\partial \Omega ; \mathbb{R}) .
$$

Let $N: \partial \Omega \rightarrow \mathbb{S}^{1}, N(z)=\left\{\begin{array}{ll}z, & \text { if }|z|=1 \\ 1, & \text { if }|z|=R\end{array}\right.$. Then we define

$$
J^{\sharp}: X^{\sharp} \rightarrow \mathbb{R}, \quad H_{0}^{1}(\Omega ; \mathbb{C}) \times H^{1 / 2}(\partial \Omega ; \mathbb{R}) \ni(v, \psi) \stackrel{J^{\sharp}}{\longrightarrow} E_{\varepsilon}\left(v+u\left(N e^{\imath \psi}\right)\right) .
$$

Note that $v+u\left(N e^{\imath \psi}\right) \in \mathscr{E}_{(1,0)}$. For $a \in \mathbb{D}$, we write, as in Section $5, N_{a}=N_{0} e^{\imath \psi_{a}}$, and let $\eta_{a}=$ $\left\{\begin{array}{ll}\psi_{a}, & \text { on } \mathbb{S}^{1} \\ 0, & \text { on } C_{R}\end{array}\right.$. We then set

$$
\chi^{\sharp} \in C\left(K_{0} ; X^{\sharp}\right), \quad K_{0} \ni a \stackrel{\chi^{\sharp}}{\rightarrow}\left(0, \eta_{a}\right)
$$

and define

$$
c=c_{R, r, \varepsilon}=\inf \left\{\max _{K} J^{\sharp} \circ F ; F \in C\left(K ; X^{\sharp}\right), F=\chi^{\sharp} \text { on } K_{0}\right\} .
$$

The plan is to prove that, for small $R$ and large $\varepsilon$, the compactness condition (PS) $c$ described in (5.6) is satisfied. This will imply existence of critical points of $E_{\varepsilon}$ in $\mathscr{E}_{(1,0)}$.

To start with, note that, as in the proof of Lemma 3.1, we have

$$
E_{\varepsilon}(u) \geq \frac{1}{2} \int_{\Omega}|\nabla u|^{2} \geq \int_{\Omega}|\operatorname{Jac} u| \geq\left|\int_{\Omega} \operatorname{Jac} u\right|=\pi, \quad \forall u \in \mathscr{E}_{(1,0)} .
$$

Before proceeding further, we establish few auxiliary results.

9.7 Lemma. Let $\delta>0$. Then there exist $R_{0}<1, r_{0}<1$, and $\varepsilon_{0}>0$ such that $c=c_{R, r, \varepsilon}<\pi+\delta$ provided $R<R_{0}, r>r_{0}$ and $\varepsilon>\varepsilon_{0}$.

We also have

$$
\lim _{r \rightarrow 1} \max _{K_{0}} J^{\sharp} \circ \chi^{\sharp}=\pi \text {. }
$$

Proof. Let $\delta^{\prime}>0$ to be fixed later. Pick $R_{0}$ such that for $R<R_{0}$ there exists some $\zeta_{R} \in C^{\infty}(\bar{\Omega} ;[0,1])$ such that $\zeta_{R}=1$ near $\mathbb{S}^{1}, \zeta_{R}=0$ near $C_{R}$ and $\int_{\Omega}\left|\nabla \zeta_{R}\right|^{2}<\delta^{\prime}$. This is always possible provided $R_{0}$ is sufficiently small, since the $H^{1}$-capacity of a point is zero. Define

$$
P: \mathbb{D} \rightarrow \mathscr{E}_{(1,0)}, \quad D \ni a \stackrel{P}{\rightarrow} \zeta_{R} M_{a}+1-\zeta_{R} .
$$

Let also $F(a)=\left(0, \eta_{a}\right), a \in K$. Then $\operatorname{tr} P(a)=\operatorname{tr} u\left(N e^{\imath \eta_{a}}\right)$, and a straightforward calculation leads to

$$
\begin{aligned}
c \leq \max _{K} J^{\sharp} \circ F & =\max _{a \in K} E_{\varepsilon}\left(u\left(N e^{\imath \eta_{a}}\right)\right) \leq \max _{a \in K} E_{\varepsilon}(P(a)) \\
& \leq \max _{a \in K}\left(\frac{1}{2} \int_{\Omega}\left(1-\zeta_{R}\right)^{2}\left|\nabla M_{a}\right|^{2}+C\left(\delta^{\prime}+\sqrt{\delta^{\prime}}+\varepsilon^{-2}\right)\right) \leq \pi+C\left(\delta^{\prime}+\sqrt{\delta^{\prime}}+\varepsilon^{-2}\right) .
\end{aligned}
$$

This implies the first part of the lemma. For the second part, let us note that, when $|a| \rightarrow 1$, we have $\bar{a} M_{a} \rightarrow-1$ in $C_{l o c}^{k}(\mathbb{D})$, and this leads to

$$
\limsup _{|a| \rightarrow 1} E_{\varepsilon}(P(a)) \leq \pi .
$$

We conclude by combining (9.17) with (9.15). 
The next result is the content of [5, Lemma D.3].

9.8 Lemma. We have

$$
\inf \left\{E_{\varepsilon}(u) ; u \in \mathscr{E}_{(1,1)}\right\} \geq \min \left\{E_{\infty}(u) ; u \in \mathscr{E}_{(1,1)}\right\}=2 \pi \frac{1-R}{1+R} .
$$

\subsection{Lemma. Consider the minimization problem}

$$
m_{\varepsilon}=\inf \left\{E_{\varepsilon}(u) ; u \in \mathscr{E}_{(1,0)}\right\}
$$

Then

1. $m_{\varepsilon}=\pi$ and $m_{\varepsilon}$ is not attained.

2. If $\left(u_{n}\right)$ is a minimizing sequence in (9.19), then, up to a subsequence, $u_{n} \rightarrow \alpha \in \mathbb{S}^{1}$ in $H^{1}(\Omega)$.

Proof. Let $\Phi_{t}: \mathbb{C} \rightarrow \mathbb{C}, \Phi_{t}(z)=\left\{\begin{array}{ll}z / t, & \text { if }|z| \leq t \\ z /|z| & \text { if }|z| \geq t\end{array}\right.$. It is easy to see that, when $a \in \mathbb{D}$ and $|a| \rightarrow 1$, we may pick some $t=t(a) \rightarrow 1$ such that we have $\Phi_{t} \circ M_{a} \in \mathscr{E}_{(1,0)}$ and $E_{\varepsilon}\left(\Phi_{t} \circ M_{a}\right) \rightarrow \pi$. This together with (9.15) implies that $m_{\varepsilon}=\pi$. As in the proof of Lemma 3.1, if $E_{\varepsilon}(u)=\pi$, then $u$ is holomorphic. However, we claim that there is no holomorphic map in $\mathscr{E}_{(1,0)}$. Indeed, otherwise we have:

a) $u(\Omega) \subset \mathbb{D}$ (by the maximum principle).

b) $u(\Omega) \supset \mathbb{D}$ (since the total degree of $u$ on $\partial \Omega$ is one).

c) $\int_{\Omega}|\operatorname{Jac} u|=\pi$.

By the above and the area formula, for a.e. $a \in \mathbb{D}$ the set $u^{-1}(a)$ is a singleton. The latter fact combined with the open mapping theorem for holomorphic functions implies that $u$ is one-to-one. In conclusion, $u: \Omega \rightarrow \mathbb{D}$ is a homeomorphism, which is the desired contradiction (since $\Omega$ and $\mathbb{D}$ are not homeomorphic).

Consider now a minimizing sequence $\left(u_{n}\right)$ and assume that $u_{n} \rightarrow u$ as $n \rightarrow \infty$. Since $u \notin \mathscr{E}_{(1,0)}$, we find that $u$ is a constant, by the Price Lemma 2.18. This constant has to be of modulus one.

The next result is the analog of Theorem 4.1 in doubly connected domains. If $u \in H^{1}(\Omega)$, then we define $\underline{u}$ as the harmonic extension to $\mathbb{D}$ of the trace of $u$ on $\mathbb{S}^{1}$. In particular, if $u \in \mathscr{E}_{(1,0)}$, then $\underline{u} \in \mathscr{E}_{1}$.

9.10 Lemma. There exists some $\delta_{0}=\delta_{0}(R)>0$ and a function $f:\left(0, \delta_{0}\right) \rightarrow(0, \infty)$ such that $\lim _{\delta \rightarrow 0} f(\delta)=0$ with the following property: if $u \in \mathscr{E}_{(1,0)}$ satisfies $\frac{1}{2} \int_{\Omega}|\nabla u|^{2}<\pi+\delta$, with $\delta<\delta_{0}$, then $\frac{1}{2} \int_{\mathbb{D}}^{\delta \rightarrow 0}|\nabla|^{2}<$ $\pi+f(\delta)$.

In particular, if $\delta_{0}$ is sufficiently small then there exists a continuous map

$$
H:\left\{u \in \mathscr{E}_{(1,0)} ; \frac{1}{2} \int_{\Omega}|\nabla u|^{2}<\pi+\delta_{0}\right\} \rightarrow \mathbb{D}, \quad u \stackrel{H}{\rightarrow} a(\underline{u})
$$

where $a(\underline{u})$ is the unique zero of $\underline{u}$.

Moreover, we have

$$
|H(u)| \rightarrow 1 \text { when } \frac{1}{2} \int_{\mathbb{D}}|\nabla u|^{2} \rightarrow \pi .
$$


Proof. For the first part of the lemma, it suffices to prove that, if $\left(u_{n}\right) \subset \mathscr{E}_{(1,0)}$ and $\frac{1}{2} \int_{\Omega}\left|\nabla u_{n}\right|^{2} \rightarrow \pi$ as $n \rightarrow \infty$, then (possibly along a subsequence) $\frac{1}{2} \int_{\mathbb{D}}\left|\nabla \underline{u_{n}}\right|^{2} \rightarrow \pi$. By (9.15), we may assume that $u_{n}$ is harmonic. By Lemma 9.9 2., we have, up to a subsequence, $u_{n} \rightarrow \alpha$ in $C_{l o c}^{k}(\Omega)$. Thus, for $R<r<1$, the restriction of $u_{n}$ to $\mathbb{D} \backslash \overline{\mathbb{D}}_{r}$ has an extension $v_{n}$ to $\mathbb{D}$ such that

$$
\frac{1}{2} \int_{\mathbb{D}}\left|\nabla \underline{u_{n}}\right|^{2} \leq \frac{1}{2} \int_{\mathbb{D}}\left|\nabla v_{n}\right|^{2} \leq \frac{1}{2} \int_{\Omega}\left|\nabla u_{n}\right|^{2}+c_{n} \quad \text { with } c_{n} \rightarrow 0 \text { as } n \rightarrow \infty .
$$

The second part of the theorem follows from Theorem 4.1. As for the last part, consider a sequence $\left(u_{n}\right) \subset \mathscr{E}_{(1,0)}$ such that $\frac{1}{2} \int_{\Omega}\left|\nabla u_{n}\right|^{2} \rightarrow \pi$. By Lemma 9.9, we have, up to a subsequence, $u_{n} \rightarrow \alpha \in \mathbb{S}^{1}$, and thus $\underline{u_{n}} \rightarrow \alpha$, whence the conclusion.

Proof of Theorem 9.6 completed. We start by proving that $c>\pi$. Indeed, let $\delta_{0}=\delta_{0}(R)$ be such that $H$ given by (9.20) is well-defined and satisfies $|H(u)| \geq 1 / 2$. We claim that $c \geq \pi+\delta_{0}$. Indeed, otherwise let $F \in C\left(K ; X^{\sharp}\right)$ be such that $F=\chi^{\sharp}$ on $K_{0}$ and $J^{\sharp} \circ F<\pi+\delta_{0}$. Let

$$
G: \overline{\mathbb{D}}_{r} \rightarrow \mathbb{D} \backslash \mathbb{D}_{1 / 2}, \quad G:=H \circ U^{\sharp} \circ F .
$$

Here,

$$
U^{\sharp}: X^{\sharp} \rightarrow \mathscr{E}_{(1,0)}, \quad X^{\sharp} \ni(v, \psi) \stackrel{U^{\sharp}}{\longrightarrow} v+u\left(N e^{l \psi}\right) .
$$

By construction, $G$ is continuous and we have $G=\operatorname{Id}$ on $C_{r}$. This contradicts Brouwer's fixed point theorem.

The fact that $c>\pi$ combined with (9.16) implies that for $r$ close to 1 we have

$$
c>\max _{K_{0}} J^{\sharp} \circ \chi^{\sharp},
$$

and that $c$ does not depend on $r$ close to 1 .

(9.22), combined with the proofs of Lemmas 8.7 and 8.8 and with Lemma 9.7 and Theorem 9.3 leads to the following: if $R<R_{0}, r>r_{0}$ and $\varepsilon>\varepsilon_{0}$, then:

1. $\pi<c<\min \left\{2 \pi, \pi+2 \pi \frac{1-R}{1+R}\right\}$.

2. There exists a sequence $\left(u_{n}\right) \subset \mathscr{E}_{(1,0)}$ as in Theorem $9.3 .^{39}$

We complete the proof of Theorem 9.6 if we prove that the integer $K$ in Theorem 9.3 is zero. Let $u$ be as in Theorem 9.3, and let $\mathbf{D} \in \mathbb{Z}^{2}$ be such that $u \in \mathscr{E}_{\mathbf{D}}$. By Theorem 9.3 and the fact that $c<2 \pi$, one of the following cases occurs:

1. $K=0$ and $\mathbf{D}=(1,0)$, which is the desired conclusion.

2. $K=1, \mathbf{D}=(1,1)$ and $E_{\varepsilon}(u)=c-\pi$.

3. $K=1, \mathbf{D}=(0,0)$ and $E_{\varepsilon}(u)=c-\pi$.

\footnotetext{
${ }^{39}$ With $E_{\varepsilon}$ instead of $F_{\beta}$.
} 
The second case is ruled out thanks to Lemma 9.8 and to the fact that $c-\pi<2 \pi \frac{1-R}{1+R}$.

We next turn to the third case. Since $\frac{1}{2} \int_{\Omega}|\nabla u|^{2}<\pi,{ }^{40}$ Lemma 4.24 . implies that $|u| \geq a>0$ in $\Omega$. By Lemma 2.8, in $\Omega$ we may globally write $u=\rho e^{\imath \varphi}$. As in the proof of Lemma 8.15, this implies that $u$ is constant. This contradicts the fact that $c>\pi$.

The proof of Theorem 9.6 is complete.

\section{References}

[1] A. Ambrosetti and P.H. Rabinowitz. Dual variational methods in critical point theory and applications. J. Funct. Anal., 14:349-381, 1973.

[2] H. Bahouri, A. Cohen, and G. Koch. A general wavelet-based profile decomposition in the critical embedding of function spaces. Confluentes Math., 3(3):387-411, 2011.

[3] A. Bahri and J.-M. Coron. On a nonlinear elliptic equation involving the critical Sobolev exponent: the effect of the topology of the domain. Comm. Pure Appl. Math., 41(3):253-294, 1988.

[4] L.V. Berlyand, D. Golovaty, and V. Rybalko. Nonexistence of Ginzburg-Landau minimizers with prescribed degree on the boundary of a doubly connected domain. C. R. Math. Acad. Sci. Paris, 343(1):63-68, 2006.

[5] L.V. Berlyand and P. Mironescu. Ginzburg-Landau minimizers in perforated domains with prescribed degrees. http://math.univ-lyon1.fr/ mironescu/3.pdf.

[6] L.V. Berlyand and P. Mironescu. Ginzburg-Landau minimizers with prescribed degrees. Capacity of the domain and emergence of vortices. J. Funct. Anal., 239(1):76-99, 2006.

[7] L.V. Berlyand, O. Misiats, and V. Rybalko. Near boundary vortices in a magnetic GinzburgLandau model: their locations via tight energy bounds. J. Funct. Anal., 258(5):1728-1762, 2010.

[8] L.V. Berlyand, O. Misiats, and V. Rybalko. Minimizers of the magnetic Ginzburg-Landau functional in simply connected domain with prescribed degree on the boundary. Commun. Contemp. Math., 13(1):53-66, 2011.

[9] L.V. Berlyand and V. Rybalko. Solutions with vortices of a semi-stiff boundary value problem for the Ginzburg-Landau equation. J. Eur. Math. Soc. (JEMS), 12(6):1497-1531, 2010.

[10] L.V. Berlyand and K. Voss. Symmetry Breaking in Annular Domains for a Ginzburg-Landau Superconductivity Model. Proceedings of IUTAM 99/4 Symposium, Sydney, Australia. Kluwer Academic Publishers, 2001.

[11] F. Bethuel, H. Brezis, and F. Hélein. Ginzburg-Landau vortices. Progress in Nonlinear Differential Equations and their Applications, 13. Birkhäuser Boston Inc., Boston, MA, 1994.

[12] F. Bethuel and J.-M. Ghidaglia. Improved regularity of solutions to elliptic equations involving Jacobians and applications. J. Math. Pures Appl. (9), 72(5):441-474, 1993.

\footnotetext{
${ }^{40}$ Thanks to the condition $c<2 \pi$.
} 
[13] G. Bourdaud and D. Kateb. Fonctions qui opérent sur les espaces de Besov. Proc. Amer. Math. Soc., 112(4):1067-1076, 1991.

[14] J. Bourgain, H. Brezis, and P. Mironescu. Lifting in Sobolev spaces. J. Anal. Math., 80:37-86, 2000 .

[15] A. Boutet de Monvel-Berthier, V. Georgescu, and R. Purice. A boundary value problem related to the Ginzburg-Landau model. Comm. Math. Phys., 142(1):1-23, 1991.

[16] H. Brezis. Critical points in variational problems without compactness. Astérisque, 1987/88(161162):239-256, 1988.

[17] H. Brezis and J.-M. Coron. Convergence of solutions of $H$-systems or how to blow bubbles. Arch. Rational Mech. Anal., 89(1):21-56, 1985.

[18] H. Brezis, J.-M. Coron, and L. Nirenberg. Free vibrations for a nonlinear wave equation and a theorem of P. Rabinowitz. Comm. Pure Appl. Math., 33(5):667-684, 1980.

[19] H. Brezis and P. Mironescu. Sobolev Maps with Values into the Circle. Analytical, Geometrical and Topological Aspects. Birkhäuser Boston Inc., In preparation.

[20] H. Brezis and L. Nirenberg. Degree theory and BMO. I. Compact manifolds without boundaries. Selecta Math. (N.S.), 1(2):197-263, 1995.

[21] H. Brezis and L. Nirenberg. Degree Theory and BMO. II. Compact manifolds with boundaries. Selecta Math. (N.S.), 2:309-368, 1996.

[22] J.-M. Coron. Topologie et cas limite des injections de Sobolev. C. R. Acad. Sci. Paris Sér. I Math., 299(7):209-212, 1984.

[23] M. Dos Santos. Local minimizers of the Ginzburg-Landau functional with prescribed degrees. J. Funct. Anal., 257(4):1053-1091, 2009.

[24] A. Farina and P. Mironescu. Uniqueness of vortexless Ginzburg-Landau type minimizers in two dimensions. Calc. Var. Partial Differential Equations, 46(3-4):523-554, 2013.

[25] E. Gagliardo. Caratterizzazioni delle tracce sulla frontiera relative ad alcune classi di funzioni in $n$ variabili. Rend. Sem. Mat. Univ. Padova, 27:284-305, 1957.

[26] P. Gérard. Description du défaut de compacité de l'injection de Sobolev. ESAIM Control Optim. Calc. Var., 3:213-233 (electronic), 1998.

[27] D. Gilbarg and N.S. Trudinger. Elliptic partial differential equations of second order. Classics in Mathematics. Springer-Verlag, Berlin, 2001. Reprint of the 1998 edition.

[28] D. Golovaty and L.V. Berlyand. On uniqueness of vector-valued minimizers of the ginzburglandau functional in annular domains. Calc. Var. Partial Differential Equations, 14(2):213-232, 2002.

[29] Q. Han and F.H. Lin. Elliptic partial differential equations, volume 1 of Courant Lecture Notes in Mathematics. New York University Courant Institute of Mathematical Sciences, New York, 1997. 
[30] F. Hélein. Constant mean curvature surfaces, harmonic maps and integrable systems. Lectures in Mathematics ETH Zürich. Birkhäuser Verlag, Basel, 2001. Notes taken by Roger Moser.

[31] S. Jaffard. Analysis of the lack of compactness in the critical Sobolev embeddings. J. Funct. Anal., 161(2):384-396, 1999.

[32] X. Lamy and P. Mironescu. Existence of critical points with semi-stiff boundary conditions for singular perturbation problems in simply connected planar domains. http:/hal.archivesouvertes.fr/hal-00784904, Feb 2013. 47 p.

[33] P.-L. Lions. The concentration-compactness principle in the calculus of variations. The limit case. II. Rev. Mat. Iberoamericana, 1(2):45-121, 1985.

[34] J. Mawhin and M. Willem. Critical point theory and Hamiltonian systems, volume 74 of Applied Mathematical Sciences. Springer-Verlag, New York, 1989.

[35] V. Millot and Y. Sire. On a fractional Ginzburg-Landau system and 1/2-harmonic maps into spheres. In preparation.

[36] F. Pacard and T. Rivière. Linear and nonlinear aspects of vortices. Progress in Nonlinear Differential Equations and their Applications, 39. Birkhäuser Boston Inc., Boston, MA, 2000. The Ginzburg-Landau model.

[37] A.C. Ponce. Équation de Ginzburg-Landau et singularités. Lecture notes of the graduate course of H. Brezis. 78 p. http://perso.uclouvain.be/augusto.ponce/links/04-cours-gl.pdf., 2004.

[38] T. Runst and W. Sickel. Sobolev spaces of fractional order, Nemytskij operators, and nonlinear partial differential equations, volume 3 of de Gruyter Series in Nonlinear Analysis and Applications. Walter de Gruyter \& Co., Berlin, 1996.

[39] J. Sacks and K. Uhlenbeck. The existence of minimal immersions of 2-spheres. Ann. of Math. (2), 113(1):1-24, 1981.

[40] S. Serfaty. Stability in 2d Ginzburg-Landau passes to the limit. Indiana Univ. Math. J., 54(1):199-221, 2005.

[41] M. Struwe. A global compactness result for elliptic boundary value problems involving limiting nonlinearities. Math. Z., 187(4):511-517, 1984.

[42] H.C. Wente. An existence theorem for surfaces of constant mean curvature. J. Math. Anal. Appl., 26:318-344, 1969.

[43] B. White. Homotopy classes in Sobolev spaces and the existence of energy minimizing maps. Acta Math., 160(1-2):1-17, 1988. 